\title{
Dietary sources of omega 3 fatty acids: public health risks and benefits
}

\author{
J. A. Tur*, M. M. Bibiloni, A. Sureda and A. Pons \\ Research Group on Community Nutrition and Oxidative Stress, Universitat de les Illes Balears, Guillem Colom Bldg Campus, \\ E-07122 Palma de Mallorca, Spain
}

\section{Abstract}

Omega 3 fatty acids can be obtained from several sources, and should be added to the daily diet to enjoy a good health and to prevent many diseases. Worldwide, general population use omega-3 fatty acid supplements and enriched foods to get and maintain adequate amounts of these fatty acids. The aim of this paper was to review main scientific evidence regarding the public health risks and benefits of the dietary sources of omega-3 fatty acids. A systematic literature search was performed, and one hundred and forty-five articles were included in the results for their methodological quality. The literature described benefits and risks of algal, fish oil, plant, enriched dairy products, animal-derived food, krill oil, and seal oil omega-3 fatty acids.

Key words: Omega 3: Public health: Health risks: Health benefits: Systematic review

Omega 3 fatty acids can be obtained from several sources, and should be added to the daily diet to enjoy a good health and to prevent many diseases. The European Food Safety Agency (EFSA) proposed a recommended daily intake of $250 \mathrm{mg} / \mathrm{d}$ eicosapentaenoic acid (EPA) and docosahexaenoic acid (DHA) for adults, because this intake is negatively related to cardiovascular diseases (CVD) risk in a dose-dependent way up to $250 \mathrm{mg} / \mathrm{d}$ (1-2 servings/week of oily fish) in healthy subjects $^{(1)}$. The American Heart Association (AHA) recommended for the general population a consumption of fish, at least twice a week ${ }^{(2)}$, estimating a consumption of one portion ( $125 \mathrm{~g}$ ) of oily fish ( $2 \mathrm{~g} / 100 \mathrm{~g}$ EPA and DHA) and one portion of lean fish $(0 \cdot 2 \mathrm{~g} / 100 \mathrm{~g})$, which results in an mean intake of $3 \mathrm{~g} /$ week or $430 \mathrm{mg} / \mathrm{d}$ of DHA and EPA. AHA also established intakes of $1 \mathrm{~g}$ of EPA and DHA from fish or fish oils for subjects with clinical history of CVD and a $2-4 \mathrm{~g}$ supplement for subjects with high blood triacylglycerides $(\mathrm{TAG})^{(3)}$. The World Health Organization (WHO) recommended a regular fish consumption (1-2 servings/week; providing $200-500 \mathrm{mg} /$ serving of EPA and DHA) for the general population, as being protective against coronary heart disease and ischemic stroke $\mathrm{e}^{(4)}$. WHO also indicates that vegetarians and not fish-eaters are recommended to ensure adequate intake of plant sources of alpha-linolenic acid (ALA), as some of it (0.5-20\% depending on various factors) is metabolized to $\mathrm{EPA}^{(5,6)}$. Worldwide, general population use omega-3 fatty acids supplements and enriched foods to get and maintain adequate amounts of these fatty acids, i.e.: milk and dairy products are every day consumed foods and constitute a good and popular source of omega-3 fatty acids, to produce 'healthier' milks and dairy products ${ }^{(7)}$. The aim of this paper was to review main scientific evidence regarding the public health risks and benefits of the dietary sources of omega-3 fatty acids

\section{Methods}

A systematic literature search was performed up to April 2011. The literature search was conducted in Medlars Online International Literature (MEDLINE), via PubMed ${ }^{\circledR}$; Scopus; OvidSP (Food Science and Technology Abstracts); EMBA$\mathrm{SE}^{\odot}$, and Latin American and Caribbean Heath Sciences Literature (LILACS), using the following terms: 'Fatty acids, omega-3'[Major] OR 'alpha-linolenic acid'[Mesh] OR 'docosahexaenoic acids'[Mesh] OR 'eicosapentaenoic acid'[Mesh] AND ('adverse effects'[Mesh] OR 'contraindications'[Mesh] OR 'standards'[Mesh] OR 'supply and distribution'[Mesh] OR 'therapeutic use'[Mesh] OR 'toxicity'[Mesh] AND ('humans' [MeSH Terms] AND ('Clinical Trial'[ptyp] OR 'Randomized Controlled Trial'[ptyp])).

Using the above mentioned data bases, 2476 articles were selected. Duplicates, review articles and non-relevant articles were excluded ( $n$ 2310). After reading the literature list of the remaining articles, and suggestions from other experts about relevant papers, 35 were included in the results. Fiftyfour of the remaining 201 articles were rejected for the reasons shown in Fig. 1. Finally, just one hundred and forty-seven articles were included in the results. The articles were reviewed by at least two reviewers and were taken into account for the selection criteria listed on the JADAD scale 


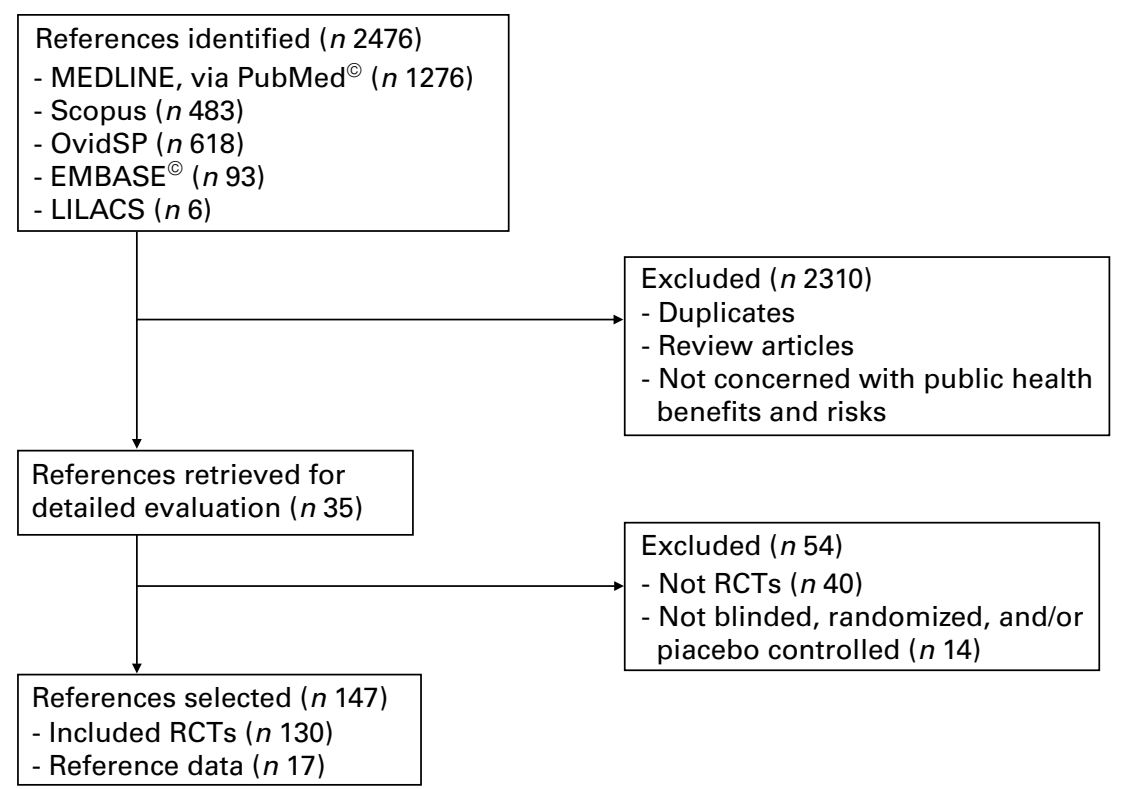

Fig. 1. Literature search flow chart.

or Oxford Quality Scoring System, a procedure to independently assess the methodological quality of a clinical trial. Reviewers extracted data from the published reports. Table 1 summarises the design of and the results provided by the 147 articles finally selected.

\section{Results \\ Algal omega-3 fatty acids}

Clinical trials with algal DHA-rich oil supplementation resulted in potentially beneficial changes in some markers of cardiometabolic risk: decrease in VLDL and increase in LDL and HDL particle sizes, and reduction in VLDL, and total TAG concentrations, blood pressure and heart rate, and oxidative stress, indicating comparable efficacies to fish oil. Algal-DHA was safe and well tolerated ${ }^{(8)}$. Unlike fish oil, algal-DHA seldom caused gastrointestinal complaints such as fishy taste and eructation, attributes of importance for patient compliance in high-dose therapy.

The consumption of Ulkenia sp. microalgae oil (0.94 g DHA/d) for 8 weeks decreased plasma TAG, and increased plasma total cholesterol, LDL- and HDL-cholesterol in normolipidaemic vegetarians. DHA-rich oil from Ulkenia sp. is well tolerated and a suitable vegetarian source of $n$-3 LC-PUFAs ${ }^{(9)}$.

Schizochytrium sp. microalgae provided substantial quantities of the docosapentaenoic acid (22:5n-6; DPA). Subjects received $4 \mathrm{~g} / \mathrm{d}$ of this microalgae oil for $4 \mathrm{wk}$ (providing $1.5 \mathrm{~g} / \mathrm{d}$ DHA and $0.6 \mathrm{~g} / \mathrm{d}$ DPA) and showed increased plasma concentrations of arachidonic acid (ARA), adrenic acid, DPA and DHA, increased DPA and DHA in erythrocyte phospholipids, increased total, LDL- and HDL-cholesterol, and increased Factor VII coagulant activity. This oil was well tolerated, without adverse effects ${ }^{(10)}$.

\section{Fish oil omega-3 fatty acids}

The consumption of equal amounts of EPA and DHA from oily fish on a weekly basis or from fish-oil capsules on a daily basis was equally effective at enriching blood lipids with $n$-3 longchain polyunsaturated fatty acids ( $n-3$ LC-PUFAs $)^{(11)}$. Fish oil $n-3$ LC-PUFAs are readily incorporated into the healthy heart and skeletal muscle membranes, and may reduce both whole-body and myocardial $\mathrm{O}_{2}$ demand during exercise, without a decrement in performance. Fish oil also increased $n-3$ LC-PUFA contents of erythrocytes ${ }^{(12)}$, lowered heart rate during incremental workloads to exhaustion, and lowered steady-state submaximal exercise heart rate and whole-body $\mathrm{O}_{2}$ consumption, but time to voluntary fatigue was not altered $^{(13)}$.

Adding DHA (fish-oils) to staple foods reduced CVD morbidity and mortality ${ }^{(14)}$, and has been recommended after myocardial infarction. Increased fish or fish-oil consumption has been associated with reduced risk of cardiac mortality, especially sudden death, by means of membrane stabilization in the cardiac myocite, inhibition of platelet aggregation, favourable modifications of the lipid profile, and decrease in systolic and diastolic blood pressure, probably due to the shift of balance between vasoconstrictive and vasodilator eicosanoids toward vasodilatation and reduction of the inflammatory response of the endothelium. Fish oil showed a propranolol-like blood pressure-lowering effect. Plasma norepinephrine and thromboxane $\mathrm{B}_{2}$ formation were likewise reduced, whereas plasma renin activity increased ${ }^{(15)}$. Dietary intervention with fish oil $n$-3 LC-PUFAs reduced plateletmonocyte aggregation, and suggested that reduced platelet activation provides a potential mechanism through which fish oils confer their cardiovascular preventative benefits ${ }^{(16)}$, and reduces atherothrombotic risk in patients with hyperlipoproteinemia ${ }^{(17)}$. 
Table 1. Description of the studies included in this review

\begin{tabular}{|c|c|c|c|c|c|c|}
\hline \multirow{2}{*}{$\begin{array}{l}\text { Dietary } \\
\text { source }\end{array}$} & \multirow[b]{2}{*}{ Participants } & \multirow[b]{2}{*}{ Design } & \multirow{2}{*}{$\begin{array}{l}n-3 \text { LC-PUFAs intervention } \\
\text { and doses }\end{array}$} & \multicolumn{2}{|c|}{ Outcomes } & \multirow[b]{2}{*}{ Reference } \\
\hline & & & & Health benefits & Health risks & \\
\hline \multicolumn{7}{|l|}{ Algae } \\
\hline & 36 overweight or obese adults & $\begin{array}{l}\text { 4.5 mo randomized con- } \\
\text { trolled double-blind trial }\end{array}$ & $2 \mathrm{~g} / \mathrm{d}$ algal DHA or placebo & $\begin{array}{l}\text { To decrease in VLDL and increase } \\
\text { in LDL and HDL particle sizes, } \\
\text { and reduction in VLDL, and total } \\
\text { TAG concentrations, blood } \\
\text { pressure and heart rate, and } \\
\text { oxidative stress }\end{array}$ & Fishy taste and eructation & Neff et al. ${ }^{(8)}$ \\
\hline & $\begin{array}{l}\text { Normolipidaemic vegetarians } \\
\text { (87 females, } 27 \text { males) }\end{array}$ & $\begin{array}{l}\text { 8-wk double-blind, pla- } \\
\text { cebo-controlled, parallel } \\
\text { design intervention } \\
\text { study }\end{array}$ & $\begin{array}{l}\text { Ulkenia sp. microalgae oil }(0.94 \mathrm{~g} \\
\text { DHA/d) }\end{array}$ & $\begin{array}{l}\text { To decrease plasma TAG, and } \\
\text { increase plasma total choles- } \\
\text { terol, LDL- and HDL-cholesterol } \\
\text { in normolipidaemic vegetarians }\end{array}$ & None & Geppert et al. ${ }^{(9)}$ \\
\hline & 39 men and 40 women & $\begin{array}{l}\text { 4-wk double-blind random- } \\
\text { ised placebo-controlled } \\
\text { parallel-design trial }\end{array}$ & $\begin{array}{l}4 \mathrm{~g} / \mathrm{d} \text { Schizochytrium sp. oil } \\
(1.5 \mathrm{~g} / \mathrm{d} \text { DHA, } 0.6 \mathrm{~g} / \mathrm{d} \text { DPA })\end{array}$ & $\begin{array}{l}\text { To increase plasma concen- } \\
\text { trations of arachidonic acid } \\
\text { (ARA), adrenic acid, DPA and } \\
\text { DHA, and DPA and DHA in } \\
\text { erythrocyte phospholipids, and } \\
\text { total, LDL- and HDL-cholesterol, } \\
\text { and increased Factor VII coagu- } \\
\text { lant activity }\end{array}$ & None & Sanders et al. ${ }^{(10)}$ \\
\hline \multirow[t]{9}{*}{ Fish oil } & & & & & & \\
\hline & $\begin{array}{l}\text { Healthy premenopausal } \\
\text { female }(21-49 \mathrm{y})\end{array}$ & $\begin{array}{l}\text { 16-wk randomized con- } \\
\text { trolled trial }\end{array}$ & $\begin{array}{l}485 \mathrm{mg} / \mathrm{d} \text { EPA and DHA from } 2 \\
\text { servings of oily fish/wk }(n 11) \text { or } \\
\text { from } 1-2 \text { capsules/d }(n 12)\end{array}$ & $\begin{array}{l}\text { EPA + DHA in RBCs increased } \\
\text { significantly more rapidly in the } \\
\text { fish group than in the capsule } \\
\text { group during the first } 4 \text { wk, but } \\
\text { rates did not differ significantly } \\
\text { between groups thereafter }\end{array}$ & None & Harris et al. ${ }^{(11)}$ \\
\hline & $\begin{array}{l}69 \text { subjects ( } 36 \text { male, } 31 \\
\text { female, mean age } 53 \mathrm{y} \text { ) with } \\
\text { fasting serum TAG } \\
\geq 1.1 \mathrm{mmol} / \mathrm{l} \text { and } 44 \\
\mathrm{BMI}>25 \mathrm{~kg} / \mathrm{m}^{2}\end{array}$ & $\begin{array}{l}\text { 12-wk randomized, } \\
\text { double-blind, placebo- } \\
\text { controlled parallel inter- } \\
\text { vention }\end{array}$ & $\begin{array}{l}\text { 2, 4, 6g/d of DHA-rich fish oil } \\
(26 \% \text { DHA, } 6 \% \text { EPA })\end{array}$ & $\begin{array}{l}\text { For every } 1 \mathrm{~g} / \mathrm{d} \text { increase in DHA } \\
\text { intake, there was a } 23 \% \\
\text { reduction in TAG, } 4.4 \% \\
\text { increase in HDL-cholesterol and } \\
7.1 \% \text { increase in LDL-chol. }\end{array}$ & None & Milte et al ${ }^{(12)}$ \\
\hline & 16 well-trained men (cyclists) & $\begin{array}{l}\text { 8-wk double-blind, parallel } \\
\text { design }\end{array}$ & $\begin{array}{l}8 \times 1 \mathrm{~g} \text { capsules } / \mathrm{d} \text { olive oil (con- } \\
\text { trol) or fish oil }\end{array}$ & $\begin{array}{l}\text { To lower heart rate (including } \\
\text { peak heart rate) during incre- } \\
\text { mental workloads to exhaustion, } \\
\text { steady-state submaximal exer- } \\
\text { cise heart rate, whole-body } \mathrm{O}_{2} \\
\text { consumption, and heart press- } \\
\text { ure product }\end{array}$ & None & Peoples et al. ${ }^{(13)}$ \\
\hline & $\begin{array}{l}213 \text { middle-aged men and } \\
\text { women with untreated elev- } \\
\text { ated total cholesterol or } \\
\text { blood pressure }\end{array}$ & $\begin{array}{l}\text { 5-wk double-blind, factorial } \\
\text { placebo-controlled ran- } \\
\text { domized trial }\end{array}$ & $\begin{array}{l}\text { bread, cereal bars and cracker } \\
\text { biscuits fortified with } 2 \mathrm{~g} \text { fish oils } \\
\text { (DHA) }\end{array}$ & $\begin{array}{l}\text { To reduce CVD morbidity and } \\
\text { mortality }\end{array}$ & None & Harrison et al. ${ }^{(14)}$ \\
\hline & $\begin{array}{l}47 \text { male patients with mild } \\
\text { hypertension }\end{array}$ & $\begin{array}{l}\text { 40-wk randomized, } \\
\text { double-blind, placebo- } \\
\text { controlled intervention }\end{array}$ & $\begin{array}{l}\text { fish oil capsules }(9 \mathrm{~g} / \mathrm{d} \text { equivalent } \\
\text { to } 1.8 \mathrm{~g} / \mathrm{d} \text { of EPA and } 1.1 \mathrm{~g} / \mathrm{d} \text { of } \\
\text { DHA) }\end{array}$ & $\begin{array}{l}\text { To decrease plasma norepi- } \\
\text { nephrine and thromboxane } B_{2} \\
\text { formation, and increase plasma } \\
\text { renin activity }\end{array}$ & None & Singer et al. ${ }^{(15)}$ \\
\hline & 14 male adults & $\begin{array}{l}\text { 4-wk double-blind interven- } \\
\text { tion, parallel design }\end{array}$ & $500 \mathrm{~g} / \mathrm{d}$ oil rich fish & $\begin{array}{l}\text { Inverse correlation between plate- } \\
\text { let-monocyte aggregation and } \\
\text { plasma omega-3 fatty acid con- } \\
\text { centrations }\end{array}$ & None & Din et al. ${ }^{(16)}$ \\
\hline & $\begin{array}{l}26 \text { male hypercholesterolemic } \\
\text { patients }\end{array}$ & $\begin{array}{l}\text { 6-wk placebo-controlled, } \\
\text { double-blind intervention }\end{array}$ & $\begin{array}{l}216 \mathrm{mg} / \mathrm{d} \mathrm{EPA}, 140 \mathrm{mg} / \mathrm{d} \text { DHA, } \\
390 \mathrm{mg} / \mathrm{d} \text { gamma-LA, } \\
3480 \mathrm{mg} / \mathrm{d} \mathrm{LA}\end{array}$ & $\begin{array}{l}\text { To decrease MDA formation, total, } \\
\text { and LDL cholesterol, triglycer- } \\
\text { ides, and platelet activation }\end{array}$ & None & Pirich et al..$^{(17)}$ \\
\hline & $\begin{array}{l}84 \text { patients with low self- } \\
\text { reported fish intake } \\
\text { accepted for cardiac surgery } \\
\text { (cardiopulmonary bypass) }\end{array}$ & $\begin{array}{l}\text { 63-d randomized, double- } \\
\text { blind intervention, paral- } \\
\text { lel design }\end{array}$ & $\begin{array}{l}\text { fish oil }(6 \mathrm{~g} \mathrm{EPA}+\mathrm{DHA} / \mathrm{d}) \text { for } \\
\text { either } 7,14 \text {, or } 21 \mathrm{~d} \text { before sur- } \\
\text { gery }\end{array}$ & $\begin{array}{l}n-3 \text { fatty acids are rapidly incorpor- } \\
\text { ated into human myocardial } \\
\text { phospholipids at the expense of } \\
\text { ARA }\end{array}$ & None & Metcalf et al. ${ }^{(18)}$ \\
\hline
\end{tabular}




\begin{tabular}{ll}
\hline $\begin{array}{l}\text { Dietary } \\
\text { source }\end{array}$ & Participants \\
\hline 14 patients heart failure \\
\\
107 patients percutaneous \\
transluminal coronary angio- \\
plasty \\
\\
\\
229 postmenopausal women \\
with coronary artery disease \\
\\
\\
60 patients with coronary \\
heart disease \\
4680 men and women (40- \\
$59 \mathrm{y})$ from 17 population- \\
based samples in China, \\
Japan, United Kingdom, \\
and United States \\
44720 participants
\end{tabular}

1148 subjects

20 health subjects (10 women)

102 patients with confirmed stroke

11 subjects with epilepsy

Design

18-wk randomized, placebo-controlled, double-

vention

6-mo randomized, plablind intervention

\section{$1-y$ prospective cohort
study}

6-wk double-blinded intervention study

Cross-sectional epidemiologic study

6-y follow up of Women's Health Initiative clinica trials

Meta-analysis of random ized double-blind, placebo-controlled, paralel-group trials

Randomized, placebo-controlled, double-blind, crossover study

\section{2-wk randomized con-} trolled trial

Randomized, double-blind two-period crossover clinical trial.
Outcomes

$n$-3 LC-PUFAs intervention

Health benefits

Health risks

Reference

To decrease TNF- $\alpha$ production in heart failure and improve body weight

10 capsules of fish oil $(3 \mathrm{~g} / \mathrm{d}$, containing $1.8 \mathrm{~g}$ EPA, $1.2 \mathrm{~g} \mathrm{DHA}$, $n 58$ ) besides aspirin and calcium blockers, beginning $4.3 \mathrm{~d}$ (control group just aspirin and calcium blockers, $n$ 49) before

coronary angioplasty
Fish and $n-3$ PUFA intake by food frequency questionnaire

Consumption of $\geq 2$ servings of fish or $\geq 1$ serving of tuna or dark fish per week was associated with smaller increases in the percentage of stenosis

Significant reductions of serum triglycerides and of SVCAM- 1 and IL-6

$00 \mathrm{~g} / \mathrm{wk}$ of different Atlantic salmon

Association between $n$-3 LCPUFAs intake and blood pressure (four 24-h recalls)

nverse relation of $n-3$ LC-PUFA intake and blood pressure

Association between fish oil intake and atrial fibrillation (food frequency questionnaire)

$2 \mathrm{~g} / \mathrm{d}$ (4 capsules) fish oil (961 mg $n$-3 LC-PUFAs: $464 \mathrm{mg}$ EPA, $335 \mathrm{mg}$ DHA, and $162 \mathrm{mg}$ other $n$-3 LC-PUFAs), $12 \mathrm{mo} ; 1.8 \mathrm{~g} / \mathrm{d}$ fish oil (42\% EPA, $30 \%$ DHA) cid $12 \%$ palmitic $73 \%$ EPA/DHA), $2 y$

Participants reported to the tory on 2 separate days and received 1 of 2 treatment conditions: High-fat meal (1 g EPA and DHA from fish oil supplement) or high-fat meal with placebo (lactose capsules). Each visit was separated by at east $72 \mathrm{~h}$ but no more than 14 days. $3 \mathrm{~g} / \mathrm{d}$ encaps

total omega-3: 0.7 $\mathrm{DHA} ; 0.3$ EPA) capsules/d; $9600 \mathrm{mg}$ of fish $(2880 \mathrm{mg} / \mathrm{d}$ of $n-3$ LC-PUFAs: $1728 \mathrm{mg} / \mathrm{d} \mathrm{EPA}+1152 \mathrm{mg} / \mathrm{d}$ DHA) or soybean oil (placebo): 4-wk washout period; 12-wk treatment crossover.
No evidence of an association between fish or omega-3 fatty fibrillation

Findings do not support a protective effect of $n-3$ LC-PUFAs

Brachial artery flow-mediated dilation remained unchanged, and resting forearm blood flow and

Fahs et al. ${ }^{(26)}$

No effects on CVD biomarkers or mood in patients with ischemic stroke

To decrease seizure severity and triglycerides, and increase HDL. acid intake and incident atrial
Mehra et al. ${ }^{(19)}$

Kaul et al. (20)

None

None

Erkkilä et al. ${ }^{(21)}$

Seierstad et al.(22)

Ueshima et al. ${ }^{(23)}$

Berry et al. ${ }^{(24)}$

Brouwer et al. ${ }^{(25)}$ 
Table 1. Continued

\begin{tabular}{|c|c|c|c|c|c|c|}
\hline \multirow{2}{*}{$\begin{array}{l}\text { Dietary } \\
\text { source }\end{array}$} & \multirow[b]{2}{*}{ Participants } & \multirow[b]{2}{*}{ Design } & \multirow{2}{*}{$\begin{array}{l}n-3 \text { LC-PUFAs intervention } \\
\text { and doses }\end{array}$} & \multicolumn{2}{|c|}{ Outcomes } & \multirow[b]{2}{*}{ Reference } \\
\hline & & & & Health benefits & Health risks & \\
\hline & $\begin{array}{l}17 \text { healthy middle-aged } \\
(35-64 \text { y) subjects }\end{array}$ & $\begin{array}{l}\text { Randomized, placebo-con- } \\
\text { trolled, double-blind, } \\
\text { crossover study }\end{array}$ & $\begin{array}{l}\text { 4-wk intervention (fish oil capsules } \\
\text { with every meal, } 1260 \mathrm{mg} \text { EPA, } \\
540 \mathrm{mg} \text { DHA) or placebo and } \\
\text { 4-wk wash out }\end{array}$ & $\begin{array}{l}\text { No changes in the fatty acid com- } \\
\text { position of plasma and erythro- } \\
\text { cyte phospholipids }\end{array}$ & None & Watanabe et al. ${ }^{(29)}$ \\
\hline & $\begin{array}{l}16 \text { normolipidemic subjects } \\
\text { (9 women) }\end{array}$ & $\begin{array}{l}\text { 8-wk randomized con- } \\
\text { trolled trial }\end{array}$ & $\begin{array}{l}6 \mathrm{~g} / \mathrm{d} \text { (6 capsules) fish oil (3g } n-3 \\
\text { LC-PUFAs) }\end{array}$ & $\begin{array}{l}\text { Increase of total glutathione, } \\
\text { homocystein, and NO plasma } \\
\text { concentrations. }\end{array}$ & None & Piolot et al. ${ }^{(30)}$ \\
\hline & $\begin{array}{l}12 \text { T2DM normotriglyceridemic } \\
\text { subjects without insulin } \\
\text { treatment }\end{array}$ & $\begin{array}{l}\text { 9-wk two-armed, parallel, } \\
\text { placebo-controlled, ran- } \\
\text { domized }\end{array}$ & $\begin{array}{l}5.9 \mathrm{~g} / \mathrm{d} n-3 \text { LC-PUFAs }(1 \cdot 8 \mathrm{~g} \\
20: 5 n-3,3 \cdot 0 \mathrm{~g} 22: 6 n-3)\end{array}$ & $\begin{array}{l}\text { Decrease of VLDL and increase of } \\
\text { HDL size particles, increase of } \\
\text { small LDL concentration, and } \\
\text { no effect on oxidized LDL }\end{array}$ & None & Mostad et al. ${ }^{(31)}$ \\
\hline & $\begin{array}{l}200 \text { mildly hypercholesterolae- } \\
\text { mic Indian adults aged } \\
35-55 \mathrm{y}\end{array}$ & $\begin{array}{l}\text { 4-wk } 2 \times 2 \text { factorial, } \\
\text { double-blind controlled } \\
\text { trial }\end{array}$ & $\begin{array}{l}\text { Once-a-day yoghurt drink ( } 2 \mathrm{~g} / \mathrm{d} \\
\text { plant sterols) and capsules } \\
(2 \mathrm{~g} / \mathrm{d} \text { fish oil } n-3 \text { LC-PUFAs })\end{array}$ & $\begin{array}{l}\text { Reduction in LDL-chol. and trigly- } \\
\text { cerides, and increase in HDL- } \\
\text { chol. concentrations }\end{array}$ & None & Khandelwal et al. ${ }^{(32)}$ \\
\hline & 338 adult men & $\begin{array}{l}\text { 30-y follow-up survey of } \\
\text { the Dutch and Finnish } \\
\text { cohorts of the Seven } \\
\text { Countries Study }\end{array}$ & $\begin{array}{l}\text { Intake of total, saturated, and } \\
\text { monounsaturated fatty acids } \\
\text { and dietary cholesterol } 20 \mathrm{y} \\
\text { before diagnosis }\end{array}$ & $\begin{array}{l}\text { High intake of fat, especially that } \\
\text { of saturated fatty acids, contrib- } \\
\text { utes to the risk of glucose intol- } \\
\text { erance and T2DM }\end{array}$ & None & Feskens et al. ${ }^{(33)}$ \\
\hline & $\begin{array}{l}25639 \text { men and women } \\
(40-79 y)\end{array}$ & $\begin{array}{l}\text { 10-y follow-up survey of } \\
\text { the EPIC-Norfolk Study }\end{array}$ & $\begin{array}{l}\text { Assessment of fish and seafood } \\
\text { intake by means of FFQ }\end{array}$ & $\begin{array}{l}\text { Higher total fish intake ( } \geq 1 \text { vs }<1 \\
\text { portions/wk) was associated } \\
\text { with a lower risk of diabetes }\end{array}$ & None & Patel et al. ${ }^{(34)}$ \\
\hline & $\begin{array}{l}175 \text { men and women } \\
(64-87 \mathrm{y})\end{array}$ & 4-y follow-up survey & $\begin{array}{l}\text { Assessment of fish and seafood } \\
\text { intake by means of cross-check } \\
\text { dietary history method }\end{array}$ & $\begin{array}{l}\text { In elderly population, the habitual } \\
\text { consumption of a small amount } \\
\text { of fish may protect against the } \\
\text { development of impaired glu- } \\
\text { cose tolerance and T2DM }\end{array}$ & None & Feskens et al. ${ }^{(35)}$ \\
\hline & $\begin{array}{l}3 \text { cohort studies. NHS (1976; } \\
121700 \text { female nurses } \\
30-55 \text { y at baseline); NHS2 } \\
\text { (1989; } 116609 \text { female } \\
\text { nurses } 26-46 \text { y at baseline); } \\
\text { and HPFS (1986; } 51529 \\
\text { male health care pro- } \\
\text { fessionals } 39-78 \text { y at base- } \\
\text { line) }\end{array}$ & $\begin{array}{l}\text { Large cohort follow-up sur- } \\
\text { veys }\end{array}$ & $\begin{array}{l}\text { Assessed of diet using FFQ, admi- } \\
\text { nistered at 4-y intervals during } \\
\text { the follow-up period }\end{array}$ & $\begin{array}{l}\text { No evidence that higher consump- } \\
\text { tion of LC-PUFA and fish } \\
\text { reduces the risk of T2DM }\end{array}$ & None & Kaushik et al. ${ }^{(36)}$ \\
\hline & 8 T2DM male subjects & $\begin{array}{l}8 \text { wk randomized con- } \\
\text { trolled trial }\end{array}$ & $\begin{array}{l}8 \mathrm{~g} / \mathrm{d} n-3 \text { LC-PUFAs, as marine- } \\
\text { lipid concentrate capsules }\end{array}$ & $\begin{array}{l}\text { Triglyceride and cholesterol } \\
\text { plasma levels decreased, no } \\
\text { alteration of HDL-chol. levels, } \\
\text { but increased fasting and meal.- } \\
\text { stimulated glucose concen- } \\
\text { trations. }\end{array}$ & $\begin{array}{l}\text { Marine-lipid concentrate } \\
\text { capsules supplying } \\
\text { large amounts of } n-3 \\
\text { LC-PUFAs should be } \\
\text { used cautiously in the } \\
\text { T2DM patient }\end{array}$ & Friday et al. ${ }^{(37)}$ \\
\hline & 162 healthy individuals & $\begin{array}{l}3 \text { mo randomized placebo- } \\
\text { controlled trial }\end{array}$ & $3.6 \mathrm{~g} / \mathrm{d} n-3$ LC-PUFAs & $\begin{array}{l}\text { Moderate supplementation of fish } \\
\text { oil does not affect insulin sensi- } \\
\text { tivity, insulin secretion, beta-cell } \\
\text { function or qlucose tolerance }\end{array}$ & None & Rivellese et al. ${ }^{(38)}$ \\
\hline & 12 T2DM men & $\begin{array}{l}\text { Randomized, double-blind, } \\
\text { crossover study }\end{array}$ & $\begin{array}{l}6 \mathrm{~g} / \mathrm{d} \text { of either fish oil or sunflower } \\
\text { oil, separated by a 2-mo wash- } \\
\text { out interval }\end{array}$ & $\begin{array}{l}\text { Moderate dose of fish oil did not } \\
\text { lead to deleterious effects on } \\
\text { glycemic control or whole-body } \\
\text { insulin sensitivity in T2DM men, } \\
\text { with preserved TAG lowering } \\
\text { capacities }\end{array}$ & None & Luo et al. ${ }^{(39)}$ \\
\hline & 10 T2DM subjects (42-65y) & $\begin{array}{l}\text { Randomized, double-blind, } \\
\text { crossover study }\end{array}$ & $\begin{array}{l}\text { No supplementation (baseline); } \\
10 \mathrm{~g} / \mathrm{d} \text { fish oil concentrate }(30 \% \\
\text { omega } 3 \mathrm{FAs}) ; 10 \mathrm{~g} / \mathrm{d} \text { safflower } \\
\text { oil; over separate } 3 \text {-wk periods }\end{array}$ & $\begin{array}{l}\text { Dietary fish oil supplementation } \\
\text { adversely affected glycemic } \\
\text { control in T2DM subjects with- } \\
\text { out producing significant ben- } \\
\text { eficial effects on plasma lipids }\end{array}$ & None & Borkman et al. ${ }^{(40)}$ \\
\hline
\end{tabular}




\begin{tabular}{|c|c|c|c|c|c|c|}
\hline \multirow{2}{*}{$\begin{array}{l}\text { Dietary } \\
\text { source }\end{array}$} & \multirow[b]{2}{*}{ Participants } & \multirow[b]{2}{*}{ Design } & \multirow{2}{*}{$\begin{array}{l}n-3 \text { LC-PUFAs intervention } \\
\text { and doses }\end{array}$} & \multicolumn{2}{|c|}{ Outcomes } & \multirow[b]{2}{*}{ Reference } \\
\hline & & & & Health benefits & Health risks & \\
\hline & $\begin{array}{l}36328 \text { women (mean age } \\
54.6 \mathrm{y})\end{array}$ & $\begin{array}{l}\text { 12.4-y follow-up survey of } \\
\text { the Women's Health } \\
\text { Study (1992-2008) }\end{array}$ & $\begin{array}{l}\text { Assessment of fish and seafood } \\
\text { intake by means of FFQ and } \\
\text { self-reported of T2DM }\end{array}$ & n.d. & $\begin{array}{l}\text { Increased risk of T2DM } \\
\text { with the intake of } n-3 \\
\text { LC-PUFAs, especially } \\
\text { with higher intakes } \\
(\geq 0 \cdot 20 \mathrm{~g} / \mathrm{d} \text { of } n-3 \text { or } \geq 2 \\
\text { servings } / \mathrm{d} \text { of fish) }\end{array}$ & Djoussé et al. ${ }^{(41)}$ \\
\hline & 2397 participants & $\begin{array}{l}\text { Cross-sectional and } 1-y \\
\text { longitudinal, multicenter } \\
\text { randomized trial (Look } \\
\text { AHEAD survey) }\end{array}$ & $\begin{array}{l}\text { Assessment of fish and seafood } \\
\text { intake by means of FFQ }\end{array}$ & $\begin{array}{l}\text { Marine } n-3 \text { LC-PUFAs intake is } \\
\text { inversely associated triglycer- } \\
\text { ides and weakly with HDL-chol. }\end{array}$ & None & Belalcazar et al..$^{(42)}$ \\
\hline & 162 healthy individuals & $\begin{array}{l}\text { 3-mo randomized placebo- } \\
\text { controlled trial }\end{array}$ & $3.6 \mathrm{~g} / \mathrm{d}$ fish oil $n-3$ LC-PUFAs & $\begin{array}{l}\text { Moderate supplementation of fish } \\
\text { oil does not affect insulin sensi- } \\
\text { tivity, insulin secretion, beta-cell } \\
\text { function or glucose tolerance }\end{array}$ & None & Giacco et al. ${ }^{(43)}$ \\
\hline & $\begin{array}{l}12 \text { normotriglyceride T2DM } \\
\text { subjects }\end{array}$ & $\begin{array}{l}\text { 9-wk two-armed, parallel, } \\
\text { placebo-controlled, ran- } \\
\text { domized trial }\end{array}$ & $\begin{array}{l}5.9 \mathrm{~g} / \mathrm{d} \text { fish oil }(1 \cdot 8 \mathrm{~g} 20: 5 n-3 \\
3.0 \mathrm{~g} 22: 6 n-3)\end{array}$ & $\begin{array}{l}\text { To increase HDL size and small } \\
\text { LDL concentration, no effect on } \\
\text { oxidized LDL, and decreased } \\
\text { insulin sensitivity }\end{array}$ & None & Mostad et al. ${ }^{(44)}$ \\
\hline & $\begin{array}{l}25 \text { young iron-deficient women } \\
(18-30 \mathrm{y})\end{array}$ & $\begin{array}{l}\text { 9-wk randomised cross- } \\
\text { over dietary intervention } \\
\text { study }\end{array}$ & $\begin{array}{l}\text { Diet contained } 2 \text { portions of sal- } \\
\text { mon, } 2 \text { cans of water-packed } \\
\text { tuna ( } 56 \mathrm{~g} \text { each), } 1 \text { can of sar- } \\
\text { dines in olive oil, } 1 \text { portion of } \\
\text { lean fish, } 1 \text { portion of red meat, } \\
2 \text { portions of poultry and } 2 \text { eggs } \\
\text { per week }\end{array}$ & $\begin{array}{l}\text { Insulin levels significantly } \\
\text { decreased and insulin and HDL- } \\
\text { chol. sensitivity significantly } \\
\text { increased }\end{array}$ & None & $\begin{array}{l}\text { Navas-Carretero } \\
\text { et al. }{ }^{(45)}\end{array}$ \\
\hline & 42 healthy subjects & $\begin{array}{l}4 \text { wk randomized placebo- } \\
\text { controlled trial }\end{array}$ & $\begin{array}{l}\text { Fish oil rich EPA }(4.7 \mathrm{~g} / \mathrm{d}) \text {, or DHA } \\
(4.9 \mathrm{~g} / \mathrm{d})\end{array}$ & $\begin{array}{l}\text { Supplementation with DHA, but } \\
\text { not with EPA, suppresses T } \\
\text { lymphocyte activation. EPA } \\
\text { alone does not, therefore, influ- } \\
\text { ence CD69 expression }\end{array}$ & None & Kew et al. ${ }^{(46)}$ \\
\hline & $\begin{array}{l}324 \text { subjects }(20-40 \mathrm{y}) \text { with } \\
\text { BMI } 27.5-32.5 \mathrm{~kg} / \mathrm{m}^{2}\end{array}$ & $\begin{array}{l}\text { 8-wk randomized } \\
\text { controlled trial }\end{array}$ & $\begin{array}{l}\text { salmon }(3 \times 150 \mathrm{~g} / \mathrm{wk}, 2 \cdot 1 \mathrm{~g} / \mathrm{d} n-3 \\
\text { LC-PUFAs); cod }(3 \times 150 \mathrm{~g} / \mathrm{wk} \text {, } \\
0.3 \mathrm{~g} / \mathrm{d} n-3 \mathrm{LC}-\mathrm{PUFA}) ; \text { fish oil } \\
\text { capsules (1.3 g/d } n-3 \text { LC- } \\
\text { PUFAs); control (sunflower oil } \\
\text { capsules, no seafood) }\end{array}$ & $\begin{array}{l}\text { Subjects that ate fish experienced } \\
\text { weight loss, and decreases tri- } \\
\text { glyceride levels, in inflammation } \\
\text { parameters, and systolic and } \\
\text { diastolic blood pressure. Sal- } \\
\text { mon consumption was the most } \\
\text { effective. Body weight, leptin } \\
\text { and insulin levels decreased, } \\
\text { and ghrelin increased. Weight } \\
\text { loss explained the effects of } \\
\text { fatty seafood on leptin and } \\
\text { ghrelin, but not insulin }\end{array}$ & None & $\begin{array}{l}\text { Ramel et al. }{ }^{(47,48,75)} \\
\text { Gunnarsdottir } \\
\text { et al. } .^{(79)}\end{array}$ \\
\hline & 92 male subjects ( $35-70 y)$ & $\begin{array}{l}\text { 8-wk randomized, parallel- } \\
\text { arm, food-based inter- } \\
\text { vention study }\end{array}$ & $\begin{array}{l}\text { Lunches with pork/chicken/beef ( } n \\
\text { 30); freshwater fish ( } n \text { 30); or } \\
\text { oily fish ( } n \text { 32) }\end{array}$ & $\begin{array}{l}\text { Reduced serum levels of triglycer- } \\
\text { ides and interleukin-6 and } \\
\text { increased levels of HDL-chol. }\end{array}$ & None & Zhang et al. ${ }^{(49)}$ \\
\hline & $\begin{array}{l}10 \text { elite athletes with and } 10 \\
\text { without exercise-induced } \\
\text { bronchoconstriction }\end{array}$ & $\begin{array}{l}\text { 3-wk randomized double- } \\
\text { blind crossover study }\end{array}$ & $\begin{array}{l}\text { Fish oil capsules ( } 3 \cdot 2 \mathrm{~g} / \mathrm{d} \text { EPA; } \\
2 \cdot 2 \mathrm{~g} / \mathrm{d} \text { DHA) or placebo cap- } \\
\text { sules (olive oil) }\end{array}$ & $\begin{array}{l}\text { No effect on preexercise pulmon- } \\
\text { ary function, but improved post- } \\
\text { exercise pulmonary function, } \\
\text { and decreased leukotriene, } 9 \alpha, \\
\text { and } 11 \beta \text {-prostaglandin, TNF } \alpha, \\
\text { interleukin-1 } \beta\end{array}$ & None & Mickleborough et al. ${ }^{(50)}$ \\
\hline & $\begin{array}{l}36 \text { patients with end-stage } \\
\text { renal disease }\end{array}$ & $\begin{array}{l}\text { 6-mo double-blind, per- } \\
\text { muted-randomized, and } \\
\text { placebo-controlled trial }\end{array}$ & $\begin{array}{l}2 \text { soft-gel pills } / \mathrm{d}(1 \mathrm{~g} \text { each) of fish } \\
\text { oil supplements }(960 \mathrm{mg} / \mathrm{d} \text { EPA; } \\
600 \mathrm{mg} / \mathrm{d} \mathrm{DHA})\end{array}$ & Decrease of C-RP levels & None & Bowden et al..$^{(51)}$ \\
\hline
\end{tabular}


Table 1. Continued

\begin{tabular}{|c|c|c|c|c|c|c|}
\hline \multirow{2}{*}{$\begin{array}{l}\text { Dietary } \\
\text { source }\end{array}$} & \multirow[b]{2}{*}{ Participants } & \multirow[b]{2}{*}{ Design } & \multirow{2}{*}{$\begin{array}{l}n-3 \text { LC-PUFAs intervention } \\
\text { and doses }\end{array}$} & \multicolumn{2}{|c|}{ Outcomes } & \multirow[b]{2}{*}{ Reference } \\
\hline & & & & Health benefits & Health risks & \\
\hline & $\begin{array}{l}33 \text { subjects with myocardial } \\
\text { infarction or unstable } \\
\text { ischemic attack }\end{array}$ & $\begin{array}{l}\text { 8-wk randomized, parallel- } \\
\text { arm, food-based clinical } \\
\text { trial }\end{array}$ & $\begin{array}{l}\text { Lunches with fatty fish ( } n \text { 11), lean } \\
\text { fish }(n \text { 12) or control (lean beef, } \\
\text { pork and chicken) }(n \text { 10), } 4 \\
\text { meals/wk }\end{array}$ & $\begin{array}{l}\text { Decrease of lipids which are } \\
\text { potential mediators of lipid- } \\
\text { induced insulin resistance and } \\
\text { inflammation }\end{array}$ & None & Lankinen et al. ${ }^{(52)}$ \\
\hline & 727 women $(43-69 y)$ & $\begin{array}{l}\text { Cross-sectional survey of } \\
\text { a cohort of the Nurses' } \\
\text { Health Study I }\end{array}$ & $\begin{array}{l}\text { Assessment of fish and seafood } \\
\text { intake (and } n-3 \text { LC-PUFAs) by } \\
\text { means of FFQ }\end{array}$ & $\begin{array}{l}n \text { - } 3 \text { LC-PUFAs intake were inver- } \\
\text { sely related to sICAM- } 1 \text { and } \\
\text { sVCAM- } 1 \text {, CRP, and E-selectin. }\end{array}$ & None & López-García et al. ${ }^{(53)}$ \\
\hline & $\begin{array}{l}60 \text { patients with severe acute } \\
\text { pancreatitis }\end{array}$ & $\begin{array}{l}\text { 2-wk randomized con- } \\
\text { trolled trial }\end{array}$ & $\begin{array}{l}\text { Intravenous supplementation with } \\
\text { fish oil } n-3 \text { LC-PUFAs emulsion } \\
(0.2 \mathrm{~g} / \mathrm{kg} / \mathrm{d})\end{array}$ & $\begin{array}{l}\text { Parenteral supplementation with } \\
\text { omega-3 fish oil emulsion lower } \\
\text { the magnitude and persistence } \\
\text { time of the systemic inflamma- } \\
\text { tory response syndrome }\end{array}$ & None & Xiong et al. ${ }^{(54)}$ \\
\hline & $\begin{array}{l}64 \text { patients with stable mild } \\
\text { rheumatoid arthritis }\end{array}$ & $\begin{array}{l}\text { 12-mo randomized double- } \\
\text { blind placebo controlled } \\
\text { trial }\end{array}$ & $\begin{array}{l}10 \text { capsules } / \mathrm{d} \text { ( } 171 \mathrm{mg} / \text { capsule } \\
\text { EPA, and } 114 / \mathrm{mg} \text { capsule DHA) }\end{array}$ & $\begin{array}{l}\text { Significant reduction in non-ster- } \\
\text { oidal anti-inflammatory drug } \\
\text { requirement from month 3rd }\end{array}$ & None & Lau et al. ${ }^{(55)}$ \\
\hline & $\begin{array}{l}250 \text { patients with nonsurgical } \\
\text { neck or back pain }\end{array}$ & $\begin{array}{l}\text { 4-mo randomized con- } \\
\text { trolled trial }\end{array}$ & Fish oil EPA + DHA $(1200 \mathrm{mg} / \mathrm{d})$ & $\begin{array}{l}n-3 \text { LC-PUFAs supplementation } \\
\text { showed equivalent effect of ibu- } \\
\text { profen in reducing arthritic pain }\end{array}$ & None & Maroon et al. ${ }^{(56)}$ \\
\hline & $\begin{array}{l}97 \text { patients with rheumatoid } \\
\text { arthritis }\end{array}$ & $\begin{array}{l}\text { 9-mo randomized, pro- } \\
\text { spective, investigator- } \\
\text { initiated dual centre, } \\
\text { double-blind placebo- } \\
\text { controlled trial }\end{array}$ & $\begin{array}{c}10 \mathrm{~g} / \mathrm{d} \text { (10 capsules) of cod liver oil } \\
(150 \mathrm{mg} \text { EPA,70 } \mathrm{mg} \text { DHA) }\end{array}$ & $\begin{array}{l}\text { Cod liver oil supplements contain- } \\
\text { ing } n-3 \text { LC-PUFAs decreased } \\
\text { non-steroidal anti-inflammatory } \\
\text { drug requirement }\end{array}$ & None & Galarraga et al..(57) \\
\hline & $\begin{array}{l}19 \text { patients with rheumatoid } \\
\text { arthritis }\end{array}$ & $\begin{array}{l}2 \text { y randomized controlled } \\
\text { trial }\end{array}$ & $\begin{array}{l}\text { Intervention: 1) Fish oil EPA } \\
2.7 \mathrm{~g} / \mathrm{d} \text { and DHA } 1.8 \mathrm{~g} / \mathrm{d} \\
\text { 2) Fish oil EPA } 270 \mathrm{mg} / \mathrm{d} \text { and } \\
\text { DHA } 180 \mathrm{mg} / \mathrm{d}\end{array}$ & $\begin{array}{l}\text { EPA increases ciclooxygenase } \\
\text { inhibitory activity of paracetamol } \\
\text { in rheumatoid arthritis patients }\end{array}$ & None & Caughey et al. ${ }^{(58)}$ \\
\hline & 12 asthmatic patients & $\begin{array}{l}\text { 1-y randomized double- } \\
\text { blind placebo controlled } \\
\text { trial }\end{array}$ & $1 \mathrm{~g} / \mathrm{d}$ EPA + DHA (Liparmony $\mathrm{I}^{\circledR}$ ) & $\begin{array}{l}\text { A positive effect on forced expira- } \\
\text { tory volume in } 1 \mathrm{~s} \text { was observed } \\
\text { after the } 9 \text { month of treatment }\end{array}$ & None & Dry et al. ${ }^{(59)}$ \\
\hline & $\begin{array}{l}216 \text { adult participants } \\
(54 \pm 12 \mathrm{y} ; 47 \% \text { female })\end{array}$ & $\begin{array}{l}\text { 6-mo multi-center, parallel, } \\
\text { randomized, controlled } \\
\text { intervention study }\end{array}$ & $\begin{array}{l}\text { Two 150-g portions/wk (salmon or } \\
\text { cod) }\end{array}$ & $\begin{array}{l}\text { Serum CRP concentrations were } \\
\text { lower after salmon and cod con- } \\
\text { sumption, but exploratory anal- } \\
\text { ysis of local markers of } \\
\text { inflammation in the colon or } \\
\text { faeces did not reveal this effect }\end{array}$ & None & Pot et al. ${ }^{(60)}$ \\
\hline & $\begin{array}{l}15 \text { healthy men }(26 \pm 3 y) \text { BMI } \\
23.8 \pm 1.9 \mathrm{~kg} / \mathrm{m}^{2}\end{array}$ & $\begin{array}{l}3-4 \text { wk randomized pla- } \\
\text { cebo controlled trial }\end{array}$ & $\begin{array}{l}7.2 \mathrm{~g} / \mathrm{d} \text { fish oil, (providing } 1.1 \mathrm{~g} / \mathrm{d} \\
20: 5(n-3) \text { and } 0.7 \mathrm{~g} / \mathrm{d} 22: 6 \\
(n-3) \text { fatty acids) }\end{array}$ & $\begin{array}{l}\text { Fish oil exert beneficial effects in } \\
\text { sepsis though non-inflammatory }\end{array}$ & None & Michaeli et al. ${ }^{(61)}$ \\
\hline & $\begin{array}{l}302 \text { participants }(167 \text { men; } \\
135 \text { women; } 66-80 \mathrm{y})\end{array}$ & $\begin{array}{l}26 \text { wk randomized double- } \\
\text { blind controlled trial }\end{array}$ & $\begin{array}{l}\text { Intervention: 1) } 1.8 \mathrm{~g} \mathrm{EPA}+ \\
\mathrm{DHA} / \mathrm{d} \text { 2) } 0.4 \mathrm{~g} \mathrm{EPA}+\mathrm{DHA} / \mathrm{d} 3 \text { ) } \\
4.0 \mathrm{~g} \text { high-oleic acid sunflower } \\
\text { oil. }\end{array}$ & $\begin{array}{l}\text { EPA + DHA intake changed the } \\
\text { expression of } 1040 \text { genes, and } \\
\text { decreased expression of genes } \\
\text { involved in inflammatory- and } \\
\text { atherogenic-related pathways }\end{array}$ & None & Bouwens et al. ${ }^{(62)}$ \\
\hline & 36 girls $(18-22 y)$ & $\begin{array}{l}\text { 3-mo randomized placebo } \\
\text { controlled trial }\end{array}$ & $\begin{array}{l}15 \mathrm{~mL} / \mathrm{d} \text { fish oil ( } 550 \mathrm{mg} \text { EPA; } \\
205 \mathrm{mg} \text { DHA) }\end{array}$ & $\begin{array}{l}\text { Marked reduction in low back pain } \\
\text { and abdominal pain, and fewer } \\
\text { rescue doses of ibuprofen }\end{array}$ & None & Moghadamnia et al. ${ }^{(63)}$ \\
\hline & $\begin{array}{l}270 \text { Pregnant women } \\
(18-41 \mathrm{y})\end{array}$ & $\begin{array}{l}\text { Randomized placebo con- } \\
\text { trolled trial }\end{array}$ & $\begin{array}{l}\text { Intervention from } 22 \text { wk to delivery: } \\
\text { 1) modified fish oil; } \\
\text { 2) } 5 \text {-methyl-tetrahydro-folate } \\
\text { 3) both; 4) placebo }\end{array}$ & $\begin{array}{l}\text { Fish oil supplementation during } \\
\text { the second half of pregnancy } \\
\text { appears not to decrease antioxi- } \\
\text { dant status }\end{array}$ & None & Franke et al. ${ }^{(64)}$ \\
\hline
\end{tabular}




\begin{tabular}{|c|c|c|c|c|c|c|}
\hline \multirow{2}{*}{$\begin{array}{l}\text { Dietary } \\
\text { source }\end{array}$} & \multirow[b]{2}{*}{ Participants } & \multirow[b]{2}{*}{ Design } & \multirow{2}{*}{$\begin{array}{l}n-3 \text { LC-PUFAs intervention } \\
\text { and doses }\end{array}$} & \multicolumn{2}{|c|}{ Outcomes } & \multirow[b]{2}{*}{ Reference } \\
\hline & & & & Health benefits & Health risks & \\
\hline & $\begin{array}{l}98 \text { pregnant women and their } \\
\text { infants assessed at } 2.5 \mathrm{y}\end{array}$ & $\begin{array}{l}\text { Randomized double-blind } \\
\text { placebo controlled trial }\end{array}$ & $\begin{array}{l}\text { Intervention from } 20 \text { wk to delivery: } \\
\text { Fish oil ( } 2 \cdot 2 \mathrm{~g} / \mathrm{d} \text { DHA, } 1 \cdot 1 \mathrm{~g} / \mathrm{d} \\
\text { EPA) or olive oil; }\end{array}$ & $\begin{array}{l}\text { Children in the fish oil-sup- } \\
\text { plemented group attained a } \\
\text { higher score for eye and hand } \\
\text { coordination, positively with } n-3 \\
\text { and negatively with } n-6 \text {. Neutro- } \\
\text { phil production of inflammatory } \\
\text { LTB4 was inversely related to } \\
n-3 \text { LC-PUFAs intake. LTB5 } \\
\text { levels were positively correlated } \\
\text { with } n-3 \text {, particularly EPA, and } \\
\text { negatively with } n-6\end{array}$ & None & $\begin{array}{l}\text { Dunstan et al. }{ }^{(65)} \\
\quad \text { Prescott et al. }{ }^{(67)}\end{array}$ \\
\hline & 388 adults (men and women) & $\begin{array}{l}\text { German study centres } \\
\text { within the European } \\
\text { Community Respiratory } \\
\text { Health Study II }\end{array}$ & $\begin{array}{l}\text { Assessment of fish and seafood, } \\
\text { and } n \text { - } 3 \text { LC-PUFAs intake by } \\
\text { food frequency questionnaire }\end{array}$ & $\begin{array}{l}\text { Adult females with a high fish and } \\
\text { DHA intake showed a lower rate } \\
\text { of allergic sensitisation }\end{array}$ & None & Schnappinger et al. ${ }^{(66)}$ \\
\hline & $\begin{array}{l}121 \text { Lactating mothers of } 143 \\
\text { preterm infants born of } \\
<33 \text { wk gestation }\end{array}$ & $\begin{array}{l}\text { Randomized double-blind } \\
\text { placebo controlled trial }\end{array}$ & $\begin{array}{l}\text { Intervention from enrolment to } \\
\text { delivery: } \\
\text { 1) } 6 \text { capsules/d tuna oil ( } 900 \mathrm{mg} \\
\text { DHA, } 195 \mathrm{mg} \text { EPA, } 54 \mathrm{mg} \mathrm{AA}) \\
\text { 2) } 6 \text { capsules/d soy oil ( } 1.6 \mathrm{~g} \text { LA, } \\
177 \mathrm{mg} \text { ALA); }\end{array}$ & $\begin{array}{l}\text { DHA was higher in milk of sup- } \\
\text { plemented mothers }\end{array}$ & None & Smithers et al. ${ }^{(68)}$ \\
\hline & $\begin{array}{l}145 \text { pregnant women, affected } \\
\text { by allergy themselves or } \\
\text { having a husband or pre- } \\
\text { vious child with allergies }\end{array}$ & $\begin{array}{l}\text { Randomized placebo con- } \\
\text { trolled trial }\end{array}$ & $\begin{array}{l}\text { Intervention from } 25 \text { wk gestation } \\
\text { to average } 3-4 \text { mo breast-feed- } \\
\text { ing: } 1.6 \mathrm{~g} / \text { EPA and } 1.1 \mathrm{~g} / \mathrm{d} \text { DHA } \\
\text { or placebo }\end{array}$ & $\begin{array}{l}\text { The period prevalence of food } \\
\text { allergy was lower in the omega- } \\
3 \text { group, as well as incidence of } \\
\text { lgE-associated eczema }\end{array}$ & None & Furuhjelm et al..$^{(69)}$ \\
\hline & 83 healthy infants & $\begin{array}{l}2 \times 2 \text { intervention random- } \\
\text { ized controlled trial }\end{array}$ & $\begin{array}{l}\text { Intervention from } 9 \text { to } 12 \mathrm{mo} \text { : } \\
3.4 \pm 1 \cdot 1 \mathrm{~mL} / \mathrm{d} \text { and cow's milk } \\
\text { or infant formula }\end{array}$ & $\begin{array}{l}\text { Irrespective of gender, there was } \\
\text { a positive association between } \\
\text { the } 9-12-\text { mo changes in RR } \\
\text { interval and erythrocyte } n-3 \text { LC- } \\
\text { PUFAs }\end{array}$ & None & Lauritzen et al..$^{(70)}$ \\
\hline & $\begin{array}{l}24-28 \text { young adults }(18-30 \mathrm{y}) \\
\text { and } 24-28 \text { elderly }(>65 \mathrm{y})\end{array}$ & $\begin{array}{l}\text { Randomized controlled } \\
\text { trial }\end{array}$ & $\begin{array}{l}\text { 1) } 680 \mathrm{mg} / \mathrm{d} \text { DHA and } 323 \mathrm{mg} / \mathrm{d} \\
\text { EPA;3 wk } \\
\text { 2) } 1480 \mathrm{mg} / \mathrm{d} \text { EPA and } 250 \mathrm{mg} / \mathrm{d} \\
\text { DHA; } 6 \mathrm{wk}\end{array}$ & $\begin{array}{l}\text { The EPA-predominant supplement } \\
\text { raised DHA only in the young, } \\
\text { and the DHA-predominant sup- } \\
\text { plement raised EPA more in the } \\
\text { young }\end{array}$ & None & Fortier et al. ${ }^{(71)}$ \\
\hline & $\begin{array}{l}302 \text { cognitively healthy elderly } \\
\text { participants (>65y) }\end{array}$ & $\begin{array}{l}\text { 26-wk randomized double- } \\
\text { blind, placebo-controlled } \\
\text { trial }\end{array}$ & $\begin{array}{l}1800 \mathrm{mg} / \mathrm{d} \text { EPA-DHA, } 400 \mathrm{mg} / \mathrm{d} \\
\text { EPA-DHA, or placebo capsules }\end{array}$ & $\begin{array}{l}\text { There was not overall effect of } 26 \\
\text { weeks of EPA and DHA sup- } \\
\text { plementation on cognitive per- } \\
\text { formance, nor the quality of life } \\
\text { of healthy older individuals, } \\
\text { neither mental well-being }\end{array}$ & None & $\begin{array}{l}\text { Van de Rest } \\
\text { et al. } .72,73,76)\end{array}$ \\
\hline & $\begin{array}{l}1864 \text { subjects ( } 809 \text { men and } \\
1055 \text { women }\end{array}$ & $\begin{array}{l}\text { 8-y follow-up survey of the } \\
\text { SU.VI.MAX Study } \\
\text { (1994-2002) }\end{array}$ & $\begin{array}{l}\text { Assessment of fish and seafood, } \\
\text { and } n-3 \text { LC-PUFAs intake by } \\
\text { means of six 24-h recalls }\end{array}$ & $\begin{array}{l}\text { Fatty fish or } n-3 \text { LC-PUFAs con- } \\
\text { sumption higher than } 0 \cdot 10 \% \text { of } \\
\text { energy intake had lesser risk of } \\
\text { depressive episode and of } \\
\text { recurrent depressive episodes, } \\
\text { but not of single depressive epi- } \\
\text { sode. It was stronger in men } \\
\text { and in non-smokers. Smokers } \\
\text { eating fatty fish had an } \\
\text { increased risk of recurrent } \\
\text { depression }\end{array}$ & None & Astorg et al. ${ }^{(74)}$ \\
\hline
\end{tabular}


Table 1. Continued

\begin{tabular}{|c|c|c|c|c|c|c|}
\hline \multirow{2}{*}{$\begin{array}{l}\text { Dietary } \\
\text { source }\end{array}$} & \multirow[b]{2}{*}{ Participants } & \multirow[b]{2}{*}{ Design } & \multirow{2}{*}{$\begin{array}{l}n-3 \text { LC-PUFAs intervention } \\
\text { and doses }\end{array}$} & \multicolumn{2}{|c|}{ Outcomes } & \multirow[b]{2}{*}{ Reference } \\
\hline & & & & Health benefits & Health risks & \\
\hline & $\begin{array}{l}105 \text { participants }>65 \mathrm{y} \text { with } \\
\text { neovascular age-related } \\
\text { macular degeneration }\end{array}$ & $\begin{array}{l}\text { Cross-sectional popu- } \\
\text { lation-based EUREYE } \\
\text { study }\end{array}$ & $\begin{array}{l}\text { Assessment of fish and seafood, } \\
\text { and } n \text { - } 3 \text { LC-PUFAs intake by } \\
\text { food frequency questionnaire }\end{array}$ & $\begin{array}{l}\text { Eating oily fish at least once per } \\
\text { week is associated with a } \\
\text { reduction of the neovascular } \\
\text { age-related macular degener- } \\
\text { ation }\end{array}$ & None & Augood et al. ${ }^{(77)}$ \\
\hline & $\begin{array}{l}63 \text { overweight treated hyper- } \\
\text { tensive subjects }\end{array}$ & $\begin{array}{l}\text { 16-wk randomized con- } \\
\text { trolled trial }\end{array}$ & $\begin{array}{l}\text { Daily fish meal }(3.65 \mathrm{~g} / \mathrm{d} n-3 \mathrm{LC}- \\
\text { PUFAs)in a weight-loss regimen } \\
\text { or control group }\end{array}$ & $\begin{array}{l}\text { Incorporating a daily fish meal into } \\
\text { a weight-loss regimen was } \\
\text { more effective than either } \\
\text { measure alone at improving glu- } \\
\text { cose-insulin metabolism and } \\
\text { dyslipidemia }\end{array}$ & None & Mori et al..$^{(78)}$ \\
\hline & $\begin{array}{l}233 \text { health subjects }(31 \pm 5 \mathrm{y} \\
\left.\text { BMI } 28.3 \pm 1.5 \mathrm{~kg} / \mathrm{m}^{2}\right)\end{array}$ & $\begin{array}{l}\text { 8-wk randomized con- } \\
\text { trolled trial }\end{array}$ & $\begin{array}{l}\text { 1) }<260 \mathrm{mg} / \mathrm{d} n-3 \text { LC-PUFAs } \\
(n 112) \\
2)>1300 \mathrm{mg} / \mathrm{d} n-3 \text { LC- } \\
\text { PUFAs }(n 121)\end{array}$ & $\begin{array}{l}n \text {-3 LC-PUFAs group modulates } \\
\text { postprandial satiety in over- } \\
\text { weight and obese volunteers } \\
\text { during weight loss }\end{array}$ & None & Parra et al. ${ }^{(80)}$ \\
\hline & $\begin{array}{l}26 \text { overweight or moderately } \\
\left.\text { obese (BMl } 28-33 \mathrm{~kg} / \mathrm{m}^{2}\right) \\
\text { men and women }\end{array}$ & $\begin{array}{l}\text { Randomized controlled } \\
\text { trial }\end{array}$ & $\begin{array}{l}2 \text { wk control diet }(0 \% \text { fish oil, } \\
0.5 \% \text { ALA) plus } 2 \text { wk } n-3 \text { LC- } \\
\text { PUFAs diet ( } 1.4 \% \text { energy in the } \\
\text { form of EPA, DPA, and DHA } \\
\text { from fish oil, and } 2.2 \% \text { ALA } \\
\text { from plant oil) }\end{array}$ & $\begin{array}{l}\text { Dietary } n \text {-3-PUFA do not play an } \\
\text { important role in the regulation } \\
\text { of food intake, energy expendi- } \\
\text { ture, or body weight in humans }\end{array}$ & None & Kratz et al. ${ }^{(81)}$ \\
\hline & $\begin{array}{l}13 \text { patients ( } 11 \text { female, } 2 \\
\text { male, } 21-81 \text { y) with poly- } \\
\text { morphic light eruption }\end{array}$ & $\begin{array}{l}\text { 3-mo randomized con- } \\
\text { trolled trial }\end{array}$ & $\begin{array}{l}5 \text { capsules twice daily ( } 1 \mathrm{~g} \text { fish oil, } \\
18 \% \mathrm{EPA}, 12 \% \mathrm{DHA} \text { ), }\end{array}$ & $\begin{array}{l}\text { Reduction of UV-induced inflam- } \\
\text { mation by dietary fish oil }\end{array}$ & None & Rhodes et al. ${ }^{(82)}$ \\
\hline & $\begin{array}{l}51 \text { HIV-infected patients trea- } \\
\text { ted with antiretroviral } \\
\text { therapy }\end{array}$ & $\begin{array}{l}\text { 12-wk randomized double- } \\
\text { blind, placebo-controlled } \\
\text { trial }\end{array}$ & $\begin{array}{l}2 \text { capsules of Omacor twice daily } \\
\text { or } 2 \text { capsules }(n 26) \text { of placebo } \\
(n 25)\end{array}$ & $\begin{array}{l}\text { Fish oil } n-3 \text { LC-PUFAs slightly } \\
\text { decreased plasma triglycerides } \\
\text { and induced anti-inflammatory } \\
\text { effects by increasing formation } \\
\text { of anti-inflammatory LTB5 }\end{array}$ & None & Thusgaard et al. ${ }^{(83)}$ \\
\hline & 310671 women $(25-70 y)$ & $\begin{array}{l}\text { 6.4 y follow-up of the Euro- } \\
\text { pean Prospective Inves- } \\
\text { tigation Into Cancer and } \\
\text { Nutrition (1992-1998) }\end{array}$ & $\begin{array}{l}\text { Assessment of breast cancer and } \\
\text { fish and seafood, and } n-3 \text { LC- } \\
\text { PUFAs intake by food fre- } \\
\text { quency questionnaire }\end{array}$ & $\begin{array}{l}\text { No associations with breast can- } \\
\text { cer risk were observed }\end{array}$ & None & Engeset et al. ${ }^{(84)}$ \\
\hline & $\begin{array}{l}40 \text { patients with stage III non- } \\
\text { small cell lung cancer }\end{array}$ & $\begin{array}{l}\text { 4-wk randomized double- } \\
\text { blind, placebo-controlled } \\
\text { trial }\end{array}$ & $\begin{array}{l}2 \text { cans/d of protein energy-dense } \\
\text { oral nutritional supplement } \\
\text { ( } 2.0 \mathrm{~g} \mathrm{EPA}+0.9 \mathrm{~g} \mathrm{DHA} / \mathrm{d}) \text { or an } \\
\text { isocaloric control supplement }\end{array}$ & $\begin{array}{l}\text { Fish oil } n \text {-3 LC-PUFAs supplement } \\
\text { has immune-modulating effects } \\
\text { and improve nutritional status in } \\
\text { patients with non-small cell lung } \\
\text { cancer }\end{array}$ & None & Van der Meij et al. ${ }^{(85)}$ \\
\hline & $\begin{array}{l}12 \text { patients with advanced } \\
\text { lung cancer }\end{array}$ & $\begin{array}{l}\text { 6-wk randomized double- } \\
\text { blind, placebo-controlled } \\
\text { trial }\end{array}$ & $\begin{array}{l}2 \mathrm{~g} / \mathrm{d} \text { capsules (fish oil, fish oil }+ \\
\text { placebo, or fish oil plus cele- } \\
\text { coxib) }\end{array}$ & $\begin{array}{l}\text { patients receiving fish oil + } \\
\text { placebo or fish oil + celecoxib } \\
\text { showed more appetite, less fati- } \\
\text { gue, and lower C-reactive pro- } \\
\text { tein, improved body weight and } \\
\text { muscle strength }\end{array}$ & None & Cerchietti et al. ${ }^{(86)}$ \\
\hline & $\begin{array}{l}20 \text { patients with pancreatic } \\
\text { cancer }\end{array}$ & $\begin{array}{l}\text { 3-wk randomized double- } \\
\text { blind, placebo-controlled } \\
\text { trial }\end{array}$ & $\begin{array}{l}\text { Two cans (each can: } 310 \mathrm{kcal}, \\
16 \cdot 1 \mathrm{~g} \text { protein, } 1.09 \mathrm{~g} \text { EPA) of a } \\
\text { fish oil-enriched supplement per } \\
\text { day in addition to their normal } \\
\text { food intake }\end{array}$ & $\begin{array}{l}\text { Performance status, weight-gain, } \\
\text { and appetite were significantly } \\
\text { improved at } 3 \text { wk }\end{array}$ & None & Barber et al. ${ }^{(87)}$ \\
\hline & $\begin{array}{l}47866 \text { US men aged } 40-75 y \\
\text { with no cancer history in } \\
1986\end{array}$ & $\begin{array}{l}14 \mathrm{y} \text { follow-up of Health } \\
\text { Professionals Follow-Up } \\
\text { Study (1986-2000) }\end{array}$ & $\begin{array}{l}\text { Assessment of fish and seafood, } \\
\text { and } n-3 \text { LC-PUFAs intake in } \\
1986,1990, \text { and } 1994 \text { by using } \\
\text { a } 131 \text {-item semiquantitative } \\
\text { food-frequency questionnaire }\end{array}$ & $\begin{array}{l}\text { Increased dietary intakes of ALA } \\
\text { may increase the risk of } \\
\text { advanced prostate cancer. EPA } \\
\text { and DHA intakes may reduce } \\
\text { the risk of total and advanced } \\
\text { prostate cancer }\end{array}$ & None & Leitzmann et al. ${ }^{(88)}$ \\
\hline
\end{tabular}




\begin{tabular}{clll}
$\begin{array}{c}\text { Dietary } \\
\text { source }\end{array}$ & Participants & Design & $\begin{array}{l}n-3 \text { LC-PUFAs intervention } \\
\text { and doses }\end{array}$ \\
\hline $\begin{array}{c}16 \text { commonly consumed fish } \\
\text { species }\end{array}$ & $\begin{array}{c}\text { MeHg and } n \text {-3 LC-PUFAs } \\
\text { content of fish species }\end{array}$ & $\begin{array}{c}\text { Dose-response relationships for } \\
\text { MeHg and omega-3 FA effects } \\
\text { on coronary heart disease and } \\
\text { neurodevelopment }\end{array}$
\end{tabular}

Outcomes

\begin{tabular}{l}
\hline Health benefits \\
$n-3$ LC-PUFAs benefits out- \\
weighed cardiovascular and \\
neurodevelopmental MeHg risks \\
for some species (e.g., farmed \\
salmon, herring, trout). Other \\
species were associated with a \\
small net benefit (e.g., flounder, \\
canned light tuna)
\end{tabular}

Health risks

Reference

MeHg risks outweighed

Ginsberg et al. ${ }^{(96)}$

neurodevelopment

cardiovascular and ne

LC-PUFAs benefits for

some species (e.g.,

swordfish, shark). Other

species were associated

with a small net risk

(e.g., canned white

tuna, halibut)

88 healthy non-smoking men and premenopausal women (33y)

79 healthy non-smoking men and premenopausal women $(19-45 \mathrm{y})$

62 men $(>40 y)$

59 healthy male prisoners

62 men and post-menopausal women $(44-75 y)$ with hypercholesterolemia

199 menopausal women (49-65y)

1891 cases with first nonfatal $\mathrm{Ml}$ and 1891 populationbased controls; matching for age, sex, and area of resi-

6-wk double-blind, paralle

12-wk parallel randomized 2-wk double-blind, parallel randomized controlled trial

Sunflower oil (placebo), flaxseed oil (1 $\mathrm{g} / \mathrm{d}$ ALA), hempseed oil $(0.3 \mathrm{~g} / \mathrm{d}$ ALA), or fish oil $(0.6 \mathrm{~g} / \mathrm{d}$ EPA + DHA) randomized controlled trial

$\operatorname{ALA}(3.4 \mathrm{~g} / \mathrm{d})$, EPA $(2.2 \mathrm{~g} / \mathrm{d})$, or DHA (2.3 g/d) via enriched margarines controlled trial

Different doses of flax oil, fish oil, and sunflower oil in capsules; ALA doses were $1.2 \mathrm{~g} / \mathrm{d}$ $2.4 \mathrm{~g} / \mathrm{d}$, and $3.6 \mathrm{~g} / \mathrm{d}$

12-wk single-blind study

Diet with $3 \cdot 2 \mathrm{~g} / \mathrm{d}$ extra ALA

10-wk blind, parallel randomized controlled trial

Low-fat diet with extra flaxseed or with wheat bran (control); ALA dose $(3.4 \mathrm{~g} / \mathrm{d})$

$40 \mathrm{~g} / \mathrm{d}$ flaxseed or wheat germ: ALA $8.8 \mathrm{~g} / \mathrm{d}$

Case-control study

Assessment of ALA intake from FFQ
Plasma ALA levels increased after

6 wk; no differences in total

cholesterol, LDL-C, HDL-chol.,

TAG, LDL oxidation, platelet

aggregation, or inflammation

markers (CRP, TNF- $\alpha$ )

LDL and -ALA levels increased;

fasting serum TAG decreased;

no differences in total choles-

terol, LDL-chol., or HDL-chol.
2.4 and $3.6 \mathrm{~g} / \mathrm{d}$ of ALA significantly

2.4 and $3.6 \mathrm{~g} / \mathrm{d}$ of ALA significantly
increased erythrocyte ALA and

increased erythrocyte ALA and

EPA levels; no differences in
inflammation markers (CRP,

TNF- $\alpha$, sVCAM-1), total choles-

TNF- $\alpha$, sVCAM-1), total ch,
terol, TAG, or HDL-chol.

terol, TAG, or HDL-chol.
No effect on waist circumference,

No effect on waist circumferent, BMI, systolic blood
weight

weight, BMI, systolic blood
pressure; diastolic blood press-

pressure; diastolic blood press-
ure decreased and HDL-chol.

increased in non-smokers

Serum ALA levels increased; LDL-

$C$ decreased after 5 wk but not

after $10 \mathrm{wk}$, lipoprotein(A)

decreased and insulin sensitivity

(HOMA IR index) inproved,

hs-CRP) or oxidative stress

(ox-LDL urinary isoprostane)

HDL-chol. decreased

Serum ALA levels increased; mod-

est effects on apolipoproteins

$A-I$ and $B$; no effects on $L D$

electrophoretic characteristics

or markers of hemostasis and

inflammation

Inverse association between $\mathrm{MI}$

and ALA intake
None

None

None

Barceló-Coblijn
et al. ${ }^{(125)}$

None

None

None
Dodin et al. ${ }^{(128)}$

Kaul et al..$^{(123)}$

Egert et al. ${ }^{(124)}$

Sioen et al. ${ }^{(126)}$

Bloedon et al..$^{(127)}$

Campos et al. ${ }^{(129)}$ 
Table 1. Continued

\begin{tabular}{|c|c|c|c|c|c|c|}
\hline \multirow{2}{*}{$\begin{array}{l}\text { Dietary } \\
\text { source }\end{array}$} & \multirow[b]{2}{*}{ Participants } & \multirow[b]{2}{*}{ Design } & \multirow{2}{*}{$\begin{array}{l}n-3 \text { LC-PUFAs intervention } \\
\text { and doses }\end{array}$} & \multicolumn{2}{|c|}{ Outcomes } & \multirow[b]{2}{*}{ Reference } \\
\hline & & & & Health benefits & Health risks & \\
\hline & $\begin{array}{l}3575 \text { white men and women } \\
(45-64 \mathrm{y})\end{array}$ & $\begin{array}{l}\text { 14.3y follow-up of Athero- } \\
\text { sclerosis Risk in Com- } \\
\text { munities Study } \\
\text { (prospective cohort } \\
\text { study) }\end{array}$ & $\begin{array}{l}\text { Association of plasma ALA with } \\
\text { incident heart failure }\end{array}$ & $\begin{array}{l}\text { ALA status was not associated } \\
\text { with incident heart failure }\end{array}$ & None & Yamagishi et al. ${ }^{(130)}$ \\
\hline & 2009 men (50y) & $\begin{array}{l}30 \cdot 7 \mathrm{y} \text { follow-up of Uppsala } \\
\text { Longitudinal Study of } \\
\text { Adult Men (prospective } \\
\text { cohort study) }\end{array}$ & $\begin{array}{l}\text { Association of ALA in serum } \\
\text { cholesterol esters with CVD } \\
\text { mortality }\end{array}$ & $\begin{array}{l}\text { Multivariable-adjusted hazard ratio } \\
\text { was } 1 \cdot 10(1.00-1.21) \text { per } 1-\mathrm{SD} \\
\text { increase in serum ALA }\end{array}$ & None & Warensjö et al. ${ }^{(131)}$ \\
\hline & $\begin{array}{l}40 \text { cases of ischemic stroke, } \\
40 \text { cases of hemorrhagic } \\
\text { stroke and } 40 \text { healthy con- } \\
\text { trols; matching for age and } \\
\text { sex }\end{array}$ & Case-control study & $\begin{array}{l}\text { Association of ALA in erythrocytes } \\
\text { with risk of ischemic and hemor- } \\
\text { rhagic stroke }\end{array}$ & $\begin{array}{l}\text { Erythrocyte ALA concentrations in } \\
\text { hemorrhagic stroke patients and } \\
\text { ischemic stroke patients were } \\
\text { not significantly different from } \\
\text { controls; inverse association of } \\
\text { ALA with ischemic stroke }\end{array}$ & None & Park et al. ${ }^{(132)}$ \\
\hline & 2174 men $(42-60 y)$ & $\begin{array}{l}\text { 17.7 y of follow-up of Kuo- } \\
\text { pio Ischemic Heart Dis- } \\
\text { ease Risk Factor Study } \\
\text { (prospective cohort } \\
\text { study) }\end{array}$ & $\begin{array}{l}\text { Association of serum ALA with } \\
\text { incident atrial fibrillation }\end{array}$ & $\begin{array}{l}\text { Multivariable-adjusted hazard ratio } \\
\text { for serum ALA (compared to } \\
\text { Q1) was Q2: } 1.26(95 \% \mathrm{Cl} \\
0.84-1 \cdot 89), \mathrm{Q} 3: 0.74(0.46- \\
1 \cdot 20) \text {, and Q4: } 1 \cdot 14(0.7-1.79)\end{array}$ & None & Virtanen et al. ${ }^{(133)}$ \\
\hline & $\begin{array}{l}265 \text { out-of hospital sudden } \\
\text { cardiac arrest patients and } \\
415 \text { community members; } \\
\text { matching for age, sex, and } \\
\text { calendar year }\end{array}$ & Case-control study & $\begin{array}{l}\text { Association of ALA in erythrocytes } \\
\text { with risk of sudden cardiac } \\
\text { death }\end{array}$ & $\begin{array}{l}\text { Multivariable-adjusted OR over } \\
\text { quartiles of ALA in erythrocytes } \\
\text { (compared to Q1): Q2 was } 1.7 \\
\text { (95\% CI, 1.0-3.0), Q3 was } 1.9 \\
\text { (1.1-3.3), Q4 was } 2.5(1.3- \\
4.8) ; \text { association independent of } \\
\text { erythrocyte levels of EPA and } \\
\text { DHA, linoleic acid, and trans } \\
\text { fatty acids }\end{array}$ & None & Lemaitre et al. ${ }^{(134)}$ \\
\hline & $\begin{array}{l}150 \text { moderately hyperlipidemic } \\
\text { subjects }\end{array}$ & $\begin{array}{l}\text { 6-mo randomized placebo- } \\
\text { controlled, parallel study }\end{array}$ & $\begin{array}{l}0.8 \text { or } 1.7 \mathrm{~g} \mathrm{EPA}+\mathrm{DHA} / \mathrm{d}, 4.5 \text { or } \\
9.5 \mathrm{~g} \text { ALA/d, or an } n-6 \text { PUFA } \\
\text { control (FA incorporated into } \\
25 \mathrm{~g} \text { of fat spread and } 3 \text { capsu- } \\
\text { les/d) }\end{array}$ & $\begin{array}{l}\text { Fasting or postprandial lipid, glu- } \\
\text { cose, plasma } \alpha \text {-tocopherol, anti- } \\
\text { oxidant or insulin concentrations } \\
\text { or in blood pressure was not } \\
\text { significantly different between } \\
\text { treatments. Fasting triglycerides } \\
\text { after EPA + DHA intervention } \\
\text { was lower than after ALA inter- } \\
\text { vention. Susceptibility of LDL to } \\
\text { oxidation was higher after the } \\
\text { EPA + DHA intervention. }\end{array}$ & None & Finnegan et al. ${ }^{(135)}$ \\
\hline & $\begin{array}{l}57 \text { elderly }(\geq 65 \text { y) patients } \\
\text { (19 male, } 38 \text { female) }\end{array}$ & Case-control study & $\begin{array}{l}\text { Association of ALA in erythrocytes } \\
\text { with risk for mild dementia } \\
\text { (Mini-Mental Status Examin- } \\
\text { ation) }\end{array}$ & $\begin{array}{l}\text { Multivariate-adjusted regression } \\
\text { analysis showed that a higher } \\
\text { level of ALA significantly } \\
\text { decreased the risk of mild } \\
\text { dementia after adjusting for } \\
\text { age, sex, and height }\end{array}$ & None & Malgeunsinae et al. ${ }^{(136)}$ \\
\hline
\end{tabular}




\begin{tabular}{|c|c|c|c|c|c|c|}
\hline \multirow{2}{*}{$\begin{array}{l}\text { Dietary } \\
\text { source }\end{array}$} & \multirow[b]{2}{*}{ Participants } & \multirow[b]{2}{*}{ Design } & \multirow{2}{*}{$\begin{array}{l}n-3 \text { LC-PUFAs intervention } \\
\text { and doses }\end{array}$} & \multicolumn{2}{|c|}{ Outcomes } & \multirow[b]{2}{*}{ Reference } \\
\hline & & & & Health benefits & Health risks & \\
\hline & $\begin{array}{l}23 \text { subjects }(49 \cdot 3 \pm 1 \cdot 6 \mathrm{y} ; 20 \\
\text { male, } 3 \text { female), BMI } 25- \\
35 \mathrm{~kg} / \mathrm{m}^{2}\end{array}$ & $\begin{array}{l}\text { Randomized, 3-period } \\
\text { crossover design }\end{array}$ & $\begin{array}{l}\text { Average American Diet (34\% total } \\
\text { fat, } 13 \% \text { SFA, } 13 \% \text { MUFA, } 9 \% \\
\text { PUFA (7.7\% LA, 0.8\% ALA); } \\
\text { LA Diet ( } 37 \% \text { total fat, 9 } \% \\
\text { SFA, } 12 \% \text { MUFA, 16\% PUFA } \\
\text { (12.6 } \% \text { LA, 3.6 } \% \text { ALA); } \alpha \text {-LA } \\
\text { Diet (38\% total fat, 8\% SFA, } \\
12 \% \text { MUFA, 17\% PUFA } \\
\text { (10.5\% LA, 6.5\% ALA). Wal- } \\
\text { nuts and flaxseed oil: ALA pre- } \\
\text { dominant sources. Diet periods } \\
\text { lasted } 6 \text { wk, separated by 3-wk } \\
\text { compliance break during which } \\
\text { subjects consumed their usual } \\
\text { diet. }\end{array}$ & $\begin{array}{l}\text { N-telopeptide levels were signifi- } \\
\text { cantly lower following the ALA } \\
\text { diet. There was no change in } \\
\text { levels of bone-specific alkaline } \\
\text { phosphatase across the three } \\
\text { diets. Concentrations of NTx } \\
\text { were positively correlated with } \\
\text { the proinflammatory cytokine } \\
\text { TNF- } \alpha \text { for all three diets }\end{array}$ & None & Griel et al. ${ }^{(137)}$ \\
\hline & $\begin{array}{l}34 \text { T2DM }(52.4 \pm 1.5 y) \\
\text { patients (17 male, } 17 \\
\text { female) }\end{array}$ & $\begin{array}{l}\text { 12-wk randomized con- } \\
\text { trolled trial }\end{array}$ & $\begin{array}{l}\text { Participants consumed a selection } \\
\text { of bakery products containing } \\
\text { no flax }(n 9) \text {, milled flaxseed } \\
(n 13 ; 32 \mathrm{~g} / \mathrm{d}) \text {, or flaxseed oil } \\
(n 12 ; 13 \mathrm{~g} / \mathrm{d})\end{array}$ & $\begin{array}{l}\text { Flaxseed and flaxseed oil groups } \\
\text { increased plasma phospholipid } \\
n-3 \text { LC-PUFAs, but not DHA, } \\
\text { and the flaxseed oil group had } \\
\text { more EPA and DPA in plasma } \\
\text { phospholipids than the flaxseed } \\
\text { group. All groups had similar } \\
\text { caloric intakes. Control group } \\
\text { experienced a } 4 \% \text { weight gain, } \\
\text { and both flax groups had con- } \\
\text { stant body weights }\end{array}$ & None & Taylor et al. ${ }^{(138)}$ \\
\hline & $\begin{array}{l}59 \text { middle-aged dyslipidaemic } \\
\text { men }\end{array}$ & $\begin{array}{l}\text { 12-wk randomized two- } \\
\text { group, parallel-arm con- } \\
\text { trolled trial }\end{array}$ & $\begin{array}{l}\text { Dietary supplementation with flax- } \\
\text { seed oil, rich in ALA (8g/d) }\end{array}$ & $\begin{array}{l}\text { Supplementation with ALA } \\
\text { resulted in significantly lower } \\
\text { systolic and diastolic blood } \\
\text { pressure levels }\end{array}$ & None & Paschos et al. ${ }^{(139)}$ \\
\hline & $\begin{array}{l}62 \text { men and post-menopausal } \\
\text { women with pre-study low } \\
\text { density LDL-chol. (130- } \\
200 \mathrm{mg} / \mathrm{dl})\end{array}$ & $\begin{array}{l}\text { 10-wk randomized con- } \\
\text { trolled trial }\end{array}$ & $\begin{array}{l}40 \mathrm{~g} / \mathrm{d} \text { ground flaxseed-containing } \\
\text { baked products or matching } \\
\text { wheat bran products while fol- } \\
\text { lowing a low fat, low cholesterol } \\
\text { diet }\end{array}$ & $\begin{array}{l}\text { Flaxseed was well-tolerated, and } \\
\text { increased serum levels of ALA, } \\
\text { reduced LDL-chol. at } 5 \text { wk, but } \\
\text { not at } 10 \text { wk, reduced HOMA-IR } \\
\text { index, but not affect markers of } \\
\text { inflammation (IL-6, hs-CRP) or } \\
\text { oxidative stress (ox LDL, urinary } \\
\text { isoprostanes). In men, flaxseed } \\
\text { reduced HDL-chol. concen- } \\
\text { trations at } 5 \text { and } 10 \text { wk. }\end{array}$ & None & Bloedon et al..$^{(127)}$ \\
\hline & $\begin{array}{l}35 \text { non-diabetic, dyslipidemic } \\
\text { men }(38-71 \text { y) }\end{array}$ & $\begin{array}{l}\text { 12-wk randomized con- } \\
\text { trolled trial }\end{array}$ & $\begin{array}{l}15 \mathrm{ml} / \mathrm{d} \text { of flaxseed oil rich in ALA } \\
(8.1 \mathrm{~g} ; n 18) \text {, or } 15 \mathrm{ml} / \mathrm{d} \text { saf- } \\
\text { flower oil }(11.2 \mathrm{~g} \mathrm{LA} ; n 17 \text {; con- } \\
\text { trol group) }\end{array}$ & $\begin{array}{l}\text { Plasma levels of adiponectin did } \\
\text { not change after the increase in } \\
\text { ALA flaxseed oil supplemen- } \\
\text { tation did not change body } \\
\text { mass index, serum lipid concen- } \\
\text { trations, LDL density, and TNF- } \\
\alpha \text {, and adiponectin plasma } \\
\text { levels }\end{array}$ & None & Paschos et al. ${ }^{(140)}$ \\
\hline
\end{tabular}


Table 1. Continued

\begin{tabular}{|c|c|c|c|c|c|c|}
\hline \multirow{2}{*}{$\begin{array}{l}\text { Dietary } \\
\text { source }\end{array}$} & \multirow[b]{2}{*}{ Participants } & \multirow[b]{2}{*}{ Design } & \multirow{2}{*}{$\begin{array}{l}n-3 \text { LC-PUFAs intervention } \\
\text { and doses }\end{array}$} & \multicolumn{2}{|c|}{ Outcomes } & \multirow[b]{2}{*}{ Reference } \\
\hline & & & & Health benefits & Health risks & \\
\hline & $\begin{array}{l}56 \text { participants ( } 49 \text { female, } \\
7 \text { male; } 20-70 \text { y) without } \\
\text { coronary heart disease }\end{array}$ & $\begin{array}{l}\text { 26-wk randomized double- } \\
\text { blind, placebo-controlled } \\
\text { trial }\end{array}$ & $\begin{array}{l}3 \mathrm{~g} / \mathrm{d} \text { of ALA from flaxseed oil in } \\
\text { capsules }(n 31) \text { or olive oil con- } \\
\text { taining placebo capsules }(n 25)\end{array}$ & $\begin{array}{l}\text { Changes in plasma HDL-chol., } \\
\text { LDL-chol., and triglyceride con- } \\
\text { centrations did not differ } \\
\text { between the groups. Concen- } \\
\text { tration of plasma total choles- } \\
\text { terol, and less atherogenic } \\
\text { LDL1 and LDL2 subfractions } \\
\text { were higher in the flaxseed oil } \\
\text { group }\end{array}$ & None & Harper et al. ${ }^{(141)}$ \\
\hline & $\begin{array}{l}10 \text { young }(25 \pm 3 y \text { y) health } \\
\text { adults ( } 5 \text { female, } 5 \text { male) }\end{array}$ & $\begin{array}{l}\text { 4-wk randomized } \\
\text { controlled trial }\end{array}$ & $\begin{array}{l}50 \mathrm{~g} \mathrm{flaxseed} / \mathrm{d} \text { or control } \\
\text { (no flaxseed) }\end{array}$ & $\begin{array}{l}\text { ALA was increased significantly in } \\
\text { adipose tissue, and } n-3 \text { LC-- } \\
\text { PUFAs were increased in } \\
\text { plasma lipids. Plasma LDL-chol. } \\
\text { was also reduced by up to } 8 \% \text {, } \\
\text { and total urinary lignan } \\
\text { excretion was increased more } \\
\text { than fivefold. Antioxidant vita- } \\
\text { mins and lipid hydroperoxides in } \\
\text { plasma were not significantly } \\
\text { affected by flaxseed consump- } \\
\text { tion. Bowel movements per } \\
\text { week increased by } 30 \% \text { while } \\
\text { flaxseed was consumed }\end{array}$ & None & Cunnane et al..$^{(142)}$ \\
\hline & $\begin{array}{l}150 \text { healthy men and women } \\
(25-72 y)\end{array}$ & $\begin{array}{l}\text { 6-mo randomized, double- } \\
\text { blind, placebo-controlled } \\
\text { parallel study }\end{array}$ & $\begin{array}{l}\text { Placebo (no additional } n-3 \text { LC- } \\
\text { PUFAs), } 4.5 \text { or } 9.5 \mathrm{~g} \text { ALA/d, } \\
\text { and } 0.77 \text { or } 1.7 \mathrm{~g} \text { EPA + DHA/d. } \\
\text { The } n-3 \text { LC-PUFAs were pro- } \\
\text { vided in } 25 \mathrm{~g} \text { fat spread plus } 3 \\
\text { oil capsules. }\end{array}$ & $\begin{array}{l}\text { An intake of } \leq 9.5 \mathrm{~g} \mathrm{ALA} / \mathrm{d} \text { or } \\
\leq 1.7 \mathrm{~g} \text { EPA }+ \text { DHA/d does not } \\
\text { alter the functional activity of } \\
\text { neutrophils, monocytes, or lym- } \\
\text { phocytes, but it changes the } \\
\text { fatty acid composition of mono- } \\
\text { nuclear cells }\end{array}$ & None & Kew et al. ${ }^{(143)}$ \\
\hline & $\begin{array}{l}244 \text { healthy term infants (12- } \\
16 \mathrm{~d} \text {; minimum birth weight } \\
\text { of } 2500 \mathrm{~g} \text { ) }\end{array}$ & $\begin{array}{l}\text { Randomized, double-blind, } \\
\text { placebo-controlled par- } \\
\text { allel study }\end{array}$ & $\begin{array}{l}\text { Infants received study formulas } \\
\text { from } 14 \text { to } 120 \text { days of age: } \\
\text { Control (soy-based formula } \\
\text { without supplementation); } \\
\text { DHA + ARA (soy-based formula } \\
\text { supplemented with minimum } \\
\text { birth weight of } 2500 \mathrm{~g}, 17 \mathrm{mg} \\
\text { DHA } 100 \mathrm{kcal} \text { from algal oil } \\
\text { and } 34 \mathrm{mg} \text { ARA } 100 \mathrm{kcal} \text { from } \\
\text { fungal oil) }\end{array}$ & $\begin{array}{l}\text { Percentages of DHA and ARA in } \\
\text { total RBC and plasma phospho- } \\
\text { lipids were significantly higher in } \\
\text { infants in the DHA + ARA group } \\
\text { at } 120 \mathrm{~d} \text { of age. Growth rates } \\
\text { did not differ significantly } \\
\text { between feeding groups. Both } \\
\text { formulas supported normal } \\
\text { growth and were well tolerated }\end{array}$ & None & Hoffman et al. ${ }^{(144)}$ \\
\hline & $\begin{array}{l}48 \text { young subjects ( } 13 \text { male, } \\
35 \text { female) }\end{array}$ & $\begin{array}{l}\text { Randomized, double-blind, } \\
\text { placebo-controlled } \\
\text { parallel study }\end{array}$ & $\begin{array}{l}\text { 2-wk wash-in diet rich in MUFA } \\
\text { ( } 21 \% \text { energy) followed by } 3 \text {-wk } \\
\text { experimental } n-3 \text { LC-PUFAs } \\
\text { enriched diets with about } 1 \% \text { of } \\
\text { energy of ALA, EPA or DHA. } \\
n-3 \text { LC-PUFAs were provided } \\
\text { with special rapeseed oils and } \\
\text { margarines }\end{array}$ & $\begin{array}{l}\text { Dietary intake of ALA, EPA or } \\
\text { DHA led to a significant enrich- } \\
\text { ment of the respective fatty acid } \\
\text { in the LDL particles, with dietary } \\
\text { EPA preferentially incorporated. } \\
\text { ALA enrichment did not } \\
\text { enhance LDL oxidizability }\end{array}$ & None & Egert et al. ${ }^{(145)}$ \\
\hline
\end{tabular}




\begin{tabular}{|c|c|c|c|c|c|c|}
\hline \multirow{2}{*}{$\begin{array}{l}\text { Dietary } \\
\text { source }\end{array}$} & \multirow[b]{2}{*}{ Participants } & \multirow[b]{2}{*}{ Design } & \multirow{2}{*}{$\begin{array}{l}n-3 \text { LC-PUFAs intervention } \\
\text { and doses }\end{array}$} & \multicolumn{2}{|c|}{ Outcomes } & \multirow[b]{2}{*}{ Reference } \\
\hline & & & & Health benefits & Health risks & \\
\hline \multicolumn{7}{|c|}{ Enriched dairy products } \\
\hline & $\begin{array}{l}8 \text { normolipidaemic volunteers, } \\
\text { habitual partial skim milk } \\
\text { drinkers and non-eaters of } \\
\text { fish during the study }\end{array}$ & $\begin{array}{l}\text { 6-wk randomized } \\
\text { controlled trial }\end{array}$ & $\begin{array}{l}500 \mathrm{ml} / \mathrm{d} \text { partial skim milk, } 1 \mathrm{mo} ; \\
\text { then switched to } 500 \mathrm{ml} / \mathrm{d} \text { novel } \\
\text { commercially available milk } \\
\text { preparation, supplying } 400 \mathrm{mg} / \mathrm{d} \\
n-3 \text { LC-PUFAs ( } 300 \mathrm{mg} \text { EPA }+ \\
\text { DHA; } 15 \mathrm{mg} \text { vitamin E) }\end{array}$ & $\begin{array}{l}\text { The intake of a milk preparation } \\
\text { providing low amounts of } \\
\text { EPA + DHA to healthy individ- } \\
\text { uals led to marked increases of } \\
n-3 \text { LC-PUFAs and vitamin E in } \\
\text { plasma and in associated } \\
\text { increase in HDL-chol. and } \\
\text { decrease in triglycerides }\end{array}$ & None & Visioli et al. $^{(146)}$ \\
\hline & 30 subjects $(45-65 \mathrm{y})$ & $\begin{array}{l}\text { 8-wk randomized } \\
\text { controlled trial }\end{array}$ & $\begin{array}{l}500 \mathrm{ml} / \mathrm{d} \text { of semi-skimmed milk, } \\
4 \text { wk; and then } 500 \mathrm{ml} / \mathrm{d} \text { of } \\
\text { enriched } n-3 \mathrm{LC}-\mathrm{PUF} \text { As milk } \\
(60 \mathrm{mg} / 100 \mathrm{~mL} \text { EPA and DHA) }\end{array}$ & $\begin{array}{l}n \text {-3 LC-PUFAs enriched milk pro- } \\
\text { duced a significant decrease in } \\
\text { plasma concentration of trigly- } \\
\text { cerides, total and LDL choles- } \\
\text { terol accompanied by a } \\
\text { reduction in plasma levels of } \\
\text { homocysteine, vascular cell } \\
\text { adhesion molecule } 1 \text {, and an } \\
\text { increase in folic acid concen- } \\
\text { tration. Plasma and LDL oxid- } \\
\text { ability and vitamin E } \\
\text { concentration remained } \\
\text { unchanged }\end{array}$ & None & $\begin{array}{l}\text { Baró et al. }{ }^{(147)} \\
\text { Carrero et al. }{ }^{(148)}\end{array}$ \\
\hline & $\begin{array}{l}51 \text { patients ( } 25 \text { female, } 26 \\
\text { male) mildly hypertriacylgly- } \\
\text { cerolemic }\end{array}$ & $\begin{array}{l}\text { Randomized, double-blind, } \\
\text { placebo-controlled } \\
\text { cross-over trial }\end{array}$ & $\begin{array}{l}\text { Both groups received } 15 \text {-wk inter- } \\
\text { vention ( } 3 \mathrm{~g} / \mathrm{d} n \text {-3 LC-PUFAs) } \\
\text { and control dairy products con- } \\
\text { secutively with a 10-wk wash- } \\
\text { out phase between the two } \\
\text { treatments }\end{array}$ & $\begin{array}{l}\text { The consumption of } n-3 \text { LC- } \\
\text { PUFAs-enriched dairy products } \\
\text { resulted in a significant improve- } \\
\text { ment of cardiovascular risk } \\
\text { factors }\end{array}$ & None & Dawczynski et al. ${ }^{(149)}$ \\
\hline & $\begin{array}{l}25 \text { health subjects ( } 12 \text { male, } \\
13 \text { female, } 19-68 \mathrm{y})\end{array}$ & $\begin{array}{l}\text { Single-blind, randomized, } \\
\text { controlled crossover } \\
\text { study }\end{array}$ & $\begin{array}{l}\text { Subjects received a control ( } 33 \cdot 3 \mathrm{~g} \\
\text { of fat, with } 30 \mathrm{~g} \text { provided by the } \\
\text { test oil: palm olein and soybean } \\
\text { oil, ratio } 4: 1) \text { and a } n-3 \mathrm{LC}- \\
\text { PUFAs-rich meal ( } 23.2 \mathrm{~g} \text { of con- } \\
\text { trol oil and } 6 \cdot 8 \mathrm{~g} \text { fish oil, provid- } \\
\text { ing } 2 \cdot 0 \mathrm{~g} \text { EPA and } 2 \cdot 7 \mathrm{~g} \text { DHA, } \\
\text { equivalent to two portions of oily } \\
\text { fish) on two occasions in a ran- } \\
\text { dom order. Postprandial } \\
\text { measurements were made at } \\
30,60,90,120,180 \text { and } \\
240 \text { min }\end{array}$ & $\begin{array}{l}\text { Consumption of } n-3 \text { LC-PUFAs- } \\
\text { rich meal had an attenuating } \\
\text { effect on augmentation index } \\
\text { and stiffness index }\end{array}$ & None & Chong et al. ${ }^{(150)}$ \\
\hline & 88 children $(3-9 y)$ & $\begin{array}{l}\text { 7-mo randomized, } \\
\text { controlled trial }\end{array}$ & $\begin{array}{l}\text { Consumption of } 500 \mathrm{~mL} / \mathrm{d} \text { of a } \\
\text { PUFA enriched dairy drink } \\
(60 \% \text { olive oil, } 20 \% \text { peanut, } \\
\text { and } 20 \% \text { sunflower), containing } \\
\text { a quarter of the saturated fat } \\
\text { present in standard whole milk }\end{array}$ & $\begin{array}{l}\text { Enriched dairy drink reduce serum } \\
\text { levels of total cholesterol and } \\
\text { LDL-chol., without reducing } \\
\text { caloric intake }\end{array}$ & None & $\begin{array}{l}\text { Estévez-González } \\
\text { et al. }{ }^{(151)}\end{array}$ \\
\hline & $\begin{array}{l}31 \text { men }(30-60 \text { y) mildy hyper- } \\
\text { lipidemic and normotensive }\end{array}$ & $\begin{array}{l}\text { Randomized controlled } \\
\text { trial }\end{array}$ & $\begin{array}{l}\text { 4.5 g/d EPA plus DHA capsules } \\
(n \text { 25); control }(n 6) ; 3-w k \\
\text { baseline period plus 5-wk } \\
\text { intervention }\end{array}$ & $\begin{array}{l}\text { Changes in total cholesterol, LDL- } \\
\text { chol., apolipoprotein B, and } \\
\text { blood pressure with } n \text {-3 LC- } \\
\text { PUFAs were not significantly } \\
\text { different from changes for the } \\
\text { control diet. }\end{array}$ & None & Cobiac et al..$^{(152)}$ \\
\hline
\end{tabular}


Table 1. Continued

\begin{tabular}{|c|c|c|c|c|c|c|}
\hline \multirow{2}{*}{$\begin{array}{l}\text { Dietary } \\
\text { source }\end{array}$} & \multirow[b]{2}{*}{ Participants } & \multirow[b]{2}{*}{ Design } & \multirow{2}{*}{$\begin{array}{l}n-3 \text { LC-PUFAs intervention } \\
\text { and doses }\end{array}$} & \multicolumn{2}{|c|}{ Outcomes } & \multirow[b]{2}{*}{ Reference } \\
\hline & & & & Health benefits & Health risks & \\
\hline & $\begin{array}{l}297 \text { subjects }(25-65 \text { y) with } \\
\text { moderate CVD risk }\end{array}$ & $\begin{array}{l}\text { 1-y longitudinal, random- } \\
\text { ized, controlled, double- } \\
\text { blind intervention study }\end{array}$ & $\begin{array}{l}\text { Intervention: 1) } 500 \mathrm{~mL} / \mathrm{d} \text { of } \\
\text { enriched milk (EPA, DHA, oleic } \\
\text { acid, folic acid, and vitamins A, } \\
\mathrm{B}_{6}, \mathrm{D} \text {, and } \mathrm{E} \text { ), 2) } 500 \mathrm{~mL} / \mathrm{d} \text { of } \\
\text { skimmed milk, and 3) } 500 \mathrm{~mL} / \mathrm{d} \\
\text { of semi-skimmed milk (control } \\
\text { group) }\end{array}$ & $\begin{array}{l}\text { Consumption of enriched milk } \\
\text { increases serum folate and } \\
\text { HDL-chol., decreases plasma } \\
\text { triglycerides, total cholesterol, } \\
\text { and LDL-chol. Serum glucose, } \\
\text { homocysteine, and C-reactive } \\
\text { protein remained unchanged. }\end{array}$ & None & Fonollá et al. ${ }^{(153)}$ \\
\hline & $\begin{array}{l}60 \text { male patients }(60-67 \mathrm{y}) \\
\text { with peripheral vascular dis- } \\
\text { ease and intermittent claudi- } \\
\text { cation }\end{array}$ & $\begin{array}{l}\text { 1-y longitudinal, random- } \\
\text { ized, controlled, double- } \\
\text { blind intervention study }\end{array}$ & $\begin{array}{l}\text { Intervention: The supplement } \\
\text { group consumed } 500 \mathrm{~mL} / \mathrm{d} \text { of a } \\
\text { fortified dairy product containing } \\
E P A, D H A \text {, oleic acid, folic acid, } \\
\text { and vitamins } A, B_{6}, D \text {, and } \\
E \text {. The control group consumed } \\
500 \mathrm{~mL} / \mathrm{d} \text { of semiskimmed milk } \\
\text { with added vitamins } A \text { and } D \text {. }\end{array}$ & $\begin{array}{l}\text { Plasma concentrations of EPA, } \\
\text { DHA, oleic acid, folic acid, and } \\
\text { vitamins } \mathrm{B}_{6} \text { and } \mathrm{E} \text { increased } \\
\text { after treatment with sup- } \\
\text { plements. Plasma total choles- } \\
\text { terol and ApoB concentrations } \\
\text { decreased in the supplemented } \\
\text { group, and total homocysteine } \\
\text { decreased in those patients with } \\
\text { high initial concentrations. Walk- } \\
\text { ing distance before the onset of } \\
\text { claudication increased in the } \\
\text { supplemented group, and ankle- } \\
\text { brachial pressure index values } \\
\text { increased. }\end{array}$ & None & Carrero et al. ${ }^{(154)}$ \\
\hline & 40 male MI patients $(50-60 \mathrm{y})$ & $\begin{array}{l}\text { 1-y longitudinal, random- } \\
\text { ized, controlled, double- } \\
\text { blind intervention study }\end{array}$ & $\begin{array}{l}\text { Intervention: The supplement } \\
\text { group consumed } 500 \mathrm{~mL} / \mathrm{d} \text { of a } \\
\text { fortified dairy product containing } \\
\text { EPA, DHA, oleic acid, folic acid, } \\
\text { and vitamins } \mathrm{A}, \mathrm{B}_{6}, \mathrm{D} \text {, and } \\
\mathrm{E} \text {. The control group consumed } \\
500 \mathrm{~mL} / \mathrm{d} \text { of semiskimmed milk } \\
\text { with added vitamins } \mathrm{A} \text { and } \mathrm{D} \text {. }\end{array}$ & $\begin{array}{l}\text { Plasma concentrations of EPA, } \\
\text { DHA, oleic acid, folic acid, vita- } \\
\text { min } \mathrm{B}_{6} \text {, and vitamin } \mathrm{E} \text { increased } \\
\text { after supplementation. Plasma } \\
\text { total and LDL-chol., apolipopro- } \\
\text { tein B, and hs- CRP concen- } \\
\text { trations decreased in the } \\
\text { supplemented group, and } \\
\text { plasma total homocysteine } \\
\text { decreased in both groups. } \\
\text { There were no changes in heart } \\
\text { rate, blood pressure, or cardiac } \\
\text { electrocardiographic parameters } \\
\text { in either group }\end{array}$ & None & Carrero et al. ${ }^{(155)}$ \\
\hline & $\begin{array}{l}72 \text { patients with metabolic } \\
\text { syndrome }\end{array}$ & $\begin{array}{l}\text { 3-mo controlled and open- } \\
\text { label clinical trial, of } \\
\text { parallel design }\end{array}$ & $\begin{array}{l}\text { lintervention:500 ml/d of semi- } \\
\text { skimmed milk (control group, } \\
n 36 \text { ), and } 500 \mathrm{ml} / \mathrm{d} \text { of enriched } \\
(5.7 \mathrm{~g} \text { oleic acid, } 0.2 \mathrm{~g} \text { EPA + } \\
\text { DHA, } 150 \mu \mathrm{g} \text { folic acid and } \\
7.5 \mathrm{mg} \text { vitamin } \mathrm{E}, n 36) \text { semi- } \\
\text { skimmed milk }\end{array}$ & $\begin{array}{l}\text { EPA and DHA enriched skimmed } \\
\text { milk reduced total cholesterol, } \\
\text { LDL-chol., triglycerides, and } \\
\text { apolipoprotein B serum levels, } \\
\text { and glucose and homocysteine } \\
\text { plasma levels }\end{array}$ & None & Benito et al. ${ }^{(156)}$ \\
\hline & $\begin{array}{l}74 \text { healthy normolipidemic } \\
\text { men and women }(19-43 y)\end{array}$ & $\begin{array}{l}\text { 6-wk randomized con- } \\
\text { trolled trial }\end{array}$ & $\begin{array}{l}\text { Intervention: } 4 \cdot 4 \mathrm{~g} / \mathrm{d} \text { ALA (ALA } \\
\text { group), } 2 \cdot 2 \mathrm{~g} / \mathrm{d} \text { EPA (EPA } \\
\text { group), and } 2 \cdot 3 \mathrm{~g} / \mathrm{d} \text { DHA (DHA } \\
\text { group). Fatty acid ethyl esters } \\
\text { were incorporated into margar- } \\
\text { ines, which replaced the partici- } \\
\text { pant's normal spread }\end{array}$ & $\begin{array}{l}\text { The ALA, EPA, or DHA intake led } \\
\text { to a significant enrichment of } \\
\text { the LDL with the respective } n-3 \\
\text { LC-PUFAs. ALA, EPA, or DHA } \\
\text { intake did not affect fasting } \\
\text { serum concentrations of total } \\
\text { and LDL-cholesterol, but fasting } \\
\text { serum triglyceride concen- } \\
\text { trations significantly decreased. } \\
\text { DHA intake significantly } \\
\text { increased serum HDL choles- } \\
\text { terol, whereas no changes were } \\
\text { found with ALA or EPA intake }\end{array}$ & None & Egert et al..(124) \\
\hline
\end{tabular}

-mo controlled and open- lintervention: $500 \mathrm{ml} / \mathrm{d}$ of semilabel clinical trial, of 36), and $500 \mathrm{~m} / \mathrm{d}$ of enriched $5.7 \mathrm{~g}$ oleic acid, $0.2 \mathrm{~g}$ EPA DHA, $150 \mu \mathrm{g}$ folic acid and skimmed milk group), and $2.3 \mathrm{~g} / \mathrm{d} \mathrm{DHA} \mathrm{(DHA}$ group). Fatty acid ethyl esters were incorporated into margar- 


\begin{tabular}{|c|c|c|c|c|c|c|}
\hline \multirow{2}{*}{$\begin{array}{l}\text { Dietary } \\
\text { source }\end{array}$} & \multirow[b]{2}{*}{ Participants } & \multirow[b]{2}{*}{ Design } & \multirow{2}{*}{$\begin{array}{l}n-3 \text { LC-PUFAs intervention } \\
\text { and doses }\end{array}$} & \multicolumn{2}{|c|}{ Outcomes } & \multirow[b]{2}{*}{ Reference } \\
\hline & & & & Health benefits & Health risks & \\
\hline \multicolumn{7}{|c|}{ Animal-derived foods } \\
\hline & $\begin{array}{l}\text { Two groups of animals were } \\
\text { used in each of two separ- } \\
\text { ate trials }\end{array}$ & $\begin{array}{l}\text { Randomized controlled } \\
\text { trial }\end{array}$ & $\begin{array}{l}\text { (1) Hereford steers supplemented } \\
\text { (or not) with ground flaxseed } \\
\text { ( } 907 \mathrm{~g} / \mathrm{d}) \text { for } 71 \mathrm{~d} \text {, and }(2) \\
\text { Angus steers supplemented (or } \\
\text { not) with ground flaxseed } \\
\text { ( } 454 \mathrm{~g} / \mathrm{d} \text { for } 3 \mathrm{~d} \text { followed by } \\
907 \mathrm{~g} / \mathrm{d} \text { for } 110 \mathrm{~d} \text { ) }\end{array}$ & $\begin{array}{l}\text { For the Hereford group, flaxseed- } \\
\text { supplemented rations increased } \\
18: 3 n-3(4 \cdot 0 \text {-fold), } 20: 5 n-3 \\
\text { (1.4-fold), and } 22: 5 n-3(1.3- \\
\text { fold) mass as compared with } \\
\text { the control, and increased total } \\
n-3 \text { mass about } 1.7 \text {-fold. For the } \\
\text { Angus group, flaxseed ingestion } \\
\text { increased masses and compo- } \\
\text { sition of } n-3 \text { FA similarly to that } \\
\text { for the Herefords and doubled } \\
\text { the total } n-3 \text { FA mass. }\end{array}$ & $\begin{array}{l}\text { No adverse effects on FA } \\
\text { composition by grilling } \\
\text { steaks to an internal } \\
\text { temperature of } 64^{\circ} \mathrm{C} \text {. } \\
\mathrm{N}-3 \text { LC-PUFAS did not } \\
\text { affect gene expression }\end{array}$ & Kronberg et al. ${ }^{(158)}$ \\
\hline & $\begin{array}{l}36 \text { growing-finishing pigs, with } \\
\text { an average initial weight of } \\
24.8 \pm 2.6 \mathrm{~kg} \text { (mean } \pm \text { SD) }\end{array}$ & $\begin{array}{l}\text { Randomized controlled } \\
\text { trial }\end{array}$ & $\begin{array}{l}\text { Control diet, or one of three diets } \\
\text { containing } 50 \mathrm{~g} / \mathrm{kg} \text { fish silage } \\
\text { and different levels of fish fat } \\
(2.5,5.5 \text { or } 9.5 \mathrm{~g} / \mathrm{kg} \text { ). The diets } \\
\text { were either fed until the time of } \\
\text { slaughter, or } 60 \mathrm{~kg} \text { live weight } \\
\text { followed by the control diet }\end{array}$ & $\begin{array}{l}\text { No significant differences in } \\
\text { growth performance or carcass } \\
\text { quality were found among diets. } \\
\text { The total levels of } n-3 \mathrm{LC}- \\
\text { PUFAs were highest for the } 9.5 \\
\text { and the } 5.5 \mathrm{~g} / \mathrm{kg} \text { fish fat diets } \\
\text { when they were fed until slaugh- } \\
\text { ter. }\end{array}$ & $\begin{array}{l}\text { The diets containing } 2.5 \\
\text { and } 9.5 \mathrm{~g} / \mathrm{kg} \text { fish fat until } \\
\text { slaughter caused off-fla- } \\
\text { vour of bacon after both } \\
1 \text { and } 6 \text { mo of frozen } \\
\text { storage, and of loin } \\
\text { muscle after } 6 \text { mo frozen } \\
\text { storage }\end{array}$ & Kjos et al. ${ }^{(159)}$ \\
\hline & 600 crossbred pigs & $\begin{array}{l}\text { Randomized controlled } \\
\text { trial }\end{array}$ & $\begin{array}{l}4 \text { treatments: } 0 \% \text { tuna oil in diet } \\
\text { (T0; control), } 1 \% \text { unrefined tuna } \\
\text { oil in diet fed from } 35 \text { to } 90 \mathrm{~kg} \text { of } \\
\text { unrefined tuna oil in diet offered } \\
\text { during the early ( } 35-60 \mathrm{~kg} \text { BW; } \\
\text { T3-E) or late stage of fattening } \\
\text { (75 to } 90 \mathrm{~kg} \text { of BW; T3-L) }\end{array}$ & $\begin{array}{l}\text { Feeding tuna oil during a short } \\
\text { period at the end of fattening } \\
\text { (T3-L) or permanently during } \\
\text { fattening (T1) proved to be simi- } \\
\text { larly efficient in increasing } n-3 \\
\text { fatty acid content of lean and } \\
\text { adipose tissue (to about } 1 \cdot 6 \text {-fold } \\
\text { of TO). By contrast, only two- } \\
\text { thirds of this increase was } \\
\text { found when the same amount } \\
\text { of tuna oil had been fed exclu- } \\
\text { sively during early fattening } \\
\text { (T3-E). }\end{array}$ & $\begin{array}{l}\text { Flavour and acceptability } \\
\text { were most favourable in } \\
\text { pigs receiving tuna oil in } \\
\text { the early fattening } \\
\text { period (T3-E), whereas } \\
\text { it was less favourable } \\
\text { ( } P<0.05) \text { in those fed } \\
\text { tuna oil throughout fat- } \\
\text { tening (T1) }\end{array}$ & Jaturasitha et al. ${ }^{(160)}$ \\
\hline & 20 healthy adult volunteers & $\begin{array}{l}\text { Randomized controlled } \\
\text { trial }\end{array}$ & $\begin{array}{l}\text { Sensory quality assessment of } n-3 \\
\text { LC-PUFAs-rich functional } \\
\text { Bruehwurst sausages made } \\
\text { with a range of } n-3 \text { PUFA } \\
\text { sources }\end{array}$ & $\begin{array}{l}\text { TBARS values of the sausages } \\
\text { were low, even after storage. } \\
\text { Microbiological and physico- } \\
\text { chemical properties of the } \\
\text { sausages were generally } \\
\text { unaffected by addition of } \\
\text { omega-3 fatty acid sources. }\end{array}$ & $\begin{array}{l}\text { Some of the omega-3 fatty } \\
\text { acid sources tested } \\
\text { caused off-flavours, not } \\
\text { always described as } \\
\text { "fishy" }\end{array}$ & Muench et al. ${ }^{(161)}$ \\
\hline
\end{tabular}


Table 1. Continued

\begin{tabular}{|c|c|c|c|c|c|c|}
\hline \multirow{2}{*}{$\begin{array}{l}\text { Dietary } \\
\text { source }\end{array}$} & \multirow[b]{2}{*}{ Participants } & \multirow[b]{2}{*}{ Design } & \multirow{2}{*}{$\begin{array}{l}n \text {-3 LC-PUFAs intervention } \\
\text { and doses }\end{array}$} & \multicolumn{2}{|c|}{ Outcomes } & \multirow[b]{2}{*}{ Reference } \\
\hline & & & & Health benefits & Health risks & \\
\hline & $\begin{array}{l}\text { Eighty-eight } 40 \text {-week-old ISA } \\
\text { Brown laying hens }\end{array}$ & $\begin{array}{l}\text { Randomized controlled } \\
\text { trial }\end{array}$ & $\begin{array}{l}\text { The trial lasted for } 28 \mathrm{~d} \text { and } \\
\text { started after a pre-experimental } \\
\text { period of } 21 \mathrm{~d} \text {, where animals } \\
\text { received the same experimental } \\
\text { treatments: } 9 \text { diets: } 3 \text { levels } n-3 \\
\text { FA supplementation }(2.9,3 \cdot 7 \\
\text { and } 4.5 \mathrm{~g} / \mathrm{kg} \text { ) from } 3 \text { different } \\
\text { sources (marine algae oil, and } \\
\text { two marine fish oils rich in EPA } \\
\text { or in DHA), plus } 2 \text { diets (fixed } \\
\mathrm{CLA}(2.5 \mathrm{~g} / \mathrm{kg} \text { ) and HOSO } \\
(30 \mathrm{~g} / \mathrm{kg}) \text {. Feed and water were } \\
\text { supplied ad libitum. The hens } \\
\text { received } 15 \mathrm{~h} \text { light } / \mathrm{d} \text { throughout } \\
\text { the experiment. Room tempera- } \\
\text { ture was also controlled at } \\
\text { about } 24^{\circ} \mathrm{C} \text {. }\end{array}$ & $\begin{array}{l}\text { An increase in } n-3 \text { PUFA sup- } \\
\text { plementation had little effect on } \\
\text { proportions of CLA, MUFA, SFA } \\
\text { or total PUFA in yolk fat, but } \\
\text { increased } n-3 \text { LC-PUFAs and } \\
\text { decreased } n-6 \text { LC-PUFA. An } \\
\text { increment of dietary } n-3 \text { LC- } \\
\text { PUFAs impaired linearly egg } \\
\text { acceptability by consumers. }\end{array}$ & None & Cachaldora et al. ${ }^{(162)}$ \\
\hline & 25 healthy adult volunteers & $\begin{array}{l}\text { Randomized double-blind } \\
\text { crossover trial }\end{array}$ & $\begin{array}{l}\text { Subjects fed 3wk with } 5 \text { normal } \\
\text { eggs/wk, and next } 3 \text { wk fed with } \\
\text { enriched eggs/wk. A second } \\
\text { group received eggs in the } \\
\text { inverse sequence. Enriched } \\
\text { eggs from hens feed tuna oil } \\
5 \% \text { ( } 9 \text { times more } n-3 \text { LC- } \\
\text { PUFAs) }\end{array}$ & $\begin{array}{l}\text { Decrease in serum triglycerides. } \\
\text { No change on LDL-chol., and } \\
\text { HDL-chol. }\end{array}$ & None & Bovet et al. ${ }^{(163)}$ \\
\hline & $\begin{array}{l}126 \text { 28-week-old Warren lay- } \\
\text { ing hens with similar body } \\
\text { weight }(2 \mathrm{~kg} \text { ) and egg par- } \\
\text { ameters were randomly } \\
\text { divided into } 7 \text { groups of } 18 \\
\text { birds each ( } 6 \text { replica- } \\
\text { tes/group) }\end{array}$ & $\begin{array}{l}\text { 6-wk randomized con- } \\
\text { trolled trial }\end{array}$ & $\begin{array}{l}\text { Hens received one of these diets } \\
\text { (all were isoenergetic): Control } \\
\text { (complete diet); control contain- } \\
\text { ing } 10 \mathrm{~g} / \mathrm{kg} \text { linseed oil; control } \\
\text { containing } 49.5 \mathrm{~g} / \mathrm{kg} \text { fish oil; } \\
\text { control supplemented with } \\
16.7 \mathrm{~g} / \mathrm{kg} \text { DHA-rich Schizochy- } \\
\text { trium sp.microalgae; control } \\
\text { supplemented further sup- } \\
\text { plemented with } 5.5 \mathrm{mg} / \mathrm{kg} \text { pot- } \\
\text { assium iodide; control } \\
\text { supplemented further sup- } \\
\text { plemented with } 2.03 \mathrm{mg} / \mathrm{kg} \\
\text { sodium selenite; control sup- } \\
\text { plemented further supplemented } \\
\text { with } 5.5 \mathrm{mg} / \mathrm{kg} \text { potassium iodide } \\
\text { and } 2.03 \mathrm{mg} / \mathrm{kg} \text { sodium selenite. }\end{array}$ & $\begin{array}{l}\text { Improvement in egg weight and in } \\
\text { the DHA content of yolks by } \\
\text { feeding hens a microalgae-rich } \\
\text { diet, which avoids the unplea- } \\
\text { sant flavours associated with } \\
\text { fish oil supplementation }\end{array}$ & None & Rizzi et al. ${ }^{(164)}$ \\
\hline \multirow[t]{2}{*}{ Krill oil } & $\begin{array}{l}76 \text { overweight obese men } \\
\text { and women }\end{array}$ & $\begin{array}{l}\text { 4-wk randomized double- } \\
\text { blind parallel arm trial }\end{array}$ & $\begin{array}{l}\text { Capsules containing } 2 \mathrm{~g} / \mathrm{d} \text { of krill } \\
\text { oil, menhaden oil, or control } \\
\text { (olive oil) }\end{array}$ & $\begin{array}{l}\text { Krill oil supplementation increased } \\
\text { plasma EPA and DHA, } \\
\text { decreased plasma urea, and } \\
\text { was well tolerated }\end{array}$ & None & Maki et al.. (167) \\
\hline & $\begin{array}{l}120 \text { patients with hyper- } \\
\text { lipidemia }\end{array}$ & $\begin{array}{l}\text { Multi-center, 3-mo, pro- } \\
\text { spective, randomized } \\
\text { study followed by a } \\
3 \text {-mo, controlled follow- } \\
\text { up of patients treated } \\
\text { with } 1 \mathrm{~g} \text { and } 1.5 \mathrm{~g} \text { krill } \\
\text { oil daily }\end{array}$ & $\begin{array}{l}\text { Four groups: 1) Krill oil at BMI- } \\
\text { dependent daily dosage } \\
2-3 \mathrm{~g} / \mathrm{d} \text {. 2) Krill oil } 1-1.5 \mathrm{~g} / \mathrm{d} \text {. 3) } \\
\text { Fish oil (180 mg EPA }+120 \mathrm{mg} \\
\text { DHA/g fish oil; } 3 \mathrm{~g} / \mathrm{d}) .4 \text { ) } \\
\text { Placebo }\end{array}$ & $\begin{array}{l}\text { Krill oil decreased total choles- } \\
\text { terol, LDL, and triglycerides, } \\
\text { and increasing HDL levels }\end{array}$ & None & Bunea et al. ${ }^{(168)}$ \\
\hline
\end{tabular}




\begin{tabular}{|c|c|c|c|c|c|c|}
\hline \multirow{2}{*}{$\begin{array}{l}\text { Dietary } \\
\text { source }\end{array}$} & \multirow[b]{2}{*}{ Participants } & \multirow[b]{2}{*}{ Design } & \multirow{2}{*}{$\begin{array}{l}n \text {-3 LC-PUFAs intervention } \\
\text { and doses }\end{array}$} & \multicolumn{2}{|c|}{ Outcomes } & \multirow[b]{2}{*}{ Reference } \\
\hline & & & & Health benefits & Health risks & \\
\hline \multirow{3}{*}{ Seal oil } & $\begin{array}{l}70 \text { adult women with pre- } \\
\text { menstrual syndrome }\end{array}$ & $\begin{array}{l}\text { 3-mo randomized double- } \\
\text { blind controlled trial }\end{array}$ & $\begin{array}{l}\text { Intervention with } 1 \mathrm{~g} / \mathrm{soft} \text { gels } \\
\text { ( } 2 \text { times/d with meals) of either } \\
\text { Neptune krill oil or fish oil }(18 \% \\
\text { EPA }+12 \% \text { DHA })\end{array}$ & $\begin{array}{l}\text { Neptune krill oil decreased dysme- } \\
\text { norrhea and emotional symp- } \\
\text { toms of premenstrual syndrome } \\
\text { and was more effective for the } \\
\text { complete management of pre- } \\
\text { menstrual symptoms compared } \\
\text { to fish oil }\end{array}$ & None & Sampalis et al. ${ }^{(169)}$ \\
\hline & $\begin{array}{l}19 \text { healthy, normocholesterole- } \\
\text { mic subjects }\end{array}$ & $\begin{array}{l}\text { 42-d randomized con- } \\
\text { trolled trial }\end{array}$ & $\begin{array}{l}20 \mathrm{~g} \text { of encapsulated seal oil } \\
\text { (EPA; DHA; DPA) or } 20 \mathrm{~g} \text { of } \\
\text { vegetable oil (control) per day }\end{array}$ & $\begin{array}{l}\text { Seal oil supplementation } \\
\text { decreased the } n-6 / n-3 \text { ratio and } \\
\text { increased EPA, DHA, and DPA } \\
\text { and the ratio of EPA/ARA and } \\
\text { DHA/ARA in the serum phos- } \\
\text { pholipid and NEFA, while exhi- } \\
\text { biting a modest beneficial effect } \\
\text { on fibrinogen and protein C } \\
\text { levels }\end{array}$ & None & Conquer et al. ${ }^{(170)}$ \\
\hline & $\begin{array}{l}144 \text { patients with nonalcoholic } \\
\text { fatty liver disease and } \\
\text { hyperlipidemia }\end{array}$ & $\begin{array}{l}\text { Randomized controlled } \\
\text { trial }\end{array}$ & $\begin{array}{l}\text { Two groups: 1) recommended diet } \\
\text { and } 2 \mathrm{~g} n \text {-3 PUFA from seal } \\
\text { oils. 2) recommended diet and } \\
2 \mathrm{~g} \text { placebo. Intervention } 3 \\
\text { times /d }\end{array}$ & $\begin{array}{l}n \text { - } 3 \text { LC-PUFAs from seal oils is } \\
\text { safe and efficacious for patients } \\
\text { with nonalcoholic fatty liver dis- } \\
\text { ease associated with hyperlipi- } \\
\text { demia and can improve their } \\
\text { total symptom scores, ALT, } \\
\text { serum lipid levels and normali- } \\
\text { zation of ultrasonographic } \\
\text { evidence }\end{array}$ & None & Zhu et al. ${ }^{(171)}$ \\
\hline
\end{tabular}

Abbreviations: ALA: $\alpha$-linolenic acid; ARA: arachidonic acid; CLA: conjugated linoleic acid; BMI: body mass index; CD69: cluster of differentiation 69; CRP: C-reactive protein; CVD: cardiovascular diseases; DHA: docosahexaenoic acid; DPA: docosapentaenoic acid; EPA: eicosapentaenoic acid; FFQ: food frequency questionnaire; HOMA-IR index: homeostasis model assessment-insulin resistence; hs-CRP: high-sensitivity C-reactive protein; HOSO: higholeic sunt lower oll, IL-6. interleukine-6, LA: linoleic acid, LTB4: Ieukot omega-3 long chain polyunsaturated fatty acids; NO: nitric oxide; RBCs: red blood cells; SFA: saturated fatty acids; sICAM- 1 : soluble cell 
Daily intake of fish oil $n$-3 LC-PUFAs for 37 months decreased $16 \%$ all causes of mortality and $24 \%$ the incidence of death due to myocardial infarction. This benefit putatively arises from the incorporation of EPA and DHA into cardiomyocyte phospholipids at the expense of ARA during high-dose fish-oil supplementation ${ }^{(18)}$. Fish oil consumption decreased tumour necrosis factor-alpha (TNF $\alpha$ ) production in healthy subjects and improves body weight in severe heart failure ${ }^{(19)}$. However, restenosis after coronary angioplasty was not reduced by supplemental fish oil ${ }^{(20)}$.

Consumption of fish has been associated with a significantly reduced progression of coronary atherosclerosis in women with coronary artery disease ${ }^{(21)}$. Atlantic salmon fillets very high in $n$-3 LC-PUFAs of marine origin seemed to impose favourable biochemical changes (reductions of serum triglycerides, vascular cell adhesion molecule-1 and interleuki $n-6)$ in patients with coronary heart disease ${ }^{(22)}$. Findings from short- and long-term randomized trials pointed out that fish $n$-3 LC-PUFAs intake are inversely related to blood pressure, either on hypertensive or nonhypertensive persons, with small estimated effect size ${ }^{(23)}$.

After 6-year follow up, the age-adjusted models showed no evidence of an association between fish consumption or omega-3 fatty acid intake and incident of atrial fibrillation $(\mathrm{AF})$ in a large sample of older, postmenopausal women (44720 participants from the Women's Health Initiative clinical trials) who were not enrolled in a dietary modification intervention arm and without $\mathrm{AF}$ at baseline ${ }^{(24)}$. Fish oil $n-3$ LCPUFAs have not a protective effect on cardiac arrhythmia. Current data neither proved nor disproved a beneficial or a detrimental effect for subgroups of patients with specific underlying pathologies ${ }^{(25)}$.

DHA and EPA rich fish-oil supplements taken with a high-fat meal preserved impairments in endothelial function ${ }^{(26)}$. There was no effect on cardiovascular biomarkers or mood in patients with ischemic stroke submitted to $12 \mathrm{wk}$ of treatment with moderate-dose fish oil supplements $(3 \mathrm{~g} / \mathrm{d}$ fish oil containing $1.2 \mathrm{~g}$ total omega-3: $0.7 \mathrm{~g}$ DHA; $0.3 \mathrm{~g} \mathrm{EPA}$ ). It is possible that insufficient dose, short duration of treatment, and/or oxidation of the fish oils may have influenced these outcomes ${ }^{(27)}$.

Beneficial effects of fish oil $n$-3 LC-PUFAs on cardiac risk factors and heart rate variability have been also found in people with epilepsy ${ }^{(28)}$. However, the administration of five fish oil capsules with every meal $(1260 \mathrm{mg} / \mathrm{d}$ EPA and $540 \mathrm{mg} / \mathrm{d}$ DHA) in healthy middle-aged Japanese men with a high level of fish consumption for 4 weeks did not demonstrate a decrease in plasma TAG, cholesterol, LDL-cholesterol, and whole-blood viscosity. Further, no changes in the fatty acid composition of plasma and erythrocyte phospholipids were noted $^{(29)}$. A progressive and significant increase in total hyperhomocysteinemia was observed after 8 weeks of dietary supplementation with $6 \mathrm{~g} / \mathrm{d}$ of fish oil. This increase was not associated with changes in plasma folate or vitamin $\mathrm{B}_{12}$ concentrations $^{(30)}$.

In comparison with corn oil, fish oil tended to increase HDL and decreased LDL concentration, and to decrease insulin sensitivity, but it has no effect on oxidized $\mathrm{LDL}^{(31)}$. Once-a-day intakes of plant sterol-enriched yoghurt drink ( $2 \mathrm{~g}$ plant sterols/d) and fish oil capsules ( $2 \mathrm{~g} / \mathrm{d}$ fish oil $n$-3 LC-PUFAs) reduced 15\% TAG and increased 5.4\% HDL-cholesterol in mildly hypercholesterolaemic $35-55 \mathrm{y}-\mathrm{O}$ adults ${ }^{(32)}$.

A 30-year follow-up survey of the Dutch and Finnish cohorts of the Seven Countries Study showed that an increase in the fish consumption was inversely related to glycaemia ${ }^{(33)}$. To take $\geq 1$ versus $<1$ portion/week of fish was associated with a lower risk of $\mathrm{T} 2 \mathrm{DM}^{(34)}$. Moreover, the risk of T2DM in an elderly population was lowered by increased fish and $n$-3 LC-PUFAs consumption ${ }^{(35)}$. However, a large epidemiological study of healthy adults showed that the relative risk of T2DM was slightly higher in women who consumed $\geq 5$ servings fish/wk than those who consumed fish $\leq 1 /$ mo, after adjustment by other dietary and lifestyle risk factors. The authors explained the results by the fact that toxins such as dioxins and methylmercury may interrupt insulin signalling pathways. The authors also hypothesized that $n$-3 LC-PUFAs may contribute to higher glucose concentrations through other mechanisms, i.e.: $n$-3 LC-PUFAs can decrease glucose utilization and increase glucagon-stimulated C-peptide, or increase hepatic gluconeogenesis ${ }^{(36)}$. Several clinical studies also reported that $n$-3 LC-PUFAs may worsen glucose tolerance and insulin resistance in T2DM patients who consumed large amounts of fish oil ${ }^{(37-40)}$. It has been pointed out that these negative effects were due to the high doses of $n$-3 LCPUFAs used, such as $\geq 10 \mathrm{~g} / \mathrm{d}$ fish oil.

A prospective study of 36328 women (mean age $54.6 \mathrm{y}$ ) who participated in the Women's Health Study (1992-2008) suggested an increased risk of T2DM with the intake of marine $n$-3 LC-PUFAs, especially with high intakes $(\geq 0 \cdot 2 \mathrm{~g}$ omega- $3 / \mathrm{d}$ or $\geq 2$ servings of fish/d) ${ }^{(41)}$. However, unfavourable associations between marine $n-3$ LC-PUFAs intake and glucose control was not found ${ }^{(42)}$. In healthy individuals a moderate supplementation of fish oil did not affect insulin sensitivity, insulin secretion, beta-cell function or glucose tolerance $^{(43)}$. Further, in a crossover study of subjects with T2DM, enrichment with fish oil $n-3$ LC-PUFAs failed to affect insulin sensitivity and secretion ${ }^{(44)}$, but another randomized crossover dietary intervention study with two 8-week periods reported that an increase in oily fish consumption increased insulin sensitivity in young iro $n$-deficient women ${ }^{(45)}$

Current evidence indicates that fish oil EPA and DHA can prevent the development of inflammatory diseases by affecting different steps of the immune response. DHA, but not EPA, suppresses $T$ lymphocyte activation ${ }^{(46)}$. The capacity of $n$-3 LC-PUFAs to modulate the synthesis of eicosanoids, activity of nuclear receptor and transcription factors, and production of resolvins, may also mitigate inflammatory processes already present. In a 8 -wk intervention trial, 324 subjects (aged 20-40 years, and BMI $27 \cdot 5-32.5 \mathrm{~kg} / \mathrm{m}^{2}$ ) that took salmon $(3 \times 150 \mathrm{~g} / \mathrm{wk}, 2 \cdot 1 \mathrm{~g} / \mathrm{d}$ LC-PUFA $)$ or $\operatorname{cod}(3 \times 150 \mathrm{~g} /$ wk, $0.3 \mathrm{~g} / \mathrm{d} n$-3 LC-PUFAs) or fish oil capsules $(1.3 \mathrm{~g} / \mathrm{d} n$-3 LC-PUFAs) showed significant decreases in inflammation parameters (high-sensitivity C-reactive protein, interleuki $n-6$, glutathione reductase, and prostaglandin $F_{2} \alpha$ ), a mechanism by which PUFAs reduce CVD, but also they experienced weight loss $(-5 \cdot 2 \pm 3 \cdot 2 \mathrm{~kg})^{(47)}$, and decreased diastolic and systolic blood pressure ${ }^{(48)}$. Similar results were obtained in 
subjects (35-70 years) after an 8-wk food-based intervention trial taking salmon, an oily fish ${ }^{(49)}$. Dietary fish oil $n$-3 LCPUFAs supplementation had a markedly protective effect in suppressing exercise-induced bronchoconstriction in elite athletes, which may be attributed to their antiinflammatory properties. Fish oil $n$-3 LC-PUFAs supplementation decreased leukotriene (LT)E4, $9 \alpha, 11 \beta$-prostaglandin F2, LTB4, TNF $\alpha$, and interleukin- $1 \beta^{(50)}$.

$n$-3 LC-PUFAs are potentially useful anti-inflammatory agents. To intake fish oil $960 \mathrm{mg} / \mathrm{d}$ of EPA and $600 \mathrm{mg} / \mathrm{d}$ of DHA can decrease C-reactive protein levels ${ }^{(51)}$. An 8-wk consumption of fatty fish decreased lipids which are potential mediators of lipid-induced insulin resistance and inflammation $^{(52)}$. Dietary $n-3$ fatty acids have been associated with lower levels of inflammation and endothelial activation, which may partially explain the effect of $n-3$ LC-PUFAs in preventing cardiovascular disease ${ }^{(53)}$.

Parenteral supplementation with fish oil n-3 LC-PUFAs emulsion decreased the magnitude and persistence time of the systemic inflammatory response syndrome (SIRS), markedly retrieve the unbalance of the pro-/anti-inflammatory cytokines, improve severe condition of illness and may provide a new way to regulate the $\operatorname{SIRS}^{(54)}$.

Fish oil $n$-3 LC-PUFAs reduced the requirement for nonsteroidal antiinflammatory drugs (NSAID) in patients with rheumatoid arthritis ${ }^{(55)}$, and are a safer alternative to NSAID for treatment of nonsurgical neck or back pain ${ }^{(56)}$. Cod liver oil supplements containing $n$-3 LC-PUFAs may be used as NSAID-sparing agents in rheumatoid arthritis patients ${ }^{(57)}$. The combination of fish oil and paracetamol suppressed $\mathrm{PGE}_{2}$ synthesis by an amount equivalent to that from maximum therapeutic doses of NSAID, and enhanced suppression of nociceptive $\mathrm{PGE}_{2}$ synthesis and thereby provided additive symptomatic benefits ${ }^{(58)}$. Asthma, another highly prevalent chronic inflammatory disease, may also positively respond to fish oil supplements ${ }^{(59)}$.

In spite of a high intake of fish oil, $n$-3 LC-PUFAs may be associated with decreased inflammation. A 12-wk randomized, double-blind placebo-controlled intervention trial in healthy subjects aged 50-70 years did not show that $3.5 \mathrm{~g} / \mathrm{d}$ fish oil ( $1.5 \mathrm{~g} / \mathrm{d} n$-3 LC-PUFAs) significantly affected the serum inflammatory response (it did not significantly affect serum concentrations of cytokines, chemokines or cell adhesion molecules), nor did patterns of inflammatory markers ${ }^{(60)}$.

Fish oil $n$-3 LC-PUFAs blunted the endocrine stress response and the increase in body temperature, but had no impact on cytokine production after endotoxin challenge, which has been shown to mimic several aspects of sepsis. These findings conflict with the postulated anti-inflammatory effects of fish oil on ARA metabolism and cytokine release. These results suggest that fish oil may exert beneficial effects in sepsis though non-inflammatory ${ }^{(61)}$. However, the use of immunonutrition including fish oil in critical ill patients or patients with severe sepsis may exert an excess mortality. All of which require further research.

A high fish oil EPA and DHA intake (1.8g EPA and DHA/d, 26 weeks) changed the expression of 1040 genes, and resulted in a decreased expression of genes involved in inflammatory- and atherogenic-related pathways, such as nuclear transcription factor kappaB signaling, eicosanoid synthesis, scavenger receptor activity, adipogenesis, and hypoxia signaling ${ }^{(62)}$.

Thirty six girls aged $18-22$ years were supplemented 3 months with $15 \mathrm{~mL}$ fish oil daily $(550 \mathrm{mg} / \mathrm{d} \mathrm{EPA} ; 205 \mathrm{mg} / \mathrm{d})$ by means a cross-over clinical trial. They reduced symptoms of dysmenorrhoea, low back pain and abdominal pain, and needed significantly fewer rescue doses of ibuprofen while using fish oil ${ }^{(63)}$

Pregnant women aged 18-41 years supplemented from week 22 with modified fish oil showed high thiobarbituric acid-reactive substances (TBARS), an oxidative stress index in lipids, at week 30, and minor changes of uric acid increased and beta-carotene as well as trolox-equivalent antioxidative capacity (TEAC) from week 20 to delivery. Fish oil $n-3$ LC-PUFAs supplementation improved infant neurological development, it causes additional increase of oxidative stress at week 30, but it also did not decrease antioxidant status during the second half of pregnancy ${ }^{(64)}$. Maternal fish oil supplementation during pregnancy $(2 \cdot 2 \mathrm{~g} / \mathrm{d}$ DHA and $1 \cdot 1 \mathrm{~g} / \mathrm{d}$ EPA from 20 weeks' gestation until delivery) was safe for the foetus and infant, and might have potentially beneficial effects on the child's eye and hand coordination ${ }^{(65)}$

Fish intake also plays a protective role in the development of allergic diseases in women because of its high $n-3$ LC-PUFAs contents. It is not understood why this association was only seen in females, but gender-related differences in metabolism of PUFA could be a possible explanation ${ }^{(66)}$. Supplementation of pregnant women with allergic disease with fish oil $(3.7 \mathrm{~g} / \mathrm{d}$ of $n-3$ LC-PUFAs) for the final 20 weeks of pregnancy decreased neutrophil LTB4 production, pro-inflammatory IL-6 responses and regulatory IL-10 responses by lipopolysaccharide-stimulated neonatal mononuclear cells, and a trend for less inflammatory products (LTB5) in neonates. It provides evidence that fish $n$-3 LC-PUFAs can influence early immune development ${ }^{(67)}$. Milk of lactating mothers supplemented with tuna oil had high DHA and ALA contents, which are important nutrients in the infant preterm diet ${ }^{(68)}$. The maximum DHA levels in human breast milk exceed $1 \%$ of total fatty acids in high-fish-consuming populations. Consumption of DHA-rich human milk as sole source of nutrition provided approximately $315 \mathrm{mg} / \mathrm{d}$ in infants $1-6$ months of age, and appeared to be a safe level of intake, without adverse events in infants. Daily maternal supplementation with either fish oil $1.6 \mathrm{~g}$ EPA and $1.1 \mathrm{~g}$ DHA or placebo in pregnant women affected by allergy themselves or having a husband or previous child with allergies from the 25 th gestational week to average 3-4 months of breastfeeding, decreased the period prevalence of food allergy, as well as the incidence of IgE-associated eczema during the first year of life in infants with a family history of allergic disease ${ }^{(69)}$. The $n$-3 LC-PUFAsstatus in late infancy affected heart rhythm in infants similar to that observed in adults, and influenced on brain development and CNS function, irrespectively of gender ${ }^{(70)}$.

Elderly people are susceptible to cardiovascular and neurological illnesses, which seem to be related in part to lower intake of $n-3$ fatty acids ${ }^{(71)}$. Furthermore, supplementation with high or low doses of fish oil $n-3$ LC-PUFAs for 26 
weeks influenced neither the cognitive performance ${ }^{(72)}$, nor the quality of life of healthy older individuals, measured by means of the WHO's quality of life questionnaire ${ }^{(73)}$.

Subjects consuming fatty fish or with an intake of $n$-3 LCPUFAs higher than $0 \cdot 10 \%$ of energy intake had a significantly low risk of depressive episode and of recurrent depressive episodes, but not of single depressive episode. These associations were stronger in men and in non-smokers, but smokers eating fatty fish had an increased risk of recurrent depression. Then, usual intake of fatty fish or $n$-3 LC-PUFAs may decrease the risk of recurrent depression in non-smokers ${ }^{(74)}$.

Few effects of $n$ - 3 LC-PUFAs on cognition and mood states, few risk-averse decisions, and improved scores on the control/perfectionism scale of the cognitive reactivity measure have been also found, but no effects on other cognitive tasks ${ }^{(75)}$. A randomized, double-blind, placebo-controlled trial did not observed effect of EPA and DHA supplementation for $26 \mathrm{wk}$ on mental well-being in older ( $\geq 65$ years) population $^{(76)}$. Eating oily fish at least once per week were associated with a reduction of neovascular age-related macular degeneration $^{(77)}$.

Incorporating a daily fish meal rich in $n$-3 LC-PUFAs into a weight-loss regimen was more effective than either measure alone at improving glucose-insulin metabolism and dyslipidemia, and also reduced cardiovascular risk ${ }^{(78)}$. Controlled trials using whole fish as a test meal were encouraged to be able to elucidate the role of different constituents of fish for human health ${ }^{(79)}$. Validated visual analogue scale assessment revealed low hunger sensations in volunteers $(31 \pm 5$ years; BMI $\left.28.3 \pm 1.5 \mathrm{~kg} / \mathrm{m}^{2}\right)$ after an intervention $(>1300 \mathrm{mg} / \mathrm{d}$ of $n-3$ LC-PUFAs) on the last $2 \mathrm{wk}$ of an 8 -wk energy-restricted balanced diet (weight loss $=-5.9 \pm 3.1 \%$ ). Therefore, $n-3$ LC-PUFAs seems to modulate postprandial satiety in overweight and obese volunteers during weight loss, and may be considered nutritional factors with a potential to modulate food intake ${ }^{(80)}$. However, a controlled randomized dietary trial showed that dietary $n-3$ LC-PUFAs do not play an important role in the regulation of food intake, energy expenditure, or body weight in humans ${ }^{(81)}$.

The sunburn response is markedly reduced by dietary fish oil rich in $n$-3 LC-PUFAs. Reduction of UV-induced inflammation by fish oil may be due, at least partially, to lowered $\mathrm{PGE}_{2}$ levels, suggesting a clinical application for fish oil $n-3$ LC-PUFAs ${ }^{(82)}$.

Treatment of antiretroviral treated HIV-infected patients with fish oil $n$-3 LC-PUFAs slightly decreased plasma TAG and induced anti-inflammatory effects by increasing formation of anti-inflammatory LTB5. No other changes were observed $^{(83)}$.

Some in vitro and animal studies have suggested an inhibitory effect of marine $n-3$ fatty acids on breast cancer growth, but no significant associations between intake of total fish and breast cancer risk were observed in 310671 women aged 25-70 years at recruitment into the European Prospective Investigation Into Cancer and Nutrition ${ }^{(84)}$. Oral nutritional supplement containing fish oil $2.0 \mathrm{~g} / \mathrm{d}$ EPA and $0.9 \mathrm{~g} / \mathrm{d}$ DHA had immune-modulating effects and could improve nutritional status in patients with non-small cell lung cancer
(NSCLC) undergoing multimodality treatment ${ }^{(85)}$. A combination of fish oil $n-3$ LC-PUFAs and cyclooxygenase-2 inhibitor decreased some of the signs and symptoms associated with a Systemic Immune-Metabolic Syndrome (i.e.: paraneoplastic hemopathies, hypercalcemia, coagulopathies, fatigue, weakness, cachexia, chronic nausea, anorexia, and early satiety among others) could be ameliorated ${ }^{(86)}$. Fish oil EPA-enriched supplement $(1.09 \mathrm{~g} / \mathrm{d})$ may reverse cachexia in advanced pancreatic adenocarcinoma, and showed weight-gain at both 3 $(1 \mathrm{~kg})$ and 7 weeks $(2 \mathrm{~kg})^{(87)}$. Increased intakes of dietary ALA may increase the risk of advanced prostate cancer, whereas EPA and DHA intakes may reduce the risk of total and advanced prostate cancer ${ }^{(88)}$.

Until now, we have listed a number of studies that have clearly remarked the benefits of fish oil $n$-3 LC-PUFAs. However, some concerns about potential health risks derived from the environmental pollutants and contaminants found in fish have been also raised. One of the most dangerous contaminants is methylmercury ( $\mathrm{MeHg}$ ). Mercury is emitted into the atmosphere from several sources. From the atmosphere, mercury cycles from rainwater into lakes and oceans, where it is converted by the action of microorganisms into organic $\mathrm{MeHg}$, which is well absorbed and actively transported into tissues by a widely distributed carrier protein ${ }^{(89,90)}$. The concentration of $\mathrm{MeHg}$ in any given fish species depends on the degree of local environmental contamination and on the predatory nature and lifespan of the species. The concentration of $\mathrm{MeHg}$ in fish is increased by fish eating other fish for food. Fish that are not predatory, shorter-lived or smaller species, such as sardines, salmon, flounder, canned light tuna and shrimp, therefore have very low levels of $\mathrm{MeHg}$. By contrast, longer-living and predatory fish such as shark, tuna, swordfish and orange roughly have higher levels of $\mathrm{MeHg}$. Interestingly, the much-maligned farmed fish have the lowest levels of MeHg. Although MeHg per se is very neurotoxic, in fish $\mathrm{MeHg}$ is bound to cysteine, and this compound has a tenth of the toxicity of pure $\mathrm{MeHg}^{(91,92)}$. $\mathrm{MeHg}$ can bind to the sulfhydryl groups of enzymes, ion channels, and receptors, inhibiting important antioxidant systems and increasing the production of reactive oxygen species and free radicals ${ }^{(90,93)}$. Health effects of very high doses of MeHg exposure are well-documented and include paresthesias, ataxia, and sensory abnormalities in adults, and delayed cognitive and neuromuscular development in children following in utero exposure $^{(90,94)}$. MeHg crosses the placenta, and exposure to the fetus is a function of maternal exposure ${ }^{(95)}$. Following very high gestational exposure, severe neurodevelopmental abnormalities can occur in children. However, the health effects of chronic low level mercury exposure are scarcely well-established.

Estimated $n$-3 LC-PUFAs benefits outweighed cardiovascular and neurodevelopmental $\mathrm{MeHg}$ risks for some species (farmed salmon, herring, trout); however, the opposite was true for others (swordfish, shark). Other species were associated with a small net benefit (flounder, canned light tuna) or a small net risk (canned white tuna, halibut) ${ }^{(96)}$.

More typical MeHg exposures from fish consumption are far lower. Among US women of childbearing age, the median 
levels of hair mercury were $0.19 \mathrm{ppm}$ overall, and $0.34 \mathrm{ppm}$ among women who consumed more than three servings of fish per month ${ }^{(97)}$. These low exposure levels do not produce clinically detectable neurologic symptoms or signs in children. In studies in the Faroe Islands ${ }^{(98,99)}$, New Zealand ${ }^{(100,101)}$, and Poland ${ }^{(102)}$, higher gestational mercury exposure was associated with lower scores on some neurologic tests, but not on most of them. In the Seychelles, however, higher gestational $\mathrm{MeHg}$ exposure was associated with higher scores on some neurologic tests ${ }^{(103,104)}$. Maternal fish intake during gestation was associated with better visual recognition memory scores, while maternal hair mercury was associated with lower visual recognition memory scores ${ }^{(105)}$, suggesting that overall fish consumption (which provides DHA, likely beneficial for neurodevelopment) and $\mathrm{MeHg}$ exposure may have opposing effects. Gestational mercury exposure was not associated with neurodevelopmental scores, but it was associated with better neurodevelopmental scores in other human populations $^{(106)}$.

It should be useful in establishing advisories for a wide variety of commercially available and locally caught fish, assuming that the requisite $\mathrm{MeHg}$ and $n-3$ LC-PUFAs data are available ${ }^{(95,107-112)}$. This caution should be extended to other foods fortified with fish oil $n$-3 LC-PUFAs, such as eggs and milk. However, exceeding the tolerable daily intake was just noticed for heavy seafood consumers. Wild and farmed fish are generally both similar in $n$-3 LC-PUFAs contents but may vary in terms of potential toxins, but they affected proteins and not fatty acids.

Accordingly, the Environmental Protection Agency published a focused advisory for women of childbearing age, nursing mothers, and young children ${ }^{(113)}$. The allowable upper limit of daily intake, for methylmercury of $0 \cdot 1 \mu \mathrm{g} / \mathrm{kg}$ per d (approx. $50 \mu \mathrm{g} /$ week for a $70 \mathrm{~kg}$ woman) ${ }^{(95)}$. Four fish species (shark, swordfish, king mackerel, and tilefish) exceed this limit in a single serving. So, women of childbearing age, nursing mothers, and young children should avoid these specific species, but they could consume a variety of other fish up to 2 servings/week (including up to 1 serving/ week of albacore tuna) to receive the important health benefits ${ }^{(112)}$. The US Institute of Medicine recommended that pregnant women restrict their intake of fish with a higher MeHg content (shark, tuna, or swordfish) to 1 meal per 2 weeks; however, these women can eat 2-3 meals of other fish per week (sardines, salmon, or shrimp) ${ }^{(91)}$. The importance of this conservative reference dose for health effects in adults remains still unclear ${ }^{(113)}$

The results of studies of mercury exposure and cardiovascular disease incidence in adults provide inconclusive evidence for cardiovascular toxicity of mercury exposure. Of note, in the only two studies that observed positive associations between mercury exposure and cardiovascular risk, the net effect of fish consumption was still beneficial ${ }^{(114-116)}$

Sensorimotor symptoms in adults, most commonly paresthesias, can be seen following very high methylmercury exposure from accidents ${ }^{(90,94,117)}$ or prolonged high intakes of mercury-containing fish (1-2 fish servings per day, including species high in mercury, for $>10$ years $)^{(54)}$. Such symptoms are typically reversible when mercury exposure is reduced. Evidence suggests that fish consumption may favorably affect clinical neurologic outcomes in adults, including ischemic stroke ${ }^{(118)}$, cognitive decline and dementia ${ }^{(119)}$, and depression and other neuropsychiatric disorders ${ }^{(120,121)}$.

Other potential contaminants in fish such as dioxins and polychlorinated biphenyls could potentially increase the risk of cancer. An analysis of the potential harmful effects of these contaminants in fish versus the benefits of omega-3 fatty acids has, however, concluded that the levels of dioxins and polychlorinated biphenyls in fish are low, and potential carcinogenic and other effects are outweighed by potential benefits of fish intake ${ }^{(89,122)}$.

To sum up, the balance of benefit vs. risk is most favourable for oily fish species which contain higher amounts of $n-3$ LC-PUFAs, compared with lean fish, which are generally lower in $n$-3 LC-PUFAs.

\section{Plant omega-3 fatty acids}

To achieve recommended alpha-linoleic acid (ALA) intakes, food sources including flaxseed and flaxseed oil, walnuts and walnut oil, and canola oil are recommended. Short-term trials $(6-12 \mathrm{wk})$ in healthy participants mostly showed no or inconsistent effects of ALA intake $(1.2-3.6 \mathrm{~g} / \mathrm{d})$ on blood lipids, LDL oxidation, lipoprotein A, and apolipoproteins A-I and $\mathrm{B}$. There was a protective effect against nonfatal myocardial infarction ${ }^{(123-128)}$. However, no protective associations were observed between ALA status and risk of heart failure, atrial fibrillation, and sudden death ${ }^{(129-134)}$. Dietary ALA and $\mathrm{EPA}+$ DHA had different physiologic effects on fasting TAG concentrations, and susceptibility of LDL to oxidation ${ }^{(135)}$. Findings from long-term trials of ALA supplementation were awaited to answer the question whether food-based or higher doses of ALA could be important for cardiovascular health in cardiac patients and the general population. ALA derived from plant sources decreased the risk for mild dementia among elderly people ${ }^{(136)}$. Plant sources of dietary $n$-3 LC-PUFAs may have a protective effect on bone metabolism via a decrease in bone resorption in the presence of consistent levels of bone formation ${ }^{(137)}$.

Flaxseed is a rich source of ALA (35\% of its mass as oil, of which $55 \%$ is ALA), fibre and lignans, making it a potentially attractive functional food for modulating cardiovascular risk. Flaxseed oil intake increases ALA and EPA plasma levels, but not DHA, did not affect glycaemia ${ }^{(138)}$, had an hypotensive effect $^{(139)}$, a modest but short lived LDL-cholesterol lowering effect, yet reduced lipoprotein A, improved insulin sensitivity in hyperlipidemic adults ${ }^{(127)}$, had no effect on plasma adiponectin concentration in dyslipidemic men ${ }^{(140)}$, did not affect serum lipids, except for a slight reduction in serum TAG, did not decrease CVD risk by altering lipoprotein particle size or plasma concentrations, and did not compromise antioxidant status $^{(141,142)}$. Flaxseed oil did not have antioxidant activity except they suppressed oxygen radical production by white blood cells. An intake of $\leq 9.5 \mathrm{~g} / \mathrm{d}$ flaxseed oil ALA did not alter the functional activity of neutrophils, monocytes, or lymphocytes, but it changed the fatty acid composition of 
mononuclear cells. Flaxseed oil ALA doses $\leq 14 \mathrm{~g} / \mathrm{d}$ did not affect inflammatory mediators/markers, but $\geq 14 \mathrm{~g} / \mathrm{d}$ reduced inflammatory mediators/markers and platelet aggregation, and increased platelet activating inhibitor-1 and bleeding time ${ }^{(143)}$. Therefore, flaxseed and its components improve cardiovascular health. Fibre contents of flaxseed increased bowel movements per week ${ }^{(142)}$, and suppression of atherosclerosis wa just due to its lignan content ${ }^{(143)}$.

Feeding healthy term infants' soy-based formula DHA and ARA supplemented at concentrations similar to human milk significantly increased circulating levels of DHA and ARA in total red blood cells and plasma phospholipids. Supplementation did not affect the tolerance of formula or the incidence of adverse events ${ }^{(144)}$.

Dietary intake of rapeseed ALA, EPA or DHA for 3 weeks led to a significant enrichment these fatty acids in the LDL particles, with dietary EPA preferentially incorporated. ALA enrichment did not enhance LDL oxidizability, whereas the effects of EPA and DHA on LDL oxidation were inconsistent, possibly in part due to further changes in LDL fatty acid composition $^{(145)}$.

\section{Omega-3 fatty acids enriched dairy products}

The consumption of $500 \mathrm{~mL} / \mathrm{d}$ for $6 \mathrm{wk}$ of an enriched semiskimmed milk ( $400 \mathrm{mg}$ of EPA and DHA) decreased TAG and increased HDL-cholesterol serum levels ${ }^{(146)}$. An 8-wk supplementation of $500 \mathrm{~mL} / \mathrm{d}$ enriched semi-skimmed dairy products $(60 \mathrm{mg} / 100 \mathrm{~mL} \mathrm{EPA}$ and DHA $)$ decreased LDLcholesterol and TC serum levels ${ }^{(147,148)}$. The consumption of $3 \mathrm{~g} / \mathrm{d} n-3$ LC-PUFAs-supplemented dairy products for fifteen weeks decreased cardiovascular risk factors (TC, TAG, high HDL-cholesterol, low LDL/HDL ratio) ${ }^{(149)}$. The consumption of $n$-3 LC-PUFAs milkshake providing $2.0 \mathrm{~g}$ EPA and $2.7 \mathrm{~g}$ DHA (ratio 2:3) had an attenuating effect on augmentation index and stiffness index ${ }^{(150)}$. Seven-month consumption of $500 \mathrm{~mL} / \mathrm{d}$ of a PUFA enriched dairy drink (60\% olive oil, $20 \%$ peanut, and $20 \%$ sunflower), containing a quarter of the saturated fat present in standard whole milk, decreased serum levels of total cholesterol and LDL-cholesterol, without reducing caloric intake, in 3-9 year-old children ${ }^{(151)}$. These effects were not observed after administration of EPA and DHA capsules ${ }^{(152)}$, showing that the vehicle of administration (milk) also plays a role in the produced effects.

The consumption of a PUFA enriched dairy $500 \mathrm{~mL} / \mathrm{d}$ of the test milk for 1 year in $29725-65 \mathrm{y}$-o subjects with moderate $\mathrm{CV}$ risk increased serum HDL-cholesterol levels, and decreased TG, TC, and LDL-cholesterol ${ }^{(153)}$. When this intervention was carried out in patients with peripheral vascular disease, TC apolipoprotein B levels decreased, mainly in patients with high cholesterol values, but also increased the walking distance before the onset of pain, a method to measure the intensity of this illness ${ }^{(154)}$. Similar results were obtained in patients with history of myocardial infarction ${ }^{(155)}$.

Finally, 3-month consumption of $186 \mathrm{mg} / \mathrm{d}$ EPA and DHA in skimmed milk reduced TC, LDL-cholesterol, and TAG serum levels ${ }^{(156)}$. The average inclusion of $300 \mathrm{mg}$ of EPA and DHA in the milk produced $25-50 \%$ enrichment in the plasma levels of the fatty acids after a minimum period of 6 weeks, because milk is a very efficient carrier for fat absorption, enhancing the bioavailability of $n-3$ LC-PUFAs $^{(7,142,143,156)}$. The intake of ALA, EPA or DHA-supplemented margarine led to a significant enrichment of the LDL with the respective $n$-3 LC-PUFAs. ALA, EPA, or DHA intake did not affect fasting serum concentrations of total and LDL-cholesterol, but fasting serum TAG concentrations significantly decreased. DHA intake significantly increased serum HDL cholesterol, whereas no changes were found with ALA or EPA intake (124) $^{\text {These }}$

These intervention studies in patients show that the inclusion of $n$-3 LC-PUFAs enriched dairy products in the usual dietary pattern increases the ability to control the CVD risk factors, and also improve clinical outcomes.

\section{Animal-derived food omega-3 fatty acids}

Poultry meat contributes small but worthwhile amounts of EPA and DHA. Studies on EPA and DHA contents of animalderived foods mainly use fish oil to enrich these diets. This enrichment has the potential to provide a daily intake of EPA and DHA of about $230 \mathrm{mg}$ to the Western adult diet, with poultry meat providing the largest amount $(74 \mathrm{mg})^{(157)}$ A significant increase in $n$-3 LC-PUFAs levels in beef from cattle fed rations supplemented with flaxseed has been demonstrated $^{(158)}$.

Available literature indicates that the levels of EPA and DHA in food products may be increased more, if the animals' diet was supplemented with fish products rather than seed products. Sometimes, organoleptic properties of food products may be compromised. It has been suggested that omega-3 fatty acids may be enriched in pork by feeding swine with tuna oil, but sensory properties and shelf life decreased $^{(159,160)}$. However, adverse effects could not appear, i.e. addition of fish oils to Bruehwurst sausages increased the $n$-3 LC-PUFAs contents without changes on sensory properties, and just showed off-flavours, not always described as 'fishy' (161)

A standard egg contains a ratio of $n-3$ LC-PUFAs to total fat less than $1 \%$. By feeding laying hens with grains, soybean and ?axseed rich in ALA, $n$-3 LC-PUFAs content per egg can be increased to 6 times than the standard eggs. Three $n$ - 3 LC-PUFAs-enriched eggs provided approximately the same amount of $n-3$ PUFA as one meal with fish ${ }^{(162)}$. Consumption of $n$-3 LC-PUFAs-enriched eggs reduced systolic blood pressure, but had no effect on BMI, WHR, waist circumference and diastolic blood pressure, with no change in the daily intake of energy, protein, carbohydrate, total fat, SFA and MUFA, but increased PUFA and TC blood levels, and decreased plasma fasting insulin and CRP levels. Reasonable consumption of $n$-3 LC-PUFAs enriched eggs (hen feed supplemented at $5 \%$ tuna oil, and enriched eggs contained nine times more $n$-3 PUFA than usual eggs, mainly DHA) was associated with a significant decrease in 16-18\% decrease in serum triglycerides, but with no significant difference in serum LDL- and HDL-cholesterol. These eggs could be a palatably acceptable source of $n$-3 LC-PUFAs ${ }^{(163)}$. Feeding hens with 
microalgae-rich diet, an improvement in DHA contents was obtained, avoiding unpleasant flavours associated with fish oil supplementation ${ }^{(164)}$.

It is interesting, however, to know the impact of the chow formulation used on farms and breeding centres on the nutritional value of the animal products, and their effect on the health of consumers. The consequences of modifications in the composition of animal foods on the value of derived products consumed by humans are more marked when single-stomach animals are concerned than multi-stomach animals, because hydrogenating intestinal bacteria of the latter group transform a large proportion of PUFA in their food into SFA, among others, thus depriving them of any biological interest ${ }^{(165)}$.

\section{Krill oil omega-3 fatty acids}

Antarctic krill, Euphausia superba, is a marine crustacean that has not been a traditional food in the human diet. Krill is a rich source of high-quality protein, with the advantage over other animal proteins of being low in fat and a rich source of EPA and DHA. Antioxidant levels in krill are higher than in fish, suggesting benefits against oxidative damage. Finally, the waste generated by the processing of krill into edible products can be developed into valueadded products ${ }^{(166)}$.

Plasma EPA and DHA concentrations increased significantly, and blood urea decreased after overweight and obese men and women received capsules containing $2 \mathrm{~g} / \mathrm{d}$ of krill oil for 4 weeks. Nor other changes, neither adverse effects were detected $^{(167)}$. Patients treated 3 mo with $1 \mathrm{~g} / \mathrm{d}$ and $1.5 \mathrm{~g} / \mathrm{d}$ krill oil demonstrated that krill oil is effective for the management of hyperlipidemia by significantly reducing total cholesterol, LDL, and triglycerides, and increasing HDL levels. At lower and equal doses, krill oil was significantly more effective than fish oil for the reduction of glucose, triglycerides, and LDL levels ${ }^{(168)}$. Neptune Krill Oil may significantly reduced dysmenorrhea and the emotional symptoms of premenstrual syndrome and showed to be significantly more effective than omega-3 fish oil ${ }^{(169)}$.

\section{Seal oil omega-3 fatty acids}

Seal oil supplementation in healthy, normocholesterolemic subjects decreased the $n-6 / n-3$ ratio and increased EPA, DHA, and DPA and the ratio of EPA/AA and DHA/AA in the serum, while exhibited a modest beneficial effect on fibrinogen and CRP levels ${ }^{(170)}$. No change was observed in body weight, fasting blood glucose, renal function and blood cells of patients with nonalcoholic fatty liver disease associated with hyperlipidemia after an intervention with $2 \mathrm{~g} n$-3 LCPUFAs from seal oils, three times a day, $24 \mathrm{wk}$. Liver alanine aminotransferase and TAG blood levels decreased after the intervention. Fatty liver regression was observed in $19.7 \%$ of the patients, and an overall reduction was found in $53.0 \%$. No serious adverse events occurred in all the patients who completed the treatment ${ }^{(171)}$.

\section{Discussion}

In this review, findings were classified according to the dietary source of the omega- 3 fatty acids, and their benefits and the risks for the public health.

Algal omega-3 fatty acids are DHA and DPA, and their main effects are a decrease of TAG and VLDL and a slightly increase of HDL and LDL-cholesterol plasma levels, as well as Factor VII coagulant activity. Up to date, no adverse effects have been observed.

Fish oils are the most common source of source of omega-3 fatty acids, mainly EPA and DHA. It has been pointed out protective and beneficial effects of these fatty acids on hearth health, CVD, blood lipid profile, T2DM, inflammatory and renal diseases, maternal and child health, CNS function, elderly, psychiatric disorders, several cancers, and other illnesses. Several studies suggested an increased risk of T2DM with the intake of marine $n-3$ LC-PUFAs, especially with higher intakes. Another potential health risk derived from the environmental contaminants found in fish.

Plant omega-3 fatty acids are the main source of ALA, which increases blood DHA and ARA levels, improves insulin sensitivity, has a very small hypotensive effect, and a protective effect on bone metabolism. Other benefits are still inconsistent. The main question is whether dietary intake of ALA can provide enough EPA and DHA amounts.

Enriched dairy products are a good vehicle to provide omega-3 fatty acids. The benefits are addressed to improve the blood lipid profile, arterial stiffness, inflammation, and oxidative stress markers, and to decrease CVD risks. No adverse effects have been yet described.

Animal-derived food omega-3 fatty acids contribute to EPA and DHA levels. Enriched eggs are one of the most common sources of animal-derived food omega-3 fatty acids. The benefits and risks on the public health depend on the chow formulation used in farms, and the type of fats fed by the animals. The only adverse effects may be decreased meat sensory properties and shelf life.

Krill is a rich source of high-quality protein, also low in fat and a rich source of EPA and DHA. The benefits are effects against oxidative damage, increase of HDL, EPA and DHA blood levels, decrease of LDL, TAG, and urea levels, as well as dysmenorrhea and premenstrual symptoms, and the waste generated by its processing into edible products can be developed into value-added products. No adverse effects have been described.

Seal oil contributes to increase EPA, DHA, DPA, and TAG blood levels. No adverse effects have been described. and disclosures

\section{Acknowledgements and disclosures}

The preparatory meetings for this series of reviews on fat and health were funded by Puleva Food. Neither Josep A. Tur nor Maria del Mar Bibiloni, Antoni Sureda or Antoni Pons have conflicts of interest to disclose. Josep A. Tur and Maria del Mar Bibiloni contributed to the design of the strategy for the literature search, double screened and selected the retrieved 
documents. Authors acknowledge Angel Gil from the University of Granada the support provided to select and retrieve several documents. Antoni Sureda and Antoni Pons provided previous literature searches and analysis. Josep A. Tur prepared the main outline of the manuscript and all authors contributed to the preparation of the manuscript.

\section{References}

1. EFSA (2009) Opinion of the scientific panel on dietetic products, nutrition and allergies on a request from the Commission related to labeling reference intake values for $n-3$ and n-6 polyunsaturated fatty acids. EFSA J 1176, 1-11.

2. Kris-Etherton PM, Harris WS, Appel LJ, et al. (2002) Nutrition Committee, Fish consumption, fish oil, omega-3 fatty acids, and cardiovascular disease. Circulation 106, 2747-2757.

3. Kris-Etherton PM, Harris WS, Appel LJ, et al. (2003) Omega3 fatty acids and cardiovascular disease: new recommendations from the American Heart Association. Arterioscler Thromb Vasc Biol 23, 1, 51-152.

4. The World Health Organisation (2003) Diet nutrition and the prevention of chronic diseases. Report of the WHO/ FAO Joint Expert Consultation, WHO, Technical Report Series 916

5. Pawlosky RJ, Hibbeln JR, Novotny JA, et al. (2001) Physiological compartmental analysis of alpha-linolenic acid metabolism in adult humans. J Lipid Res 42, 1257-1265.

6. Arterburn LM, Hall EB \& Oken H (2006) Distribution, interconversion, and dose response of n-3 fatty acids in humans. Am J Clin Nutr 83, 1467S-1476S.

7. Lopez-Huertas E (2010) Health effects of oleic acid and long chain omega-3 fatty acids (EPA and DHA) enriched milks. A review of intervention studies. Pharmacol Res $\mathbf{6 1}$, 200-207.

8. Neff LM, Culiner J, Cunningham-Rundles S, et al. (2011) Algal docosahexaenoic acid affects plasma lipoprotein particle size distribution in overweight and obese adults. J Nutr 141, 207-213.

9. Geppert J, Kraft V, Demmelmair H, et al. (2006) Microalgal docosahexaenoic acid decreases plasma triacylglycerol in normolipidaemic vegetarians: a randomised trial. $\mathrm{Br} \mathrm{J} \mathrm{Nutr}$ 95, 779-786.

10. Sanders TA, Gleason K, Griffin B, et al. (2006) Influence of an algal triacylglycerol containing docosahexaenoic acid (22: 6n-3) and docosapentaenoic acid (22:5n-6) on cardiovascular risk factors in healthy men and women. BrJ Nutr 95, 525-531.

11. Harris WS, Pottala JV, Sands SA, et al. (2007) Comparison of the effects of fish and fish-oil capsules on the $\mathrm{n} 3$ fatty acid content of blood cells and plasma phospholipids. Am J Clin Nutr 86, 1621-1625.

12. Milte CM, Coates AM, Buckley JD, et al. (2008) Dosedependent effects of docosahexaenoic acid-rich fish oil on erythrocyte docosahexaenoic acid and blood lipid levels. Br J Nutr 99, 1083-1088.

13. Peoples GE, McLennan PL, Howe PR, et al. (2008) Fish oil reduces heart rate and oxygen consumption during exercise. J Cardiovasc Pharmacol 52, 540-547.

14. Harrison RA, Sagara M, Rajpura A, et al. (2004) Can foods with added soya-protein or fish-oil reduce risk factors for coronary disease? A factorial randomised controlled trial. Nutr Metab Cardiovasc Dis 14, 344-350.
15. Singer P, Melzer S, Goschel M, et al. (1990) Fish oil amplifies the effect of propranolol in mild essential hypertension. Hypertension 16, 682-691.

16. Din JN, Harding SA, Valerio CJ, et al. (2008) Dietary intervention with oil rich fish reduces platelet-monocyte aggregation in man. Atherosclerosis 197, 290-296.

17. Pirich C, Gaszo A, Granegger S, et al. (1999) Effects of fish oil supplementation on platelet survival and ex vivo platelet function in hypercholesterolemic patients. Thromb Res 96, $219-227$.

18. Metcalf RG, James MJ, Gibson RA, et al. (2007) Effects of fish-oil supplementation on myocardial fatty acids in humans. Am J Clin Nutr 85, 1222-1228.

19. Mehra MR, Lavie CJ, Ventura HO, et al. (2006) Fish oils produce anti-inflammatory effects and improve body weight in severe heart failure. J Heart Lung Transplant 25, 834-838.

20. Kaul U, Sanghvi S, Bahl VK, et al. (1992) Fish oil supplements for prevention of restenosis after coronary angioplasty. Int J Cardiol 35, 87-93.

21. Erkkilä AT, Lichtenstein AH, Mozaffarian D, et al. (2004) Fish intake is associated with a reduced progression of coronary artery atherosclerosis in postmenopausal women with coronary artery disease. Am J Clin Nutr 80, 626-632.

22. Seierstad SL, Seljeflot I, Johansen O, et al. (2005) Dietary intake of differently fed salmon; the influence on markers of human atherosclerosis. Eur J Clin Invest 35, 52-59.

23. Ueshima H, Stamler J, Elliott P, et al. (2007) Food omega-3 fatty acid intake of individuals (total, linolenic acid, long-chain) and their blood pressure: INTERMAP study. Hypertension 50, 313-319.

24. Berry JD, Prineas RJ, van Horn L, et al. (2010) Dietary fish intake and incident atrial fibrillation (from the Women's Health Initiative). Am J Cardiol 105, 844-848.

25. Brouwer IA, Zock PL, Camm AJ, et al. (2006) Effect of fish oil on ventricular tachyarrhythmia and death in patients with implantable cardioverter defibrillators: the Study on Omega-3 Fatty Acids and Ventricular Arrhythmia (SOFA) randomized trial. JAMA 295, 2613-2619.

26. Fahs CA, Yan H, Ranadive S, et al. (2010) The effect of acute fish-oil supplementation on endothelial function and arterial stiffness following a high-fat meal. Appl Physiol Nutr Metab 35, 294-302.

27. Poppitt SD, Howe CA, Lithander FE, et al. (2009) Effects of moderate-dose omega-3 fish oil on cardiovascular risk factors and mood after ischemic stroke: a randomized, controlled trial. Stroke 40, 3485-3492.

28. DeGiorgio CM, Miller P, Meymandi S, et al. (2008) n-3 fatty acids (fish oil) for epilepsy, cardiac risk factors, and risk of SUDEP: clues from a pilot, double-blind, exploratory study. Epilepsy Behav 13, 681-684.

29. Watanabe N, Watanabe Y, Kumagai M, et al. (2009) Administration of dietary fish oil capsules in healthy middle-aged Japanese men with a high level of fish consumption. Int $J$ Food Sci Nutr 60, Suppl 5, 136-142.

30. Piolot A, Blache D, Boulet L, et al. (2003) Effect of fish oil on LDL oxidation and plasma homocysteine concentrations in health. J Lab Clin Med 141, 41-49.

31. Mostad IL, Bjerve KS, Lydersen S, et al. (2008) Effects of marine $\mathrm{n}-3$ fatty acid supplementation on lipoprotein subclasses measured by nuclear magnetic resonance in subjects with type II diabetes. Eur J Clin Nutr 62, 419-429.

32. Khandelwal S, Demonty I, Jeemon P, et al. (2009) Independent and interactive effects of plant sterols and fish oil n-3 long-chain polyunsaturated fatty acids on the plasma lipid 
profile of mildly hyperlipidaemic Indian adults. $\mathrm{Br} J \mathrm{Nutr}$ 102, 722-732.

33. Feskens EJ, Virtanen SM, Rasanen L, et al. (1995) Dietary factors determining diabetes and impaired glucose tolerance. A 20-year follow-up of the Finnish and Dutch cohorts of the Seven Countries Study. Diabetes Care 18, 1104-1112.

34. Patel PS, Sharp SJ, Luben RN, et al. (2009) Association between type of dietary fish and seafood intake and the risk of incident type 2 diabetes: the European prospective investigation of cancer (EPIC)-Norfolk cohort study. Diabetes Care 32, 1857-1863.

35. Feskens EJ, Bowles CH \& Kromhout D (1991) Inverse association between fish intake and risk of glucose intolerance in normoglycemic elderly men and women. Diabetes Care 14, 935-941.

36. Kaushik M, Mozaffarian D, Spiegelman D, et al. (2009) Long chain omega-3 fatty acids, fish intake, and the risk of type 2 diabetes mellitus. Am J Clin Nutr 90, 613-620.

37. Friday KE, Childs MT, Tsunehara CH, et al. (1989) Elevated plasma glucose and lowered triglyceride levels from omega-3 fatty acid supplementation in type II diabetes. Diabetes Care 12, 276-281.

38. Rivellese AA, Maffettone A, Iovine C, et al. (1996) Longterm effects of fish oil on insulin resistance and plasma lipoproteins in NIDDM patients with hypertriglyceridemia. Diabetes Care 19, 1207-1213.

39. Luo J, Rizkalla SW, Vidal H, et al. (1998) Moderate intake of n-3 fatty acids for 2 months has no detrimental effect on glucose metabolism and could ameliorate the lipid profile in type 2 diabetic men. Results of a controlled study. Diabetes Care 21, 717-724.

40. Borkman M, Chisholm DJ, Furler SM, et al. (1989) Effects of fish oil supplementation on glucose and lipid metabolism in NIDDM. Diabetes 38, 1314-1319.

41. Djoussé L, Gaziano JM, Buring JE, et al. (2011) Dietary omega-3 fatty acids and fish consumption and risk of type 2 diabetes. Am J Clin Nutr 93, 143-150.

42. Belalcazar LM, Reboussin DM, Haffner SM, et al. (2010) Marine omega-3 fatty acid intake: associations with cardiometabolic risk and response to weight loss intervention in the Look AHEAD Study. Diabetes Care 33, 197-199.

43. Giacco R, Cuomo V, Vessby B, et al. (2007) Fish oil, insulin sensitivity, insulin secretion and glucose tolerance in healthy people: is there any effect of fish oil supplementation in relation to the type of background diet and habitual dietary intake of n-6 and n-3 fatty acids? Nutr Metab Cardiovasc Dis 17, 572-580.

44. Mostad IL, Bjerve KS, Basu S, et al. (2009) Addition of n-3 fatty acids to a 4-hour lipid infusion does not affect insulin sensitivity, insulin secretion, or markers of oxidative stress in subjects with type 2 diabetes mellitus. Metabolism 58, $1753-1761$.

45. Navas-Carretero S, Pérez-Granados AM, Schoppen S, et al. (2009) An oily fish diet increases insulin sensitivity compared to a red meat diet in young iron-deficient women. Br J Nutr 102, 546-553.

46. Kew S, Mesa MD, Tricon S, et al. (2004) Effects of oils rich in eicosapentaenoic and docosahexaenoic acids on immune cell composition and function in healthy humans. $A m \mathrm{~J}$ Clin Nutr 79, 674-681.

47. Ramel A, Martinez JA, Kiely M, et al. (2010) Effects of weight loss and seafood consumption on inflammation parameters in young, overweight and obese European men and women during 8 weeks of energy restriction. Eur J Clin Nutr 64, 987-993.
48. Ramel A, Martinez JA, Kiely M, et al. (2010) Moderate consumption of fatty fish reduces diastolic blood pressure in overweight and obese European young adults during energy restriction. Nutrition 26, 168-174.

49. Zhang J, Wang C, Li L, et al. (2010) Inclusion of Atlantic salmon in the Chinese diet reduces cardiovascular disease risk markers in dyslipidemic adult men. Nutr Res 30, 447-454.

50. Mickleborough TD, Murray RL, Ionescu AA, et al. (2003) Fish oil supplementation reduces severity of exerciseinduced bronchoconstriction in elite athletes. Am J Respir Crit Care Med 168, 1181-1189.

51. Bowden RG, Wilson RL, Deike E, et al. (2009) Fish oil supplementation lowers C-reactive protein levels independent of triglyceride reduction in patients with end-stage renal disease. Nutr Clin Prac 24, 508-512.

52. Lankinen M, Schwab U, Erkkilä A, et al. (2009) Fatty fish intake decreases lipids related to inflammation and insulin signalling: a lipidomics approach. PLoS One 4, e5258.

53. Lopez-Garcia E, Schulze MB, Manson JE, et al. (2004) Consumption of (n-3) fatty acids is related to plasma biomarkers of inflammation and endothelial activation in women. J Nutr 134, 1806-1811.

54. Xiong J, Zhu S, Zhou Y, et al. (2009) Regulation of omega-3 fish oil emulsion on the SIRS during the initial stage of severe acute pancreatitis. J Huazhong Univ Sci Technolog Med Sci 29, 35-38.

55. Lau CS, Morley KD \& Belch JJ (1993) Effects of fish oil supplementation on non-steroidal anti-inflammatory drug requirement in patients with mild rheumatoid arthritis - a double-blind placebo controlled study. Br J Rheumatol 32, 982-989.

56. Maroon JC \& Bost JW (2006) Omega-3 fatty acids (fish oil) as an anti-inflammatory: an alternative to nonsteroidal antiinflammatory drugs for discogenic pain. Surg Neurol 65 , 326-331.

57. Galarraga B, Ho M, Youssef HM, et al. (2008) Cod liver oil (n-3 fatty acids) as an non-steroidal anti-inflammatory drug sparing agent in rheumatoid arthritis. Rheumatology (Oxford) 47, 665-669.

58. Caughey GE, James MJ, Proudman SM, et al. (2010) Fish oil supplementation increases the cyclooxygenase inhibitory activity of paracetamol in rheumatoid arthritis patients. Complement Ther Med 18, 171-174.

59. Dry J \& Vincent D (1991) Effect of a fish oil diet on asthma: results of a 1-year double-blind study. Int Arch Allergy Appl Immunol 95, 156-157.

60. Pot GK, Brouwer IA, Enneman A, et al. (2009) No effect of fish oil supplementation on serum inflammatory markers and their interrelationships: a randomized controlled trial in healthy, middle-aged individuals. Eur J Clin Nutr 63, 1353-1359.

61. Michaeli B, Berger MM, Revelly JP, et al. (2007) Effects of fish oil on the neuro-endocrine responses to an endotoxin challenge in healthy volunteers. Clin Nutr 26, 70-77.

62. Bouwens M, van de Rest O, Dellschaft N, et al. (2009) Fish-oil supplementation induces antiinflammatory gene expression profiles in human blood mononuclear cells. Am J Clin Nutr 90, 415-424.

63. Moghadamnia AA, Mirhosseini N, Abadi MH, et al. (2010) Effect of Clupeonella grimmi (anchovy/kilka) fish oil on dysmenorrhoea. East Mediterr Health J 16, 408-413.

64. Franke C, Demmelmair H, Decsi T, et al. (2010) Influence of fish oil or folate supplementation on the time course of plasma redox markers during pregnancy. Br J Nutr 103, 1648-1656. 
65. Dunstan JA, Simmer K, Dixon G, et al. (2008) Cognitive assessment of children at age 2(1/2) years after maternal fish oil supplementation in pregnancy: a randomized controlled trial. Arch Dis Child Fetal Neonatal Ed 93, F45-F50.

66. Schnappinger M, Sausenthaler S, Linseisen J, et al. (2009) Fish consumption, allergic sensitization and allergic diseases in adults. Ann Nutr Metab 54, 67-74.

67. Prescott SL, Barden AE, Mori TA, et al. (2007) Maternal fish oil supplementation in pregnancy modifies neonatal leukotriene production by cord-blood-derived neutrophils. Clin Sci (Lond) 113, 409-416.

68. Smithers LG, Markrides M \& Gibson RA (2010) Human milk fatty acids from lactating mothers of preterm infants: a study revealing wide intra- and inter-individual variation. Prostaglandins Leukot Essent Fatty Acids 83, 9-13.

69. Furuhjelm C, Warstedt K, Larsson J, et al. (2009) Fish oil supplementation in pregnancy and lactation may decrease the risk of infant allergy. Acta Paediatr 98, 1461-1467.

70. Lauritzen L, Christensen JH, Damsgaard CT, et al. (2008) The effect of fish oil supplementation on heart rate in healthy Danish infants. Pediatr Res 64, 610-614.

71. Fortier M, Tremblay-Mercier J, Plourde M, et al. (2010) Higher plasma n-3 fatty acid status in the moderately healthy elderly in southern Québec: higher fish intake or aging-related change in n-3 fatty acid metabolism? Prostaglandins Leukot Essent Fatty Acids 82, 277-280.

72. van de Rest O, Geleijnse JM, Kok FJ, et al. (2008) Effect of fish oil on cognitive performance in older subjects: a randomized, controlled trial. Neurology 71, 430-438.

73. van de Rest O, Geleijnse JM, Kok FJ, et al. (2009) Effect of fish oil supplementation on quality of life in a general population of older Dutch subjects: a randomized, double-blind, placebo-controlled trial. J Am Geriatr Soc 57, 1481-1486.

74. Astorg P, Couthouis A, Bertrais S, et al. (2008) Association of fish and long-chain n-3 polyunsaturated fatty acid intakes with the occurrence of depressive episodes in middleaged French men and women. Prostaglandins Leukot Essent Fatty Acids 78, 171-182.

75. Ramel A, Parra D, Martinez JA, et al. (2009) Effects of seafood consumption and weight loss on fasting leptin and ghrelin concentrations in overweight and obese European young adults. Eur J Nutr 48, 107-114.

76. van de Rest O, Geleijnse JM, Kok FJ, et al. (2008) Effect of fish-oil supplementation on mental well-being in older subjects: a randomized, double-blind, placebo-controlled trial. Am J Clin Nutr 88, 706-713.

77. Augood C, Chakravarthy U, Young I, et al. (2008) Oily fish consumption, dietary docosahexaenoic acid and eicosapentaenoic acid intakes, and associations with neovascular agerelated macular degeneration. Am J Clin Nutr 88, 398-406.

78. Mori TA, Bao DQ, Burke V, et al. (1999) Dietary fish as a major component of a weight-loss diet: effect on serum lipids, glucose, and insulin metabolism in overweight hypertensive subjects. Am J Clin Nutr 70, 817-825.

79. Gunnarsdottir I, Tomasson H, Kiely M, et al. (2008) Inclusion of fish or fish oil in weight-loss diets for young adults: effects on blood lipids. Int $J$ Obes (Lond) 32, 1105-1112.

80. Parra D, Ramel A, Bandarra N, et al. (2008) A diet rich in long chain omega-3 fatty acids modulates satiety in overweight and obese volunteers during weight loss. Appetite 51, 676-680.

81. Kratz M, Callahan HS, Yang PY, et al. (2009) Dietary n-3polyunsaturated fatty acids and energy balance in overweight or moderately obese men and women: a randomized controlled trial. Nutr Metabol 6, 7.

82. Rhodes LE, Durham BH, Fraser WD, et al. (1995) Dietary fish oil reduces basal and ultraviolet B-generated PGE2 levels in skin and increases the threshold to provocation of polymorphic light eruption. J Invest Dermatol 105, $532-535$.

83. Thusgaard M, Christensen JH, Mørn B, et al. (2009) Effect of fish oil ( $n-3$ polyunsaturated fatty acids) on plasma lipids, lipoproteins and inflammatory markers in HIV-infected patients treated with antiretroviral therapy: a randomized, double-blind, placebo-controlled study. Scand J Infect Dis 41, 760-766.

84. Engeset D, Alsaker E, Lund E, et al. (2006) Fish consumption and breast cancer risk. The European Prospective Investigation into Cancer and Nutrition (EPIC). Int $J$ Cancer 119, 175-182.

85. van der Meij BS, Langius JA, Smit EF, et al. (2010) Oral nutritional supplements containing ( $n-3)$ polyunsaturated fatty acids affect the nutritional status of patients with stage III non-small cell lung cancer during multimodality treatment. J Nutr 140, 1774-1780.

86. Cerchietti LC, Navigante AH \& Castro MA (2007) Effects of eicosapentaenoic and docosahexaenoic n-3 fatty acids from fish oil and preferential Cox-2 inhibition on systemic syndromes in patients with advanced lung cancer. Nutr Cancer 59, 14-20.

87. Barber MD, Ross JA, Voss AC, et al. (1999) The effect of an oral nutritional supplement enriched with fish oil on weight-loss in patients with pancreatic cancer. $\mathrm{Br} J$ Cancer 81, 80-86.

88. Leitzmann MF, Stampfer MJ, Michaud DS, et al. (2004) Dietary intake of n-3 and n-6 fatty acids and the risk of prostate cancer. Am J Clin Nutr 80, 204-216.

89. Mozaffarian D (2006) Fish Intake, Contaminants, and Human Health: Evaluating the Risks and the Benefits Part 2 - Health Risks and Optimal Intakes. Cardiol Rounds 10, 1-6.

90. U.S. Environmental Protection AgencyMercury Study Report to Congress Available: http://www.epa.gov/mercury/ report.htm (accessed May 2011)

91. Jeejeebhoy KN (2008) Benefits and risks of a fish diet should we be eating more or less? Nature Clin Pract 5, 178-179.

92. Harris HH, Pickering IJ \& George GN (2003) The chemical form of mercury in fish. Science 302, 1203.

93. Committee on the Toxicological Effects of Methylmercury (2000) Board on Environmental Studies and Toxicology; Commission on Life Sciences; National Research Council. Toxicological Effects of Methylmercury. Washington, DC: National Academy Press.

94. Gochfeld M (2003) Cases of mercury exposure, bioavailability, and absorption. Ecotoxicol Environ Saf 56, 174-179.

95. U.S. EPA (U.S. Environmental Protection Agency) (2001) Methylmercury (MeHg) (CASRN 22967-92-6). Available: http://www.epa.gov/iris/subst/0073.htm (accessed May 2011)

96. Ginsberg GL \& Toal BF (2009) Quantitative approach for incorporating methylmercury risks and omega-3 fatty acid benefits in developing species-specific fish consumption advice. Environ Health Persp 117, 267-275.

97. McDowell MA, Dillon CF, Osterloh J, et al. (2004) Hair mercury levels in U.S. children and women of childbearing age: reference range data from NHANES 1999-2000. Environ Health Perspect 112, 1165-1171. 
98. Grandjean P, Weihe P, White RF, et al. (1997) Cognitive deficit in 7-year-old children with prenatal exposure to methylmercury. Neurotoxicol Teratol 19, 417-428.

99. Grandjean P, Weihe P, White RF \& Debes F (1998) Cognitive performance of children prenatally exposed to "safe" levels of methylmercury. Environ Res 77, 165-172.

100. Kjellstrom T (1989) Physical and mental development of children with prenatal exposure to mercury from fish. Stage II: interviews and psychological tests at age 6 . Stockholm, Sweden: National Swedish Environmental Protection Board; Report 3642. Solna, Sweden: National Swedish Environmental Protection Board.

101. Crump KS, Kjellstrom T, Shipp AM, et al. (1998) Influence of prenatal mercury exposure upon scholastic and psychological test performance: benchmark analysis of a New Zealand cohort. Risk Anal 18, 701-713.

102. Jedrychowski W, Jankowski J, Flak E, et al. (2006) Effects of prenatal exposure to mercury on cognitive and psychomotor function in one-year-old infants: Epidemiologic Cohort Study in Poland. Ann Epidemiol 16, 439-447.

103. Davidson PW, Palumbo D, Myers GJ, et al. (2000) Neurodevelopmental outcomes of Seychellois children from the pilot cohort at 108 months following prenatal exposure to methylmercury from a maternal fish diet. Environ Res 84, $1-11$.

104. Palumbo DR, Cox C, Davidson PW, et al. (2000) Association between prenatal exposure to methylmercury and cognitive functioning in Seychellois children: a reanalysis of the McCarthy Scales of Children's Ability from the main cohort study. Environ Res 84, 81-88.

105. Oken E, Wright RO, Kleinman KP, et al. (2005) Maternal fish consumption, hair mercury, and infant cognition in a U.S. Cohort. Environ Health Perspect 113, 1376-1380.

106. Daniels JL, Longnecker MP, Rowland AS, et al. (2004) Fish intake during pregnancy and early cognitive development of offspring. Epidemiology 15, 394-402.

107. FDA (Food and Drug Administration) (2004) Press Release: FDA and EPA Announce the Revised Consumer Advisory on Methylmercury in Fish, March 19, 2004. Available: http://www.fda.gov/bbs/topics/news/2004/NEW01038.html (accessed May 2011)

108. FDA (2005) Letter Regarding Eggs with Enhanced Omega-3 Fatty Acid Content and a Balanced Ratio of Omega-3/ Omega- 6 Fatty Acids and Reduced Risk of Heart Disease and Sudden Fatal Heart Attack (Docket No. 2004Q-0072). Available: http://www.cfsan.fda.gov/ dms/qhceggs.html (accessed May 2011)

109. FDA (2006) Mercury Levels in Commercial Fish and Shellfish. Available: http://www.cfsan.fda.gov/ frf/sea-mehg. html (accessed May 2011).

110. USDA (2005) Addendum A: EPA and DHA Content of Fish Species. Available: http://www.health.gov/dietaryguidelines/ dga2005/report/HTML/table_g2_adda2.htm (accessed May 2011).

111. U.S. EPA (U.S. Environmental Protection Agency) (1995) IRIS file for Mercuric Chloride. Available: http://www.epa. gov/ncea/iris/subst/0692.htm (accessed May 2011).

112. U.S. EPA (U.S. Environmental Protection Agency) (2004) What You Need to Know about Mercury in Fish and Shellfish. Available: http://www.epa.gov/waterscience/fishadvice/ advice.html (accessed May 2011).

113. Rice DC (2004) The US EPA reference dose for methylmercury: sources of uncertainty. Environ Res 95, 406-413.

114. Rissanen T, Voutilainen S, Nyyssonen K, et al. (2000) Fish oil-derived fatty acids, docosahexaenoic acid and docosapentaenoic acid, and the risk of acute coronary events: the Kuopio ischaemic heart disease risk factor study. Circulation 102, 2677-2679.

115. Guallar E, Sanz-Gallardo MI, van't Veer P, et al. (2002) Mercury, fish oils, and the risk of myocardial infarction. $N$ Engl J Med 347, 1747-1754.

116. Virtanen JK, Voutilainen S, Rissanen TH, et al. (2005) Mercury, fish oils, and risk of acute coronary events and cardiovascular disease, coronary heart disease, and allcause mortality in men in eastern Finland. Arterioscler Thromb Vasc Biol 25, 228-233.

117. Risher JF, Murray HE \& Prince GR (2002) Organic mercury compounds: human exposure and its relevance to public health. Toxicol Ind Health 18, 109-160.

118. He K, Song Y, Daviglus ML, et al. (2004) Fish consumption and incidence of stroke: a meta-analysis of cohort studies. Stroke 35, 1538-1542.

119. Morris MC, Evans DA, Tangney CC, et al. (2005) Fish consumption and cognitive decline with age in a large community study. Arch Neurol 62, 1849-1853.

120. Peet M \& Stokes C (2005) Omega-3 fatty acids in the treatment of psychiatric disorders. Drugs 65, 1051-1059.

121. Young G \& Conquer J (2005) Omega-3 fatty acids and neuropsychiatric disorders. Reprod Nutr Dev 45, 1-28.

122. Mozaffarian D \& Rimm EB (2006) Fish intake, contaminants, and human health evaluating the risks and the benefits. JAMA 296, 1885-1899.

123. Kaul N, Kreml R, Austria JA, et al. (2008) A comparison of fish oil, flaxseed oil and hempseed oil supplementation on selected parameters of cardiovascular health in healthy volunteers. J Am Coll Nutr 27, 51-58.

124. Egert S, Kannenberg F, Somoza V, et al. (2009) Dietary alpha-linolenic acid, EPA, and DHA have differential effects on LDL fatty acid composition but similar effects on serum lipid profiles in normolipidemic humans. J Nutr 139, 861-868.

125. Barceló-Coblijn G, Murphy EJ, Othman R, et al. (2008) Flaxseed oil and fish-oil capsule consumption alters human red blood cell n-3 fatty acid composition: a multiple-dosing trial comparing 2 sources of n-3 fatty acid. Am J Clin Nutr 88, 801-809.

126. Sioen I, Hacquebard M, Hick G, et al. (2009) Effect of ALA-enriched food supply on cardiovascular risk factors in males. Lipids 44, 603-611.

127. Bloedon LT, Balikai S, Chittams J, et al. (2008) Flaxseed and cardiovascular risk factors: results from a double blind, randomized, controlled clinical trial. J Am Coll Nutr 27, 65-74.

128. Dodin S, Cunnane SC, Mâsse B, et al. (2008) Flaxseed on cardiovascular disease markers in healthy menopausal women: a randomized, double-blind, placebo-controlled trial. Nutrition 24, 23-30.

129. Campos H, Baylin A \& Willett WC (2008) Alpha-linolenic acid and risk of nonfatal acute myocardial infarction. Circulation 118, 339-345.

130. Yamagishi K, Nettleton JA, Folsom AR \& ARIC Study Investigators (2008) Plasma fatty acid composition and incident heart failure in middle-aged adults: the Atherosclerosis Risk in Communities (ARIC) Study. Am Heart $J$ 156, 965-974.

131. Warensjö E, Sundström J, Vessby B, et al. (2008) Markers of dietary fat quality and fatty acid desaturation as predictors of total and cardiovascular mortality: a population-based prospective study. Am J Clin Nutr 88, 203-209.

132. Park Y, Park S, Yi H, et al. (2009) Low level of n-3 polyunsaturated fatty acids in erythrocytes is a risk factor for 
both acute ischemic and hemorrhagic stroke in Koreans. Nutr Res 29, 825-830.

133. Virtanen JK, Mursu J, Voutilainen S, et al. (2009) Serum long-chain n-3 polyunsaturated fatty acids and risk of hospital diagnosis of atrial fibrillation in men. Circulation 120, $2315-2321$

134. Lemaitre RN, King IB, Sotoodehnia N, et al. (2009) Red blood cell membrane alpha-linolenic acid and the risk of sudden cardiac arrest. Metabolism 58, 534-540.

135. Finnegan YE, Minihane AM, Leigh-Firbank EC, et al. (2003) Plant- and marine-derived n-3 polyunsaturated fatty acids have differential effects on fasting and postprandial blood lipid concentrations and on the susceptibility of LDL to oxidative modification in moderately hyperlipidemic subjects. Am J Clin Nutr 77, 783-795.

136. Malgeunsinae Kim, Jung Hyun Nam, Dong Hoon Oh, et al. (2010) Erythrocyte alpha-linolenic acid is associated with the risk for mild dementia in Korean elderly. Nutr Res 30, 756-761.

137. Griel AE, Kris-Etherton PM, Hilpert KF, et al. (2007) An increase in dietary n-3 fatty acids decreases a marker of bone resorption in humans. Nutr J 6, 2.

138. Taylor CG, Noto AD, Stringer DM, et al. (2010) Dietary milled flaxseed and flaxseed oil improve $N$-3 fatty acid status and do not affect glycemic control in individuals with well-controlled type 2 diabetes. J Am Coll Nutr 29, 72-80.

139. Paschos GK, Magkos F, Panagiotakos DB, et al. (2007) Dietary supplementation with flaxseed oil lowers blood pressure in dyslipidaemic patients. Eur J Clin Nutr 61, 1201-1206.

140. Paschos GK, Zampelas A, Panagiotakos DB, et al. (2007) Effects of flaxseed oil supplementation on plasma adiponectin levels in dyslipidemic men. Eur J Clin Nutr 46, 315-320.

141. Harper CR, Edwards MC \& Jacobson TA (2007) Flaxseed oil supplementation does not affect plasma lipoprotein concentration or particle size in human subjects. $J$ Nutr 136, $2844-2848$.

142. Cunnane SC, Hamadeh MJ, Liede AC, et al. (1995) Nutritional attributes of traditional flaxseed in healthy young adults. Am J Clin Nutr 61, 62-68.

143. Kew S, Banerjee T, Minihane AM, et al. (2003) Lack of effect of foods enriched with plant- or marine-derived n-3 fatty acids on human immune function. Am J Clin Nutr 77, 1287-1295.

144. Hoffman D, Ziegler E, Mitmesser SH, et al. (2008) Soybased infant formula supplemented with DHA and ARA supports growth and increases circulating levels of these fatty acids in infants. Lipids 43, 29-35.

145. Egert S, Somoza V, Kannenberg F, et al. (2007) Influence of three rapeseed oil-rich diets, fortified with alpha-linolenic acid, eicosapentaenoic acid or docosahexaenoic acid on the composition and oxidizability of low-density lipoproteins: results of a controlled study in healthy volunteers. Eur J Clin Nutr 61, 314-325.

146. Visioli F, Rise P, Plasmati E, et al. (2000) Very low intakes of $n-3$ fatty acids incorporated into bovine milk reduce plasma triacylglycerols and increase HDL-cholesterol concentrations in healthy subjects. Pharmacol Res 41, $571-576$.

147. Baró L, Fonollá J, Peña JL, et al. (2003) n-3 fatty acids plus oleic acid supplemented milk reduces total and LDL cholesterol, homocysteine and levels of endothelial adhesion molecules in healthy humans. Clin Nutr 22, 175-182.
148. Carrero JJ, Baró L, Fonollá J, et al. (2004) Cardiovascular effects of milk enriched with n-3 polyunsaturated fatty acids, oleic acid folic acid and vitamins E, B6 and B12 in volunteers with mild hyperlipidaemia. Nutrition 20, 521-527.

149. Dawczynski C, Martin L, Wagner A, et al. (2010) n-3 LCPUFAs-enriched dairy products are able to reduce cardiovascular risk factors: a double-blind, cross-over study. Clin Nutr 29, 592-599.

150. Chong MF, Lockyer S, Saunders CJ, et al. (2010) Long chain n-3 PUFA-rich meal reduced postprandial measures of arterial stiffness. Clin Nutr 29, 678-681.

151. Estévez-González MD, Saavedra-Santana P \& BetancorLeón P (1998) Reduction of serum cholesterol and low-density lipoprotein cholesterol levels in a juvenile population after isocaloric substitution of whole milk with a milk preparation (skimmed milk enriched with oleic acid). J Pediatr 132, 85-89.

152. Cobiac L, Clifton PM, Abbey M, et al. (1991) Lipid, lipoprotein, and hemostatic effects of fish vs fish-oil n-3 fatty acids in mildly hyperlipidemic males. Am J Clin Nutr 53, 1210-1216.

153. Fonollá J, López-Huertas E, Machado FJ, et al. (2009) Milk enriched with "healthy fatty acids" improves cardiovascular risk markers and nutritional status in human volunteers. Nutrition 25, 408-414.

154. Carrero JJ, López-Huertas E, Salmerón LM, et al. (2005) Daily supplementation with (n-3) PUFAs, oleic acid, folic acid, and vitamins B- 6 and $\mathrm{E}$ increases pain free walking distance and improves risk factors in men with peripheral vascular disease. J Nutr 135, 1393-1399.

155. Carrero JJ, Fonollá J, Marti JL, et al. (2007) Intake of fish oil, oleic acid, folic acid, and vitamins B-6 and E for 1 year decreases plasma $\mathrm{C}$ reactive protein and reduces coronary heart disease risk factors in male patients in a cardiac rehabilitation program. J Nutr 137, 384-390.

156. Benito P, Caballero J, Moreno J, et al. (2006) Effects of milk enriched with omega-3 fatty acid, oleic acid and folic acid in patients with metabolic syndrome. Clin Nutr 25, 581-587.

157. Givens DI \& Gibbs RA (2008) Current intakes of EPA and DHA in European populations and the potential of animal-derived foods to increase them. Proc Nutr Soc 67, 273-280.

158. Kronberg SL, Barcelo-Coblijn G, Shin J, et al. (2006) Bovine muscle $n-3$ fatty acid content is increased with flaxseed feeding. Lipids 41, 1059-1068.

159. Kjos NP, Skrede A \& Overland M (1999) Effects of dietary fish silage and fish fat on growth performance and sensory quality of growing-finishing pigs. Can J Anim Sci 79, 139-147.

160. Jaturasitha S, Khiaosa-ard R, Pongpiachan P, et al. (2009) Early deposition of $n-3$ fatty acids from tuna oil in lean and adipose tissue of fattening pigs is mainly permanent. J Anim Sci 87, 693-703.

161. Muench S \& Watzl B (2010) Incorporation of ingredients rich in omega-3 fatty acids into functional meat products. Mitteil Fleischf Kulmb 49, 39-48.

162. Cachaldora P, Garcia-Rebollar P \& Alvarez C (2009) Double enrichment of chicken eggs with conjugated linoleic acid and $\mathrm{n}-3$ fatty acids through dietary fat supplementation. Animal Feed Sci Tech 144, 315-326.

163. Bovet P, Faeh D, Madeleine G, et al. (2007) Decrease in blood triglycerides associated with the consumption of eggs of hens fed with food supplemented with fish oil. Nutr Metab Cardiovasc Dis 17, 280-287.

164. Rizzi L, Bochicchio D \& Bargellini A (2009) Effects of dietary microalgae, other lipid sources, inorganic selenium 
and iodine on yolk n-3 fatty acid composition, selenium content and quality of eggs in laying hens. J Sci Food Agric 89, 1775-1781.

165. Bourre JM (2005) Where to find omega-3 fatty acids and how feeding animals with diet enriched in omega- 3 fatty acids to increase nutritional value of derived products for human: what is actually useful? J Nutr Health Aging $\mathbf{9}$, 232-242.

166. Tou JC, Jaczynski J \& Chen YC (2007) Krill for human consumption: nutritional value and potential health benefits. Nutr Rev 65, 63-77.

167. Maki KC, Reeves MS, Farmer M, et al. (2009) Krill oil supplementation increases plasma concentrations of eicosapentaenoic and docosahexaenoic acids in overweight and obese men and women. Nutr Res 29, 609-615.
168. Bunea R, El Farrah K \& Deutsch L (2004) Evaluation of the effects of Neptune Krill Oil on the clinical course of hyperlipidemia. Altern Med Rev 9, 420-428.

169. Sampalis F, Bunea R, Pelland MF, et al. (2003) Evaluation of the effects of Neptune Krill Oil on the management of premenstrual syndrome and dysmenorrheal. Altern Med Rev 8, 171-179.

170. Conquer JA, Cheryk LA, Chan E, et al. (1999) Effect of supplementation with dietary seal oil on selected cardiovascular risk factors and hemostatic variables in healthy male subjects. Thromb Res 96, 239-250.

171. Zhu FS, Liu S, Chen XM, et al. (2008) Effects of n-3 polyunsaturated fatty acids from seal oils on nonalcoholic fatty liver disease associated with hyperlipidemia. World $J$ Gastroenterol 14, 6395-6400. 